Helsinki University of TeChNOLOGY

Department of Electrical and Communications Engineering

Networking Laboratory

Elena Sirén

\title{
Optical Packet Switching
}

Thesis submitted in partial fulfillment of the requirements for the degree of Master of Scince in Engineering

Espoo, Finland, March 26, 2002

Supervisor: Professor Jorma Virtamo, HUT

Instructor: Esa Hyytiä, Lic.Sc. (Tech.), HUT 
Author:

Elena Sirén

Title of the thesis: Optical Packet Switching

Date:

March 26

Number of pages: 76

Faculty: $\quad$ Electrical and Communications Engineering

Laboratory: S-38 Networking Technology

Supervisor: Professor Jorma Virtamo

Instructor: Esa Hyytiä, Lic.Sc. (Tech.)

In this thesis recent research in the area of optical packet switching is reviewed, the main problems in the area are identified and a possible solution to some of the problems, optical burst switching, is studied in more detail. Optical burst switching and the JET protocol are studied by means of a simulation program specially built for this purpose.

Optical packet networks are networks, where the switching operations are performed optically, and the data remains in optical domain through the transmission. There have already been several implementations of optical packet switching in laboratory environment, but commercial solutions are not expected within a few next years. This is due to the infancy of optical devices. Although the networks and the components have developed rapidly, there are still many unsolved questions. The main problems at the moment are the lack of optical random access memory and very high switching rates needed. A few solutions that try to overcome these problems, and still offer the benefits of optical packet switching, have been proposed. One interesting alternative is optical burst switching.

In the empirical part of the thesis the use of the JET protocol in optical burst switching network was studied. In contrast to earlier research, in this work the performance of JET protocol was studied in networks without wavelength conversion. As a result it was found that with relatively small number of buffers a performance that is comparable to that of a wavelength converted version can be achieved.

Keywords: optical packet swiching, optical burst switching, JET protocol 


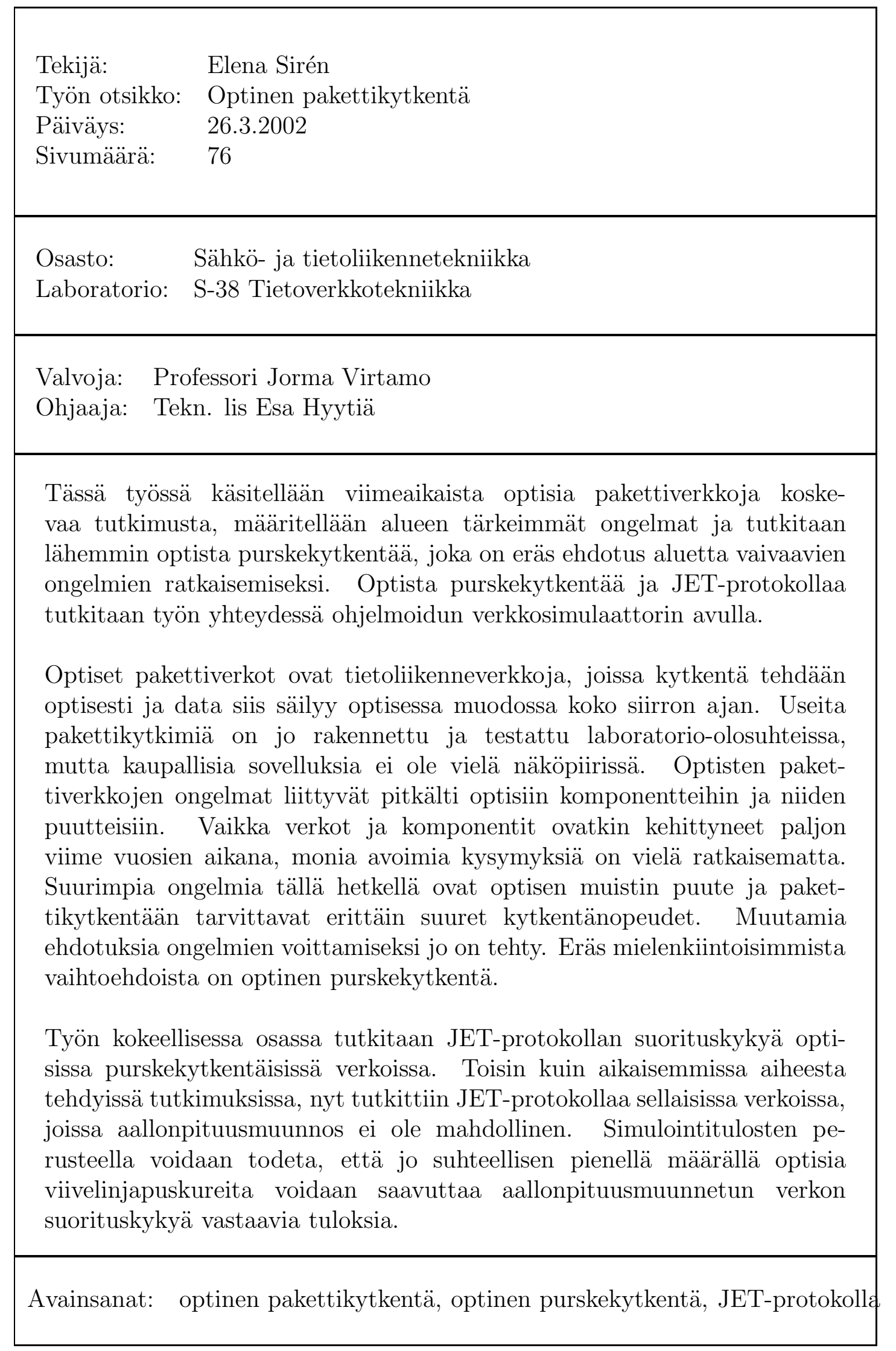




\section{Preface}

This work was written in the Networking Laboratory at the Helsinki University of Technology as a part of Optical Access Networks (OAN) project.

I would like to thank all the people who have helped me during this work. Especially I would like to express gratitude to the supervisor of the thesis, professor Jorma Virtamo, for his guidance during the work and to my instructor, Esa Hyytiä, Lic.Sc. (Tech), for his valuable help. My parents deserve special thanks for the support and encouragement I have got from them during my studies. Finally, I would also like to thank Klaus, because without him it would have been so much harder to get this thesis into covers.

Espoo, 23.3.2002.

Elena Sirén 


\section{Contents}

Acronyms ............................. ix

1 Introduction $\quad 1$

1.1 Background and Motivation of the Thesis . . . . . . . . . . . 1

1.2 Structure of the Thesis . . . . . . . . . . . . . . 1

1.3 Goal of the Thesis . . . . . . . . . . . . . . . . 2

2 Introduction to Optical Networks $\quad 3$

2.1 Multiplexing Techniques ................. 3

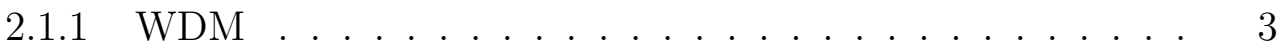

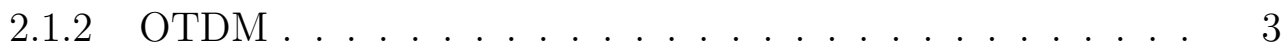

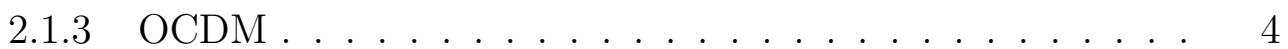

2.2 SONET/SDH . . . . . . . . . . . . . . . 4

2.3 Broadcast-and-Select Networks . . . . . . . . . . . . . 5

2.3.1 Multihop Networks . . . . . . . . . . . . . . 5

2.3.2 Single-Hop Networks . . . . . . . . . . . . . . 5

2.4 Wavelength-Routed Networks . . . . . . . . . . . . . 5

2.5 Optical Packet Switching Networks . . . . . . . . . . . 6

2.6 Evolution of Optical Networks . . . . . . . . . . . . . 6

2.6.1 Evolution of Network Topologies . . . . . . . . . . . 6

2.6.2 Evolution of Transmission Capacity . . . . . . . . . . . . 7

2.6.3 Evolution of Networks . . . . . . . . . . . . . 7

3 Optical Components $\quad 8$

3.1 Passive Optical Components . . . . . . . . . . . . . . 8

3.1 .1 Fiber . . . . . . . . . . . . . . . . . . 8

3.1 .2 Couplers . . . . . . . . . . . . . . . . . . . . . . . 9

3.1 .3 Isolators and Circulators . . . . . . . . . . . . . . . . 9

3.1.4 Multiplexers and Filters . . . . . . . . . . . . . . 10

3.2 Active Optical Components . . . . . . . . . . . . . . . . . . 13

3.2.1 Amplifiers . . . . . . . . . . . . . . . . . . . 13

3.2.2 Transmitters and Receivers . . . . . . . . . . . . . . 15

3.2.3 Wavelength Converters . . . . . . . . . . . . . . 16

4 Switching 18

4.1 Introduction to Switching . . . . . . . . . . . . . . . . . 18

4.1.1 Space Switch and Wavelength Router . . . . . . . . . . 18

4.1.2 Classification of Switches . . . . . . . . . . . . 20

4.2 Packet Switch Architectures . . . . . . . . . . . . . . 21 
4.2.1 Space-Switching-Based Switches . . . . . . . . . . . . . . 22

4.2.2 Wavelength Routing Switches . . . . . . . . . . . 25

4.2.3 Broadcast-and-Select Switches . . . . . . . . . . . . . . . 31

4.2.4 Switches in Slotted Networks . . . . . . . . . . . . . 34

4.2.5 Comparison and Conclusions . . . . . . . . . . . . . . 37

4.3 Contention Resolution . . . . . . . . . . . . . . . . . . 38

4.3.1 Space Deflection . . . . . . . . . . . . . . . . . . 38

4.3 .2 Optical Buffering . . . . . . . . . . . . . . . . . . . . . . . . 39

4.3.3 Wavelength Conversion . . . . . . . . . . . . . . 39

4.3.4 Performance of Different Contention Resolution Schemes . 40

5 Recent Research Projects in Optical Packet Switching 41

5.1 ATMOS . . . . . . . . . . . . . . . . . 41

$5.2 \mathrm{KEOPS} \ldots \ldots \ldots \ldots \ldots \ldots \ldots$

5.2 .1 Components . . . . . . . . . . . . . . . . . . . . . . . . . . . 42

5.2 .2 Switch Architectures . . . . . . . . . . . . . 43

5.2 .3 Packet Format . . . . . . . . . . . . . . . . . . 44

5.3 WASPNET . . . . . . . . . . . . . . . . 45

5.3 .1 The WASPNET Switch . . . . . . . . . . . . 46

5.3 .2 Network Control . . . . . . . . . . . . . . . . . . . . 47

5.3 .3 Packet Format . . . . . . . . . . . . . . . . . . . . . . 48

5.4 FLAMINGO . . . . . . . . . . . . . . . . . . . . . . . 48

5.5 DAVID . . . . . . . . . . . . . . . . . . . . . . . 49

5.6 Other Projects . . . . . . . . . . . . . . 50

6 Strong and Weak Points of Optical Packet Switching 51

6.1 Unsolved Questions . . . . . . . . . . . . . . . . 51

6.1 Header Processing . . . . . . . . . . . . . . 51

6.1.2 Synchronization . . . . . . . . . . . . . . . . 52

6.1.3 Regeneration of Optical Signals . . . . . . . . . . . . . 52

6.1 .4 IP over Optical Networks . . . . . . . . . . . . . . . . . . . . 52

6.2 Alternatives and Comparison . . . . . . . . . . . . . . . 52

6.2.1 Optical versus Electronic Networks . . . . . . . . . . 52

6.2.2 Optical Versus Electro-Optical Devices . . . . . . . . . 53

6.2.3 Packet Versus Circuit Switching in Optical Networks . . . 53

6.2.4 Burst Switching Versus Traditional

Packet Switching . . . . . . . . . . . . . . . 54

6.3 The Future of Optical Packet Networks . . . . . . . . . . . . . 54

7 Simulations of the JET protocol in Burst Switching Network 56

7.1 JET Protocol . . . . . . . . . . . . . . . . . . 56

7.2 Network Model . . . . . . . . . . . . . . . . . . . . 57

7.3 Simulation Program . . . . . . . . . . . . . . . . . 58

7.4 Small Example Network . . . . . . . . . . . . . . . . . . . 61

7.4.1 Different Delay Line Configurations . . . . . . . . . . . 68

7.5 Efficiency in Larger Network . . . . . . . . . . . . . . . . . 71

7.6 Discussion . . . . . . . . . . . . . . . . . . 73 
8 Conclusions 


\section{Acronyms}

- AWG Arrayed Waveguide Grating is a component that is used as a multiplexer or as a demultiplexer in optical devices.

- AWGM Arrayed Waveguide Grating Multiplexer, a device that consists of an AWG and two star couplers. Switches signals to an output according to the input port and wavelength.

- DFB Distributed Feedback Laser, a narrow band single mode laser.

- EDFA Erbium-Doped Fiber Amplifier is an all-optical amplifier suitable for long-haul transmission.

- JET Just-Enough-Time, a protocol that is used for resource allocation in optical burst switching networks.

- LED Light Emitting Diode, a cheap and inefficient device that is used as a light source.

- OCDM Optical Code Division Multiplexing, transmission of signals on the same fiber on s common wavelength band with different codes.

- OTDM Optical Time Division Multiplexing, see TDM.

- OXC Optical Crossconnect, an optical circuit switch.

- PDH Plesiochronous Digital Hierarchy, a digital transmission standard that was used before SDH/SONET.

- SOA Semiconductor Optical Amplifier, a basic component of optical devices. SOA gates are used, e.g., in optical space switches.

- SONET/SDH A digital transmission standard. Higher layers use optical packet switching, optical layer is point to point.

- TDM Time Division Multiplexing, compressing several signals to the same fiber by increasing the data rate.

- TWC Tunable Wavelength Converter, a device that converts signals from one wavelength to another. Tunable wavelength converters can handle several input wavelengths and can change also the output wavelength.

- WDM Wavelength Division Multiplexing, transmitting several signals in the same fiber with different wavelengths. 


\section{Chapter 1}

\section{Introduction}

\subsection{Background and Motivation of the Thesis}

Optical packet networks are networks, where the switching operations are performed optically. Since the first optical networks, the components and the networks themselves have developed rapidly. However, there are still many unsolved questions. At the moment many networks are hybrid, i.e. the network itself and a part of devices are optical, but electronics are used also. All-optical circuit switched networks become more common all the time, but the all-optical packet networks are still in the infancy, though developing fast. The main problem is the infancy of optical devices, expecially the lack of optical random access memory.

In this work the latest research concerning optical packet networks is reviewed. The transfer rates are growing rapidly. On the other hand the amount of transferred data is growing faster than the amount of transferred voice. Therefore the significance of packet switched networks is increasing and at the same time the need for a more efficient data transfer medium has also grown. Although optical packet networks will not have commercial significance until a couple of years at the earliest, in the long run they are an attractive alternative.

\subsection{Structure of the Thesis}

In the first section of Chapter 2, the multiplexing techniques are introduced. Then the history and the evolution of optical networks from the beginning to today are generally discussed, together with different network types. In Chapter 3, the basic components of optical networks are introduced and then, in Chapter 4 , the optical packet switches are studied more carefully. The focus is on switches, but the switching operation and contention resolution are also briefly covered. In Chapter 5 research projects dealing with optical packet networks are discussed. The results of the research considering switching and switching fabrics are already partly addressed in Chapter 4 , but now the research projects are presented in more detail. In Chapter 6 optical packet switching is compared with other alternatives, and its strong and weak points are identified. Also, the reader is 
given a wider picture of the area, and the significance of optical packet switching in the world of telecommunication is discussed. In Chapter 7 the simulations of the JET protocol in optical burst switching network is discussed. The simulator program is described and the empirical results obtained are presented and analyzed. Chapter 8 contains the conclusions.

\subsection{Goal of the Thesis}

This thesis is a survey of the state-of-art of optical packet networks. The recent research done on the area of optical packet switching is discussed and the relevant traffic problems are identified. The second objective of this work is to study optical burst switching and the JET protocol by means of a simulation program specially built for this purpose in this thesis. 


\section{Chapter 2}

\section{Introduction to Optical Networks}

\subsection{Multiplexing Techniques}

\subsubsection{WDM}

WDM is an optical version for FDM. The idea is that several signals are transmitted at the same time in the same fiber at different wavelengths. This way WDM provides many virtual fibers on a single physical fiber. Today WDM is the most popular alternative to multiplex signals in the optical domain. WDM is also the most mature all-optical multiplexing technique. Its main advantages are the signal transparency, scalability and flexibility; the existing fiber lines can be upgraded by implementing WDM. The main problems are need for flat gain amplifiers, increasing noise when the number of channels increases, and the limits in channel spacing and in the number of channels caused by the wave mixing and the cross-phase modulation.

The key parameters of any multiplexing system are the total capacity of the system, number of channels, the spectral efficiency and the transmission distance. WDM systems capable of multiplexing up to $40 \times 10 \mathrm{Gbit} / \mathrm{s}$ channels in a single fiber have been in commercial use a couple of years $\left[\mathrm{LDA}^{+} 99\right]$. In the laboratory environment systems with capacity over $1 \mathrm{Tbit} / \mathrm{s}$ per link have been implemented [KT01], e.g., in Japan, a system capable of transmitting $100 \times 20$ Gbit/s over 2700 $\mathrm{km}$ distance and another system capable of transmitting $100 \times 10 \mathrm{Gbit} / \mathrm{s}$ over 8000 $\mathrm{km}$ distance have been implemented.

\subsubsection{OTDM}

OTDM is a promising multiplexing technique, but it is still on research level. It is a very similar to electronic TDM, the only difference is that OTDM is faster, and the devices used in OTDM networks are optical. The operational principle of both TDM and OTDM is to interleave several signals in time and then direct them to the same fiber.

The main advantages of this multiplexing technique are the facts that only 
one source is required and the node equipment is simpler in the single channel architecture. The problems concerning OTDM are associated with the immaturity of all-optical devices. Very fast lasers are needed and timing synchronization and alignment are still problems. Additionally, although a system were technically implementable, it would not be economically viable. Another weakness is that the OTDM is not a transparent multiplexing technique. However, in the future OTDM will probably be a key technology, though at least in the beginning only when combined with WDM [LDA+99].

\subsubsection{OCDM}

In Optical Code Division Multiplexing (OCDM) each optical code (channel) is assigned to its own, independent path (OCP). Cells with different OCPs can be transmitted in the same fiber at the same time. OCDM is transparent and no synchronization between different channels is needed [KOS98]. Using OCDM in addition to WDM can remarkably improve the communication capacity of the network. OCDM can be also used to improve the communication security.

\section{$2.2 \mathrm{SONET} / \mathrm{SDH}$}

The initial digital transmission standard was Plesiochronous digital hierarchy $(\mathrm{PDH})$, which had several drawbacks. The versions of PDH in the North America and Europa were different, the multiplexing was complex and there was only limited amount of overhead available for the network management [LDA+99]. These limitations led to an implementation of a new standard. First Synchronous Optical network (SONET) was implemented in North-America in 1984. Soon almost similar network transmission technology, Synchronous Digital Hierarchy (SDH), was in use in the Europe. Today SONET is in use in North-America and SDH in Japan and Europe. Differences between these two technologies are minor, and will not be discussed here.

SONET/SDH is a standard for high-speed, synchronous transmission network. Usually SONET/SDH is a ring or mesh network (see Figure 2.1), where optical fibers are used, but all the routing and switching is done electronically. In other words, SONET/SDH is a point-to-point architecture, where a signal is transmitted in fiber optically, but converted to electronic form in every node. SONET/SDH uses TDM as a multiplexing technique.

One of the advantages SONET/SDH offers compared to PDH is the network management. Additionally it is vendor-independent thanks to the standardization. And although the capacity of SONET/SDH is smaller than the capacity of WDM systems, it is still a high-speed system. SONET/SDH communications support various data rates ranging from $51.84 \mathrm{Mbit} / \mathrm{s}$ up to $10 \mathrm{Gbit} / \mathrm{s}$. SONET/SDH has its drawbacks, but it will be in use for several years from now $\left[\mathrm{LDA}^{+} 99\right]$. Today most of the new systems installed are based on SONET or 
SDH technologies. The use of WDM networks will probably increase fast, but the SONET/SDH will be used simultaneously.

\subsection{Broadcast-and-Select Networks}

The main idea of broadcast-and-select networks is that the data is broadcasted at a special wavelength to all nodes. The receivers accept only certain wavelengths, i.e., data channels. Therefore the data is rejected in those nodes that it does not belong to. Typical shapes for these networks are star and bus topologies [EM00] (see Figure 2.1). Broadcast-and-select networks have been extensively studied lately, and prototypes have been developed [XPR01].

\subsubsection{Multihop Networks}

In multihop networks every node has only a few fixed transmitters and receivers. Therefore, a signal cannot always be directly transmitted from source node to destination node. Instead signals have to be received at some intermediate nodes along the way, converted to electronic form and retransmitted.

\subsubsection{Single-Hop Networks}

In single-hop networks data is converted to electrical form only at the end of the path. These networks can be either circuit or packet switched, and packet switched networks furthermore connectionless or connection oriented. Single-hop networks can function as packet networks if fast tunable receivers and/or transmitters are used. Actually, the first implemented optical packet networks were single-hop broadcast-and-select networks.

\subsection{Wavelength-Routed Networks}

Wavelength-routed networks currently represent the most promising technology for optical backbone networks [XPR01]. They can be either single-hop or multihop. The network topology is typically a mesh. In these networks the routing and switching functions are done on a lower layer called the optical layer. The data transfer is done using light paths. Then network is transparent and protocol insensitive and component expenses are saved because less higher layer logic is needed. This network is ideal for circuit switching but not suitable for packet switching. However, hybrid networks, i.e., networks consisting of both circuit and packet switching can be implemented $\left[\mathrm{VKM}^{+} 01\right]$. 


\subsection{Optical Packet Switching Networks}

Today's optical packet networks are not entirely optical. The signals are converted to electrical form before switching and processing. This means that the major advantages of optical packet switching, i.e., speed and efficiency, are lost due to the increased delay. All-optical packet networks are not coming to commercial use in a few next years. It is not entirely sure yet, whether they will be used at all. However, the development is fast, and many demonstrations of optical packet switches and networks have already been conducted in laboratories [CJR ${ }^{+}$98, TZ99, YMD00a]. The biggest problem at the moment is the lack of optical RAM needed for buffering the packets. Additionally, very high switching rates needed in packet networks cause problems. In the near future, the development seems to lead to integration of optical and electronic networks and the use of optical burst switching (see Chapter 6.2.4).

\subsection{Evolution of Optical Networks}

\subsubsection{Evolution of Network Topologies}

Point-to-point architecture is the simplest type of network topology. Packets are transmitted optically between nodes, but opto-electronic conversion is performed in every node. SONET/SDH is one example of such network. A little more advanced alternative is to use bus, ring or star topologies (see Figure 2.1). In WDM networks the ring topology is preferred [ $\left.\mathrm{LDA}^{+} 99\right]$. The mesh topology is more advantageous compared to the previous because it tolerates fiber cuts better, since there are more alternative routes. Additionally, a node with a high traffic rate can be connected to several nodes, and a node with data traffic on a single node can be connected to this single node only. Unfortunately a mesh network is more difficult to implement than the others, because it requires routing and switching. The first WDM networks in the mid 1990's were of the pointto-point type. Then optical add/drop elements were implemented and in the late 1990's the ring topology became more common. Today there already are mesh networks $\left[\mathrm{LDA}^{+} 99\right]$. A part of optical packet networks implemented in the laboratory environment have been mesh networks. Probably the commercial packet networks will follow the same evolution as the WDM networks before.
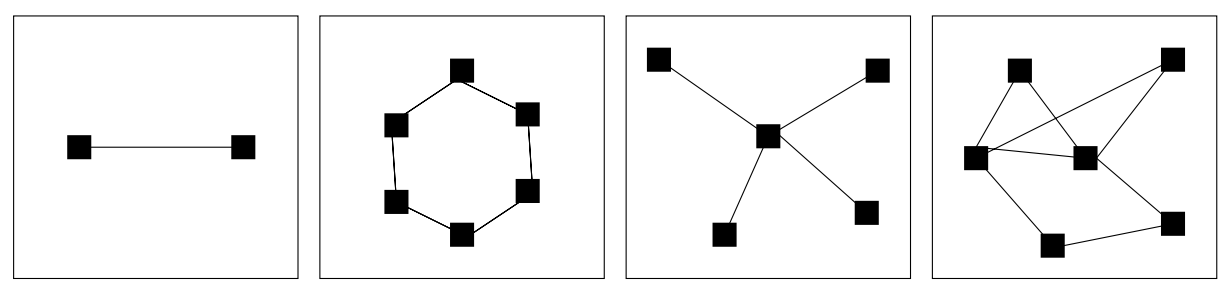

Figure 2.1: The evolution of network topologies: transmission, ring, star and mesh 


\subsubsection{Evolution of Transmission Capacity}

The rate of growth of transmission capacity has accelerated during past few years. In the mid 1990's the increase was 30\% per year, today it is $60 \%$ [ $\mathrm{LDA}^{+} 99$ ]. Table 2.1 describes the predicted growth of the total fiber capacity and the user bit rate.

\begin{tabular}{|c|c|c|c|c|}
\hline \hline & 1995 & 2000 & 2005 & 2010 \\
\hline \hline Total & $20-40 \mathrm{Gbit} / \mathrm{s}$ & $800 \mathrm{Gbit} / \mathrm{s}$ & $\geq 1 \mathrm{Tbit} / \mathrm{s}$ & \\
capacity & & & & \\
\hline User & POTS & ADSL & optical, ADSL & optical, \\
bit rate & $64 \mathrm{kbit} / \mathrm{s}$ & $2-8 \mathrm{Mbit} / \mathrm{s}$ & $155 \mathrm{Mbit} / \mathrm{s} ;$ & $\begin{array}{c}1 \text { ectronic } \\
\end{array}$ \\
& & & $2,10,50 \mathrm{Mbit} / \mathrm{s}$ & $622 \mathrm{Mbit} / \mathrm{s}$, \\
& & & $100 \mathrm{Mbit} / \mathrm{s}$ \\
\hline \hline
\end{tabular}

Table 2.1: The evolution of the transmission capacity. Adopted from [LDA $\left.{ }^{+} 99\right]$

\subsubsection{Evolution of Networks}

The first optical networks were implemented decades ago, but the actual commercial exploitation of optical networks is a relatively new phenomenon, as Figure 2.2 shows. SONET/SDH networks have become common in the last couple of years and WDM networks will have their climax in the latter half of 2000s. Optical packet networks will be in commercial use in the 2010's at the earliest. The increase is still fast if the capacity will increase at $60 \%$ per year as Figure 2.2 suggests.

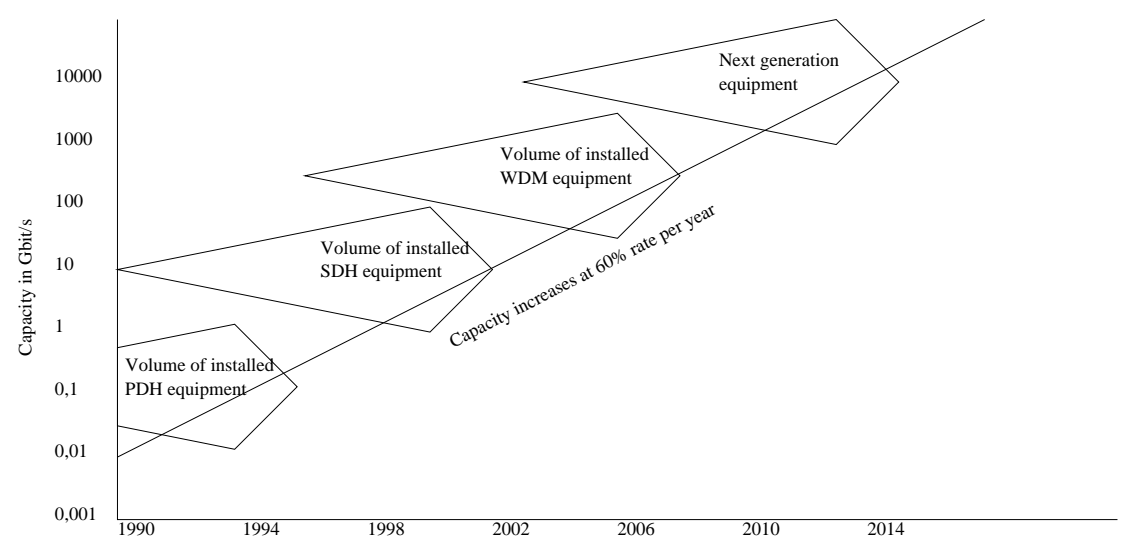

Figure 2.2: The evolution of optical networks. Adopted from $\left[\mathrm{LDA}^{+} 99\right]$. 


\section{Chapter 3}

\section{Optical Components}

\subsection{Passive Optical Components}

\subsubsection{Fiber}

As a transmission medium the fiber has several benefits over the copper. The attenuation is lower, the transfer rates can be higher and there is no electromagnetic interference. Additionally, the fiber is lighter and stronger than the copper. Naturally the fiber also has some weaknesses, such as dispersion, nonlinear refraction or attenuation. Yet, compared to other transmission media the fiber is an attractive alternative. In fact, as early as in the 1980's it was clear that the optical fiber would be necessary to support high capacity systems [LDA ${ }^{+}$99].

An optical fiber consists of a core and a cladding surrounding it. Both are made of pure silica glass, but their refractive indices are different. The use of the optical fiber as a transmission medium is based on this. Depending on the difference between refraction indexes and the angle at which the light strikes the interface of two different transmission media, it either reflects or refracts. If the refraction indexes differ sufficiently, a part of light confronting the interface is reflected. By controlling the angle at which the light waves are transmitted and encounter the interface of core and cladding, the proportion of the reflected light can be increased and the signal can be contained within the core and guided to the other end of the fiber. For further information, see [RS98] and [Red98].

The signal traveling in the fiber has a finite number of possible paths. These paths are called modes. The optical fiber can be either multimode or single-mode. Multimode fibers have an about five times larger core. Because the propagation time along different paths is different, multimode fibers suffer from intermodal dispersion, i.e. differences in the propagation times of waves in the fiber. The multimode fiber is simpler to manufacture making it cheaper, but the single-mode fiber has several advantages. Today multimode fibers are used if a cheap alternative is needed, whereas the single-mode fibers are used over the longer distances.

The most remarkable problem in using optical fiber is the dispersion. The problems resulting from dispersion increase as the bit rates increase, which make 
this problem significant. There are three kinds of dispersion. Chromatic dispersion follows from the fact the color of the light has an effect on its propagation speed. If the core of the single mode fiber is asymmetric, and the light beams traveling along different sides of it have therefore different speeds, the polarization mode dispersion occurs. Slope mismatch dispersion occurs in single-mode fibers, because dispersion varies with wavelength.

\subsubsection{Couplers}

Couplers are simple passive optical components which are used to split or combine signals. A coupler consist of $n$ input and $m$ output ports. A $1 \times n$ coupler is called a splitter and an $n \times 1$ coupler is called a combiner. Figure 3.1 describes a 3 -dB $2 \times 2$ coupler. In $2 \times 2$ coupler a part of input signal 1 is directed to output port 1 and the rest to the output port 2 . In a similar way a part of input signal 2 is guided to both output ports. The fractions directed to output ports can be either non-equal or equal (as in the coupler in Figure 3.1). Couplers function as building blocks of other components. A coupler can also be used for measurements by separating a small fraction of signal for this purpose. For further information of couplers, see [RS98] and [Muk97].

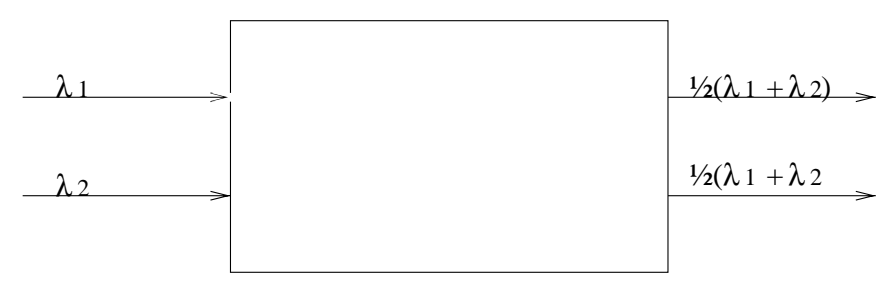

Figure 3.1: A $2 \times 23-\mathrm{dB}$ coupler

\subsubsection{Isolators and Circulators}

Isolators are devices that allow transmission only to one direction and block the transmission to the other direction. This way reflections from, e.g., amplifiers or lasers can be prevented. Typically the insertion loss, i.e. the loss in the forward direction is around $1 \mathrm{~dB}$ and the isolation, i.e. the loss in the reverse direction, is approximately 40 to $50 \mathrm{~dB}$ [RS98]. A circulator is a device similar to an isolator, but with multiple ports. Figure 3.2 describes a circulator with 4 input and 4 output ports. A signal from each port is directed to one output port and blocked in all the other ports, as in Figure 3.2. Circulators can be used as a part of add/drop multiplexers. 


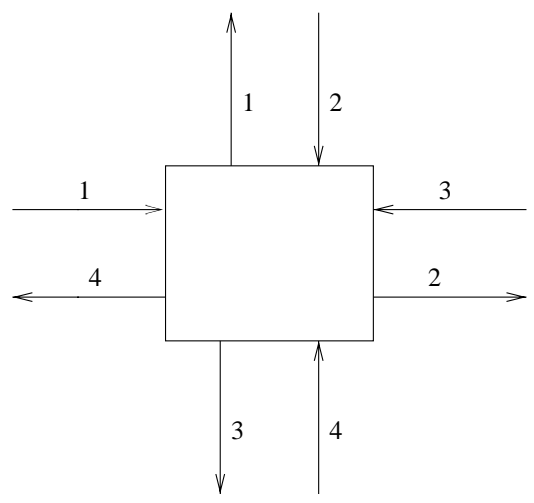

Figure 3.2: A circulator with 4 input and 4 output ports

\subsubsection{Multiplexers and Filters}

Multiplexing and filtering is an important part of optical transmission. Without these devices it would not be possible to do any switching nor to transmit several signals in the same fiber at the same time. Figure 3.3 shows three different applications for optical filters. The first one is a basic filter which reflects all the wavelengths but one. The second one is a more advanced device, a multiplexer. Multiplexer combines all the input signals from different fibers to one fiber. The third device is a wavelength router. With it several signals from several different fibers can be re-routed to several new fibers. The only limitation is that a single fiber cannot carry two signals with the same wavelength.

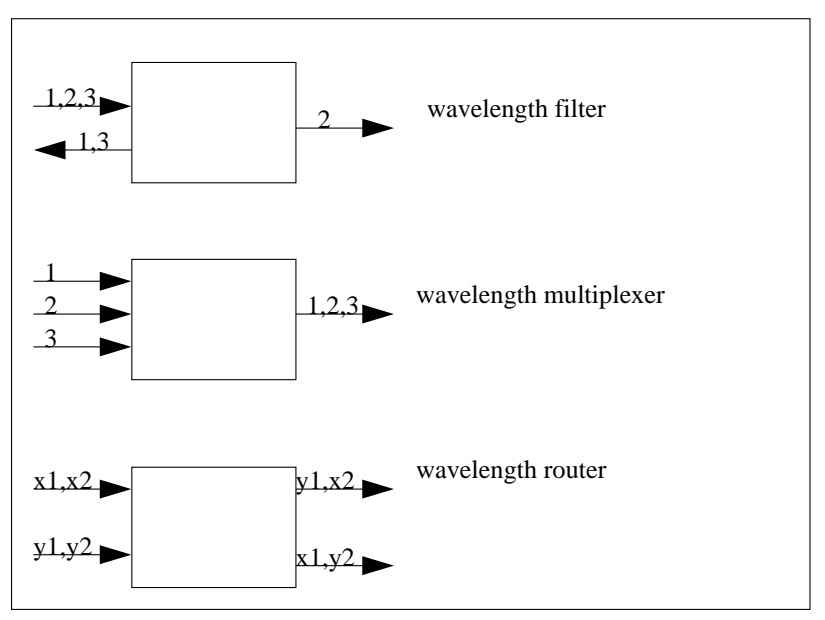

Figure 3.3: Different applications for optical filters in optical networks. Adopted from $[\mathrm{RS} 98]$

\section{Filtering}

To filter or multiplex channels which are based on wavelengths, one has to separate different wavelengths from the signal. There are many different ways to 
do this, but in principle they are all based on the same idea: some wavelengths are delayed in phase compared to other wavelengths. This is done by directing them through a longer path. In gratings the function is based on diffraction as shown in Figure 3.4. The signal is guided to a small hole, and it spreads to many directions. Because the path wavelength 2 travels is longer than the path of wavelength 1 , it is added in phase.

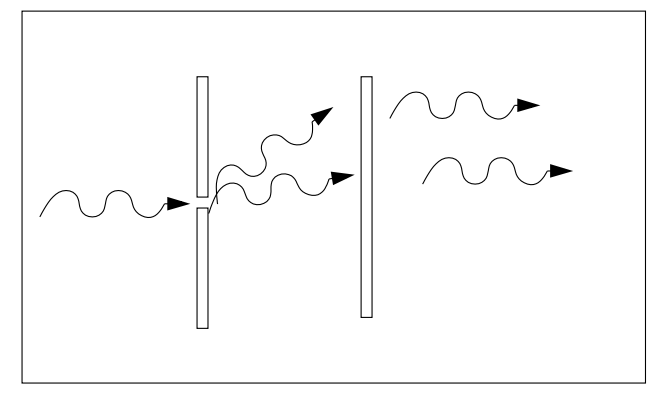

Figure 3.4: The operational principle of gratings

In arrayed waveguide grating (AWG) the idea is that signal with many wavelengths is copied to several fibers with different lengths and on each fiber all but one wavelength are rejected. The process is described in Figure 3.5. In principle the idea is same as in gratings: different wavelengths get different phases, when they are delayed differently.

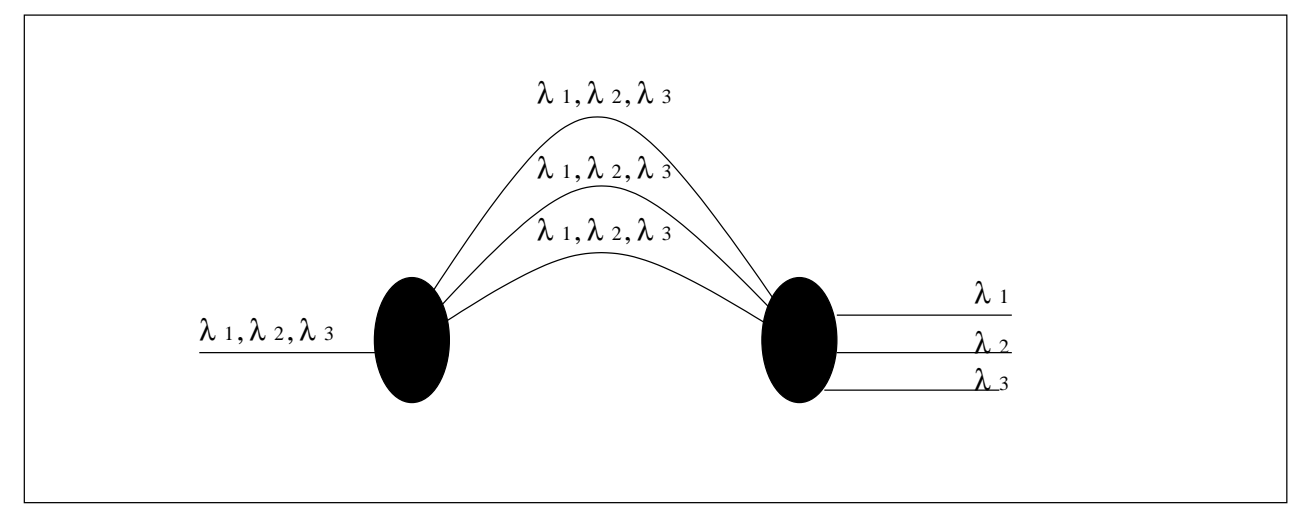

Figure 3.5: The operational principle of AWGs

Fabry-Perot filters are micro-optic cavities in which two mirrors are placed parallel to each other (see Figure 3.6). When a signal meets a mirror, a part of it continues through and the other part is reflected. The reflected signal travels straight to the other mirror and is guided back. It then travels through the same mirror wavelength 1 has already gone and will be added in phase.

The principle of thin films is basically the same as in Fabry-Perot filters. The difference is that in thin film devices the mirrors are realized by using mul- 


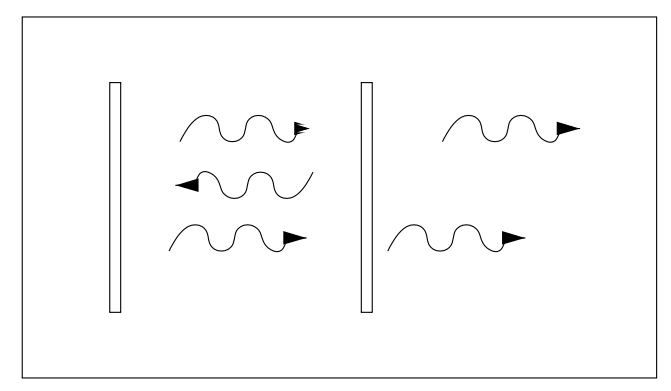

Figure 3.6: The operational principle of Fabry-Perot filters

tiple layers of reflective dielectric material.

The key parameters of filters are insertion loss and passband flatness. Insertion loss should be low and independent of polarization and temperature. Passband should be flat and passband skirts should be as sharp as possible. Figure 3.7 illustrates the signal. The flatter the passband is and sharper the passband skirts are, the smaller crosstalk energy passing through the adjacent channels is [RS98].

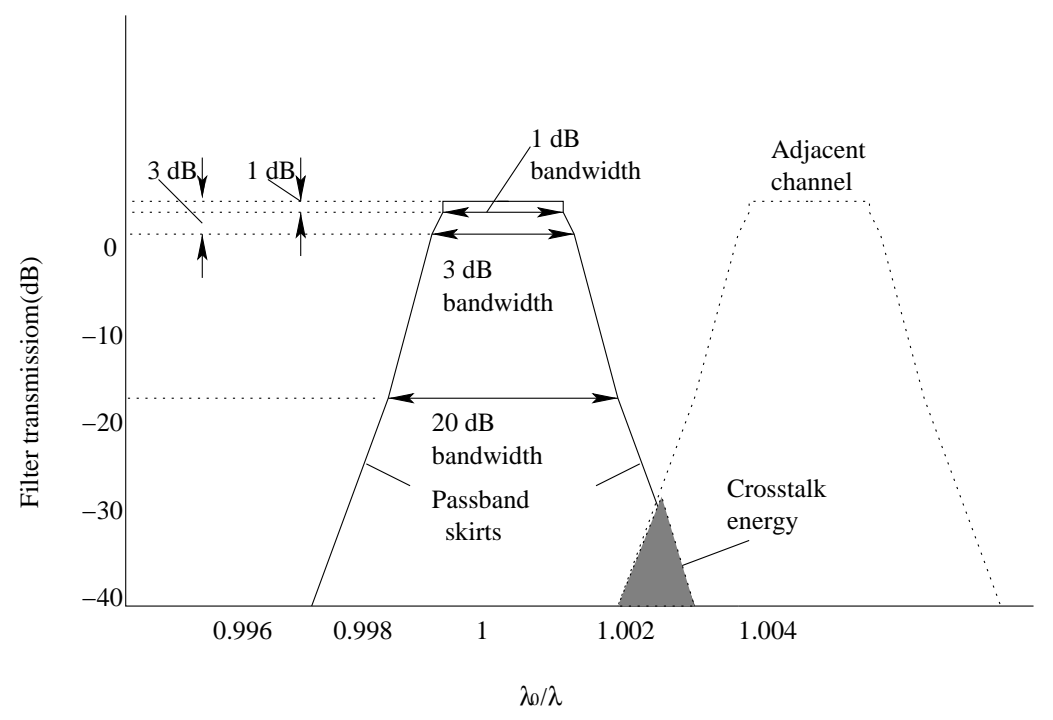

Figure 3.7: Characterization of some important spectral-shape parameters of optical filters. $\lambda_{0}$ is the center wavelength of the filter and $\lambda$ denotes the wavelength of the light signal, adopted from [RS98]

Fiber Bragg gratings are modified pieces of fiber that reflect one wavelength and pass through all the others. They are simple to manufacture and use and they have low insertion losses. AWGs are promising devices. They can be used in integrated optical circuits, while they can be easily combined with other functions. Additionally, they can be used as both a multiplexer and a demultiplexer. The disadvantage is their high temperature coefficient. The advantage of FabryPerot filters compared to many other filter types is their ability to be tuned to 
filter different wavelengths. Thin film devices are maybe the most promising filter components. Their passbands have flat top and sharp skirts. Their insertion loss is low and insensitive to polarization.

\section{Passive and Tunable Filters}

In principle filters reject all wavelengths except one. Passive filters are simple devices that have one fixed wavelength that they pass. Tunable filters can change the wavelength they accept. There are two parameters characterizing tunable optical filters. First the range of the wavelengths the filter can accept, and secondly the time required to tune from one wavelength to another.

\section{WDM Multiplexers and Demultiplexers}

Multiplexer is a device which combines several signals with different wavelengths to one fiber. Respectively a demultiplexer gets one signal as an input and by separating the different wavelengths from the fiber, assorts each wavelengths to its own output fiber. The purpose of these devices is to increase the capacity of a fiber by increasing the number of channels per fiber from one to hundreds.

\section{Add/Drop Multiplexers}

Add/drop multiplexers are devices that are used for adding or removing single wavelengths, i.e. channels, from a fiber without disturbing the transmission of other signals. Optical add/drop multiplexers are widely used in WDM networks.

\subsection{Active Optical Components}

\subsubsection{Amplifiers}

A signal attenuates while it travels through an optical fiber or through optical components. In order to travel over long distances the signal has to be amplified. Earlier the signals were converted to electrical form and regenerated. Today there are optical amplifiers and the signal can be transmitted over longer distances without conversion to electric form. Compared to regenerators, optical amplifiers are more flexible to changes in the bit rate. Additionally, they can be used to amplify several wavelengths at the same time.

\section{Fiber Amplifiers}

Erbium-doped fiber amplifiers (EDFAs) consist of a strand of fiber doped with Erbium atoms, pumping devices and simple optical components. They are alloptical devices. The operation of doped amplifiers is based on the stimulated 
emission (see Figure 3.8). The signal gets more energy if there are more transitions from a higher energy level to a lower than from the lower to the higher. The energy can be pumped to fiber by giving Erbium atoms more energy and lifting electrons to higher energy state. When electrons fall to lower level, energy is released and the signal is amplified.

1.)

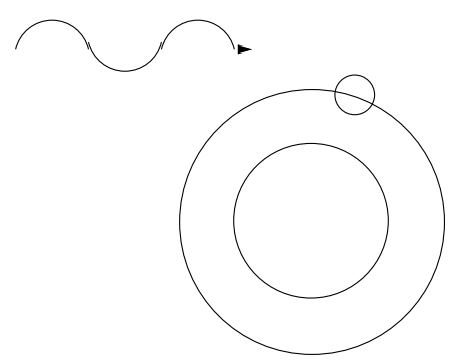

2.)

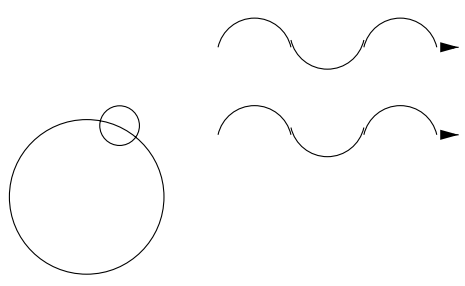

Figure 3.8: Stimulated Emission

For a long time the major problem with amplifying was the fact that different wavelengths were amplified with different gains. With EDFAs this is not a remarkable problem as the gain is relatively flat. Erbium-doped amplifiers were the first doped amplifiers constructed. Today there are also praseodymium-doped amplifiers, which can be used for similar purpose at $1300 \mathrm{~nm}$ wavelength band. The operational principle is the same as with Erbium doped amplifiers.

\section{Semiconductor Optical Amplifiers}

Semiconductor optical amplifiers (SOAs) are based on stimulated emission similarly as EDFAs. The difference is that instead of energy levels of dopant atoms, the process is based on electrons and electron holes in the semiconductor. The amplifier consists of two semiconductors separated with a band gap.

Actually, SOAs are not as good amplifiers as EDFAs. SOAs have wider bandwidth, i.e. they can be used to amplify more wavelengths. However, their output power is weaker, there is more polarization and coupling losses and they suffer from crosstalk. Still, there is a lot of interest for SOAs at the moment. SOAs are small compared to EDFAs which makes them suitable for building blocks for other devices, e.g. switches and wavelength converters.

\section{Raman Effect Amplifiers}

A Raman amplifier is not a compact device in the same way as the other amplifiers discussed here. The amplification process happens slowly in the transmission fiber over several kilometers. The main idea is that a light beam with lower wavelength and thus higher energy is guided to the same fiber with the signal to be 
amplified. The light beam with higher energy then delivers energy to the signal and signal is therefore amplified. Gain flatness is a critical issue with Raman Effect Amplifiers and several pumping bandwidths are needed to get the desired result. For further information, see [Int01].

\subsubsection{Transmitters and Receivers}

\section{Light-Emitting Diodes}

If the distance is short and the data transmission rate is low a cheap device called a light-emitting diode (LED) can be used as a light source instead of laser. LEDs have broad passband and low output power. A typical output power of LED is $-20 \mathrm{dBm}$, which is low compared to $0-10 \mathrm{dBm}$ output power of lasers [RS98].

\section{Lasers}

Figure 3.8 illustrates the stimulated emission. A photon interacts with the atom that is in the higher energy state. Then the electron drops down to the lower energy state and an equal amount of energy is released. Because of the stimulating photon the new photon produced has exactly the same direction, phase and wavelength as the first one. The stimulated emission is a necessity for the operation of a laser.

Additionally, a source of energy, a cavity filled with suitable material for the emission and two mirrors are needed (see Figure 3.9). The material can be solid, liquid or gas. It is suitable for a particular laser if the energy difference between the lower and the higher state of the atom is correct such that a photon with desired wavelength could be produced by stimulated emission.

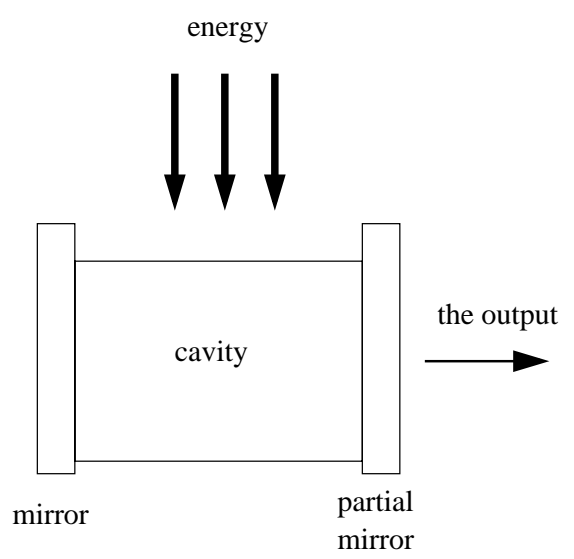

Figure 3.9: A laser

To produce a coherent light beam with the laser, energy is guided to cavity filled with the particular material. The electrons are then elevated to the higher energy state and photons are emitted spontaneously. Most photons emitted exit 
the cavity through the walls of the cavity. Some of them, however, confront the partial mirror (see Figure 3.9) with certain angle and are reflected back to the cavity. When this kind of reflected photon encounters an electron in the higher energy state stimulated emission happens and a new photon with exactly the same direction, phase and wavelength is produced. These photons continue toward the other mirror, stimulate new photons and are reflected back. When the photons meet the partial mirror, a part of them continue through and a part is reflected and stimulate new photons. Soon there are plenty of photons traveling in the cavity, and a great amount of light is also directed through the partial mirror.

In addition to the mentioned conditions, the length of the cavity has to be a multiple of half the wavelength of the output beam. Therefore the output light consist of only a limited number of wavelengths. However, the output beam of the lasers should be as narrow as possible and to improve the laser all these wavelengths but one should be rejected. This can be done by filtering or by using external cavity. Another alternative is to use a very short cavity. Today simple Fabry-Perot lasers, as the one discussed in the beginning of the section, are often replaced with distributed feedback (DFB) lasers. In these lasers the width of the cavity has periodic changes and the beam is reflected several times back and forth over short distances. This device can work as designed to be a narrow band single mode laser.

Another remarkable improvement to Fabry-Perot laser is the tunability. External cavity lasers can function as slow tunable lasers, if the length of the other cavity can be changed. Usually this is done by moving the mirror or grating which forms the external cavity. A faster way is to chance the bias current, which chances the refractive index. The disadvantage of this approach is variation in the output power. Another possibility is to use two independent currents, one for controlling the output power and the other for controlling the wavelength of the laser.

\section{Receivers}

The task of receivers is to convert optical signal to electronic form. A receiver consists of a detector, an amplifier and a decision circuit [RS98]. A photodetector generates an electrical current corresponding the optical signal received. This current is then amplified with amplifier and estimated with the decision circuit.

There are both fixed and tunable receivers. Fixed receivers select signals on a particular wavelength and block all the other signals on the fiber. Tunable receivers can change this wavelength.

\subsubsection{Wavelength Converters}

Wavelength converters are used for converting the wavelength of an incoming signal to a different wavelength. They can be used for instance as a part of a 
switch or cross-connect or in 3R regenerators. 3R regenerators are devices that regenerate signals amplitude and regenerate the signal also in time and frequency domains (see Chapter 5 for further information). Wavelength converters can be opto-electronic, or based on optical gating or wave mixing. Opto-electronic converters are devices which convert signal to electronic form, regenerate and retransmit it. The problem with opto-electronic converters is that they require fixed data rate and format. The two other possibilities are discussed here in more detail.

\section{Optical Gating}

There are two possibilities to use optical gating: cross-gain modulation and crossphase modulation. The first is based on the fact that the gain of a SOA dependents on input power. A low power probe signal with desired wavelength is sent into the SOA. Because the probe signal has a low gain compared to the input signal, it will experience high gain when the gain of the input signal is high (state 1) and low gain when gain to the input signal is zero (state 0 ).

In cross-phase modulation the phase of the probe signal is changed on basis of the input signal. The phase modulation is then converted into intensity modulations by using interferometer. Cross phase modulation is the more attractive alternative of these two. It requires less power and it has a better extinction ratio. Additionally, pulse distortion, from which cross-gain modulation suffers, is not a problem but this feature is the operational principle of the device.

\section{Wave Mixing}

In wave mixing the idea is to construct a desired signal by using probe signals with such wavelengths that together with the input signal they form another signal with desired wavelength. The advantage of this approach is the transparency, and the disadvantage is the fact that there is additional signals in the output and these signals have to be filtered out. 


\section{Chapter 4}

\section{Switching}

\subsection{Introduction to Switching}

A switch is a word with two partly different meanings. A brief definition is that a switch is a device used for switching signals from input ports to output ports. However, a switch can mean a simple device with few components or it can be a complex device that, e.g., consists of complex controlling block, delay line buffers, filters, wavelength converters and simple switches. Even a simple staggering switch illustrated in Figure 4.4 consists of a delay line buffer and two space switches. Furthermore, a switch can mean both a component and an entire node in a network. This chapter concerns mostly network node type switches. To understand their function, two basic switching components, i.e., space switch and wavelength router, are first introduced below.

\subsubsection{Space Switch and Wavelength Router}

Space switches and wavelength routers are basic components of an optical switch. A space switch simply switches signals from each input to one of the outputs. There are several ways to implement a space switch, but a semiconductor optical amplifier (SOA) technology seems to be the best alternative. A space switch based on SOA technology is illustrated in Figure 4.1. SOAs and SOA gates are basic components of optical networks. In addition to switches they can be used, e.g., in wavelength converters.

An $N \times N$ SOA gate based switch, illustrated in Figure 4.1, consists of $N$ $1 \times N$ splitters, $N^{2}$ SOA gates and $N N \times 1$ combiners [Muk97]. Each input $i$ is split to $N$ gates. If the signal is to be switched to output $j$, the gate $j$ is in on state and the other gates are in off state. All gates with the same index are connected to a combiner, which leads to the output port corresponding to that index. If only one packet at time can be processed at that output port, then only one gate at time can pass a packet to each output port.

A wavelength router can be non-reconfigurable or reconfigurable [Muk97]. The wavelength router illustrated in Figure 4.2 is non-reconfigurable. A signal from input $i$ with wavelength $j$ is always directed to the same output $k$. An exam- 


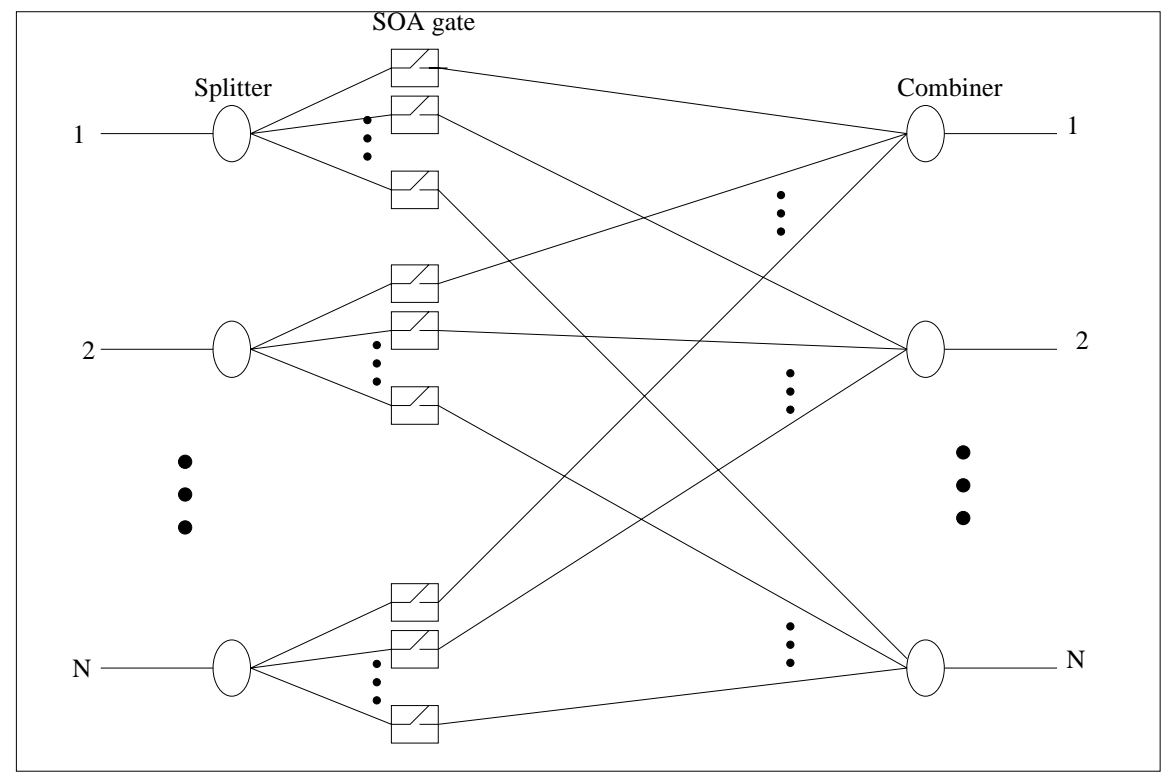

Figure 4.1: A SOA gate based switch

ple of this kind of router is an arrayed waveguide grating multiplexer (AWGM) [Muk97]. An AWGM consists of two star couplers and an arrayed waveguide grating between them. Often, (e.g., in $\left.\left[\mathrm{HNC}^{+} 99, \mathrm{CHA}^{+} 01\right]\right)$, AWGM is called an AWG wavelength router or simply AWG. The star coupler splits signals from input ports to all the waveguide gratings, which have different lengths. The delay signal experiences depends on the length of the waveguide and the wavelength. At the second star coupler, the phase differences cause that the signal will constructively recombine only at a single output port [Muk97].

Although a non-reconfigurable wavelength router does not have a switching property, it is widely used in wavelength routing optical packet switches. The basic idea is that every packet is first converted to a certain wavelength and then directed to AWGM. Because AWGM chooses the output port of each packet according to the input port and the wavelength, each packet will be switched to predeterminated output port.

A reconfigurable wavelength router is capable of switching packets by itself. A non-reconfigurable wavelength router can be modified to be reconfigurable by adding space switches between demultiplexers and multiplexers of nonconfigurable router. The operational principle is the following. First signal from each input port is demultiplexed and each signal with wavelength $i$ is guided to switch $i$. Switches are capable of switching a signal from any input to any output. Finally a multiplexer at output port $j$ gets $M$ signals with $M$ wavelengths, multiplexes them and sends that combined signal to output fiber $j$. 


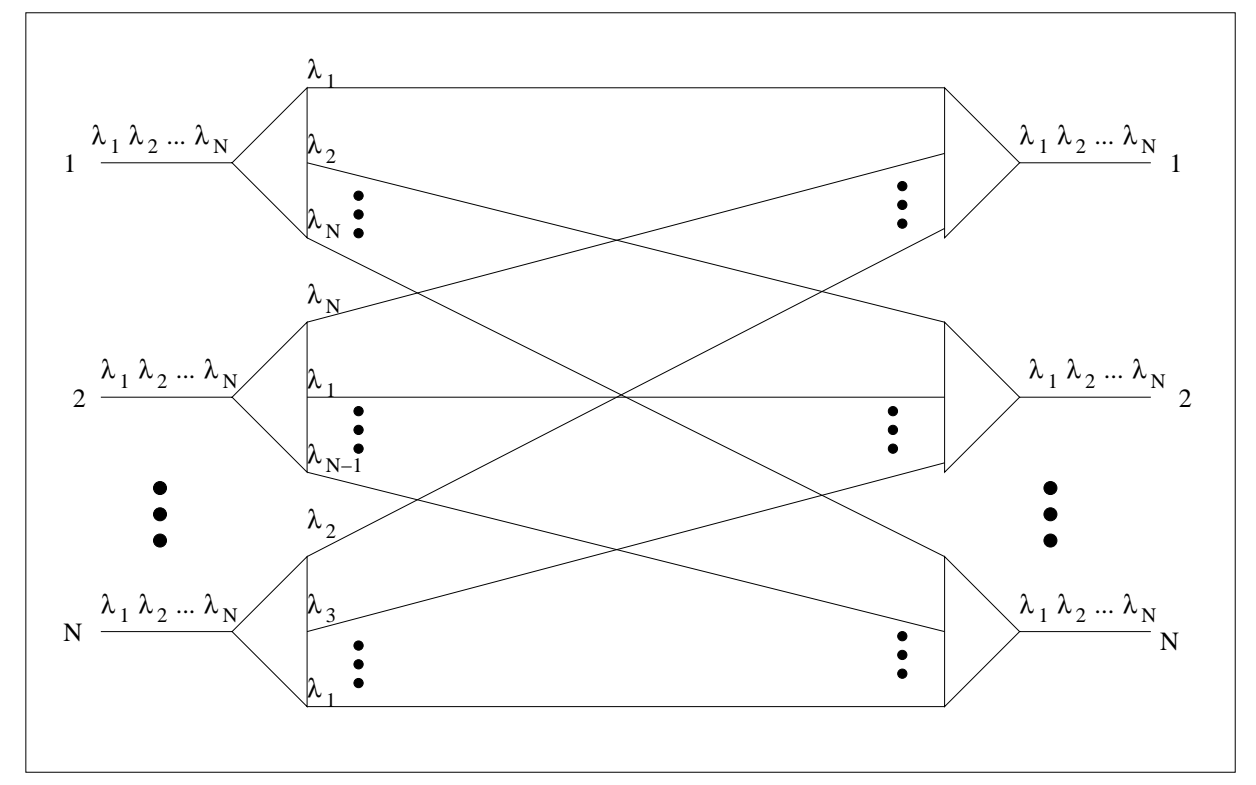

Figure 4.2: A wavelength router, adopted from [Muk97]

\subsubsection{Classification of Switches}

Switches can be classified to optical and electronic ones. Electronic switches are more mature devices compared to optical switches and implementing them is easier. In optical networks they have two serious problems: electrical switching is slow compared to propagation speed in optical fiber and opto-electronic and electro-optical conversion needed in switches delays the signal even more. Optical switches are devices in which the data is maintained in optical form. Controlling is naturally performed electronically.

Switches can also be divided into relational and logic devices [Muk97]. First class includes switching elements, in which the content of channels does not affect to the switching, e.g., couplers. The other type of switching elements, i.e. logic devices, are considered in this chapter. In logic switches the content of channels (e.g., headers of switched packets) controls the switching between inputs and outputs. A more familiar classification of switches is classification to circuit and packet switches. Relational devices are used for circuit switching and logic to packet switching. Circuit switches are easier to implement because the switching speed needed is slower than in packet switches, but on the other hand they are inefficient when compared to packet switches.

The third alternative is classification on the basis of the fidelity of switching. The coarsest class are space switches that switch the content of an entire fiber from an input to one of the outputs. These switches are unable to switch a wavelength from an input to output $i$ and another wavelength from same input to output $j$. Some times these coarse switches are called optical crossconnects [Muk97] but usually optical crossconnects mean circuit switches capable of switching different wavelengths to different destinations $\left[\mathrm{VKM}^{+} 01\right]$. In WDM networks 
more accurate switches are needed (e.g., OXC capable of separating wavelengths or in packet network optical packet switch). However, optical space switches are important components of more complex switches and systems.

In this chapter optical packet switches are studied. Most architectures covered here are 1-wavelength architectures. Each input port can take only one packet at a time, which means that each fiber can carry only one wavelength at a time. This kind of switches cannot be used in practice, because WDM will be used in packet networks. However, these switches give a good idea of general structure of a packet switch. A few switches suitable for WDM networks are also reviewed.

\subsection{Packet Switch Architectures}

Generally an all-optical switch consists of four parts: an input interface, a switching fabric, an output interface and a control unit [YMD00a, OSHT01]. In Figure 4.3 control unit is drawn inside other blocks. In the figure the control unit consists of electrical components.

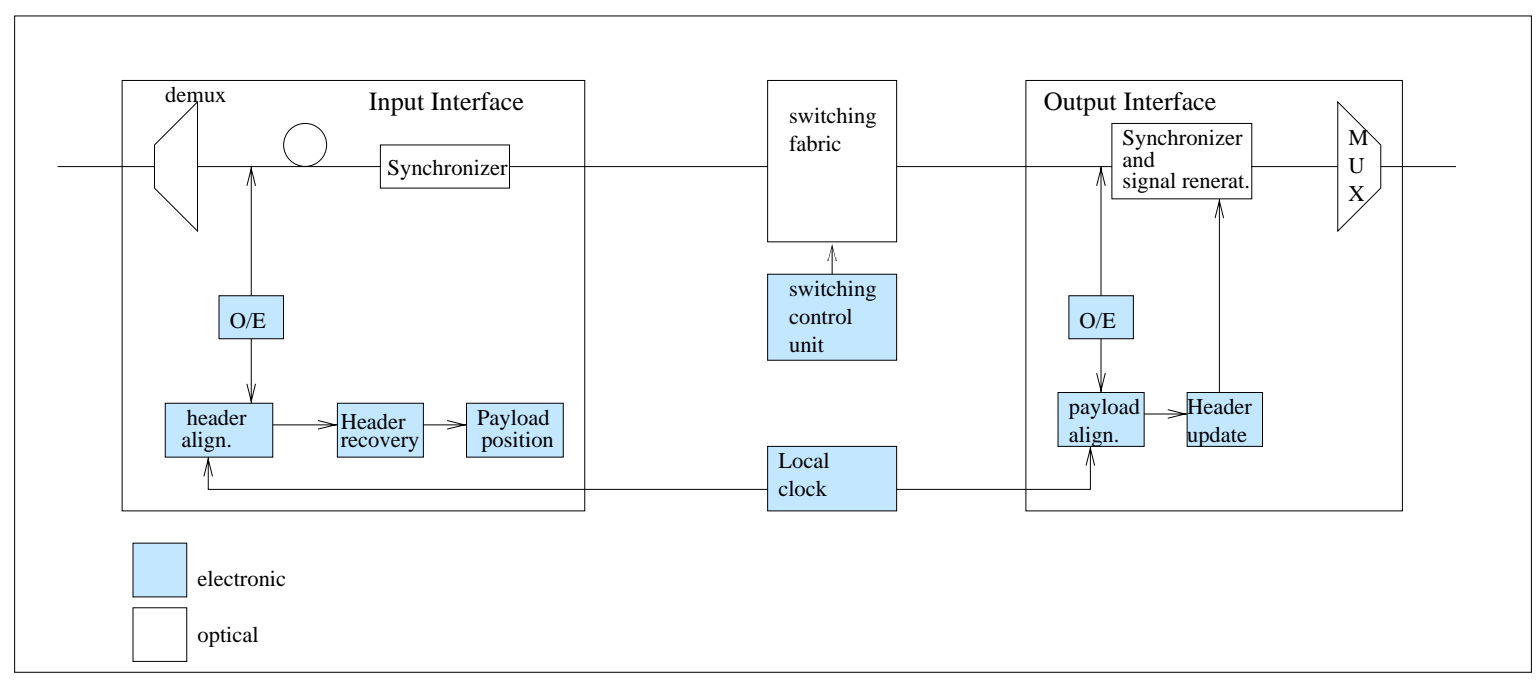

Figure 4.3: The main parts of a switch, adopted from [LPTS01]

An input interface takes care of synchronization and delineation of packets. If necessary it can also amplify packets before switching. The packet delineation is necessary for header recognition, payload detection and header rewriting. Additionally delineation is needed for synchronization of the packets. The input synchronization is required to compensate the slow jitter. The synchronization is done by delaying packet for a certain time.

Switching fabric routes packets to desired output ports on the basis of the information contained by the packet header. It also performs contention resolution. 
Contention resolution can be done with optical fiber delay lines, by using wavelength converters or by using both. In principle buffers or wavelength converters that are used for contention resolution can be placed either ahead the switching components or after them. Switches using the former alternative are called inputbuffered and switches using the latter are called output-buffered. The contention resolution schemes are discussed in more detail in the next section.

An output interface regenerates or amplifies data, rewrites headers and performs output synchronization. An output synchronizer is required to compensate for delay variation inside the switching node. A control unit reads the packet headers and based on that information controls the switching fabric. The packet header can be managed either optically or electronically. The latest switch architectures proposed are based on the idea that payload remains in optical domain but header is processed electronically. An exception is architecture called data vortex [YBHJ01]. In data vortex networks routing is done with WDM encoded header bits processed with wavelength filters. The control unit including header processing functions is usually electronic while the other units are optical. Traditionally, optical packet switches can be classified, based on the structure of the switching fabric used, to the following three classes: space switching based switches, broadcast-and-select switches and wavelength routing switches [TZ99]. Recently photonic slot routing and similar slot based network architectures have gathered interest [SdGD01, ZJM00, DvBK $\left.{ }^{+} 01\right]$. Switches of the three base types and also switches used in these slotted networks are discussed next.

\subsubsection{Space-Switching-Based Switches}

Space switches are important components of wavelength routing or broadcastand-select type switches. These switch architectures are described later. Optical packet switches mainly based on space switching are studied in this subsection. The main problem of space switching based architectures is the increasing need for SOA gates when the number of inputs and outputs grows.

\section{Staggering Switch}

The staggering switch illustrated in Figure 4.4 is probably the best known space switching based switch. It is a simple switch consisting of two space switches and delay lines between them. The first space switch directs packets to right delay lines, such that packets destined to same output will be delayed different times. The second switch then switches packets to right output ports. The staggering switch can be improved by replacing the delay lines with recirculating delay lines [TZ99]. That is, packets can be switched back to delay line buffer several times. This way the maximum time a packet is buffered can be increased without a need for longer delay lines. On the other hand recirculateted packets has to be amplified, which leads to increase of noise [YMD00a]. If recirculated buffers are used, only one space switch is needed. The staggering switch does not maintain the FIFO queuing discipline. In staggering switch inputs and outputs can contain 
one packet at time.

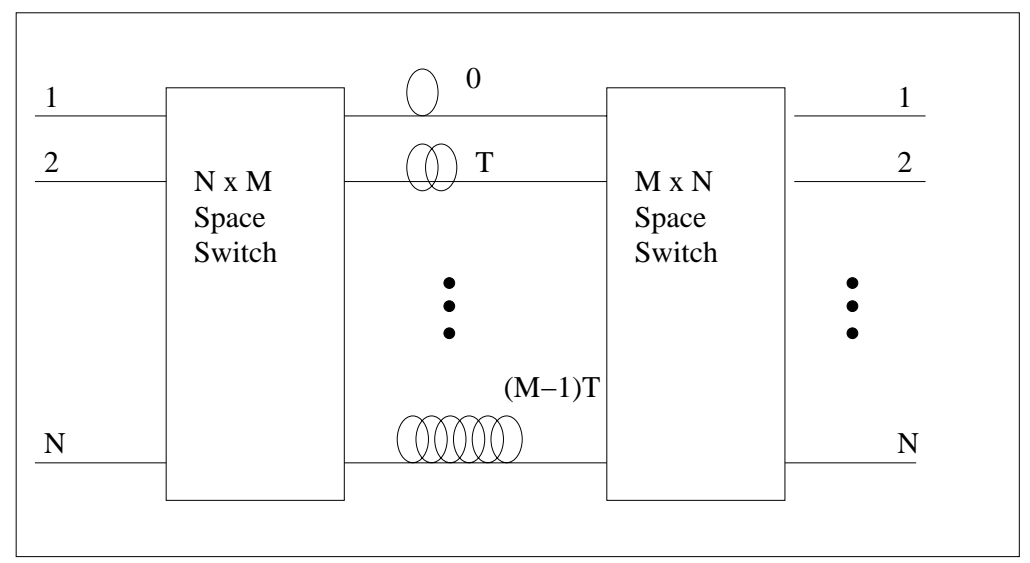

Figure 4.4: The structure of staggering switch, adopted from [TZ99]

\section{Output-Buffered Photonic Packet Switch}

Another space switching based architecture is an output-buffered photonic packet switch using wavelength-routed packet buffer [TZ99]. The switch is illustrated in Figure 4.5. Each input is converted to an individual wavelength. Therefore several packets traveling to same output can be multiplexed together in the space switch. Packets traveling to the same output are given different delays in the buffering part of the switch. The first AWGM delivers packets to right delay lines and the second AWGM directs packets to right outputs, i.e., to same outputs as the input ports where packets arrived to this block. Inputs and outputs can contain one packet at time.

\section{Bufferless Space-Switching-Based Switch}

In [EL00] a bufferless space-switching-based architecture was studied. The switch is illustrated in Figure 4.6. Instead of buffers, contention resolution is done with tunable wavelength converters. Number of wavelength converters can be smaller than number of wavelengths, because wavelength converters are shared. First, arriving packets are demultiplexed and split to number of fibers. Each fiber has an optical gate. One of the gates is in on state and the packet is directed to an output port either directly or through a tunable wavelength converter. Packets arriving at an output port are coupled and sent to an output fiber. In this model each input and output can contain $N$ packets at the same time.

Similar models, where contention resolution is performed with buffers or with deflection routing have been proposed [XPR01]. However, contention resolution with wavelength conversion seems to be more efficient. 


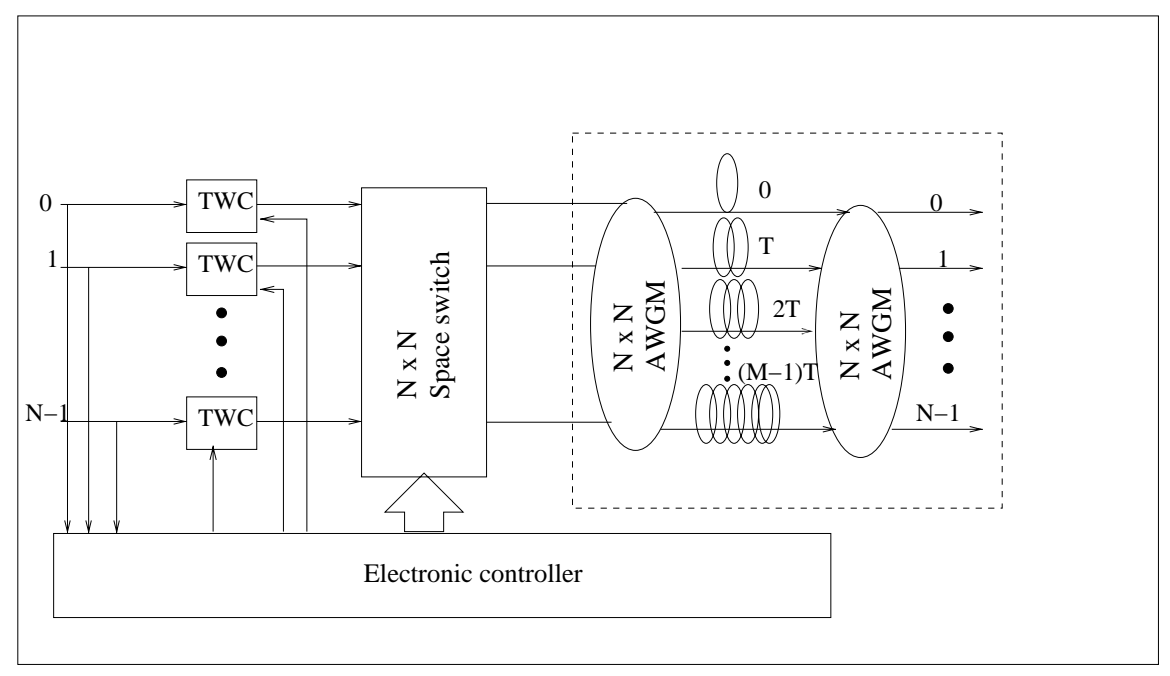

Figure 4.5: The structure of output-buffered photonic packet switch using wavelength-routed packet buffer, adopted from [TZ99]

\section{The DAVID Switch}

The architecture of the switch is illustrated in Figure 4.7. Each input fiber has several wavelengths. At the input ports packets at these channels are demultiplexed and they are guided to switching matrix that is illustrated in more detail in Figure 4.8. In the switching matrix wavelength conversion is performed in order to handle a situation where there are several packets at the same output port or in the same delay line at the same time. Each packet travels through the switch with the same wavelength. At the output port the packets wavelength is converted to desired output wavelength. The wavelength packet has inside the switch is independent of the external wavelength packet has outside the switch. In this switch packets can be of variable lengths.

In the switching matrix packets are first converted to a wavelength that guarantees that there will be no contention. The number of wavelengths used inside the switch, $W$, can be less than or equal to the number of external wavelengths, $M$. The delay line buffer is implemented with cascade of $k$ stages of $m$ delay lines. At each state packets from the delay lines are directed to a space switch through a wavelength splitter. The splitter consists of a combiner and wavelength demultiplexer. This architecture guarantees that any output of stage $i$ can be switched to any input of the stage $i+1$. Each stage has different delays such that, delay line $i$ at state $k$ produces delay $i m^{k-1}$. The output state chooses right packets to the appropriate outputs according to the wavelength and the delay line. When the right output is reached, packets are converted to the right wavelength and directed to the output fiber. The architecture is FIFO scheduled, i.e., packets arrived at output ports in FIFO order. 


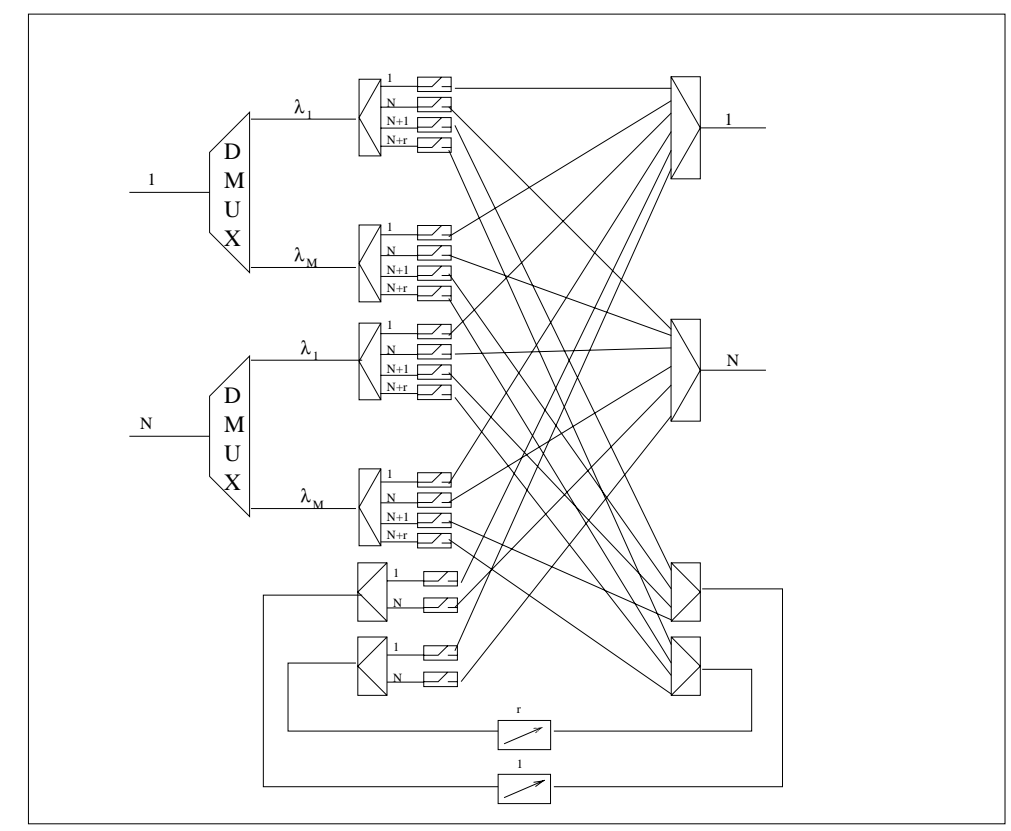

Figure 4.6: Bufferless space switch with tunable wavelength converters, adopted from $[$ EL00]

\subsubsection{Wavelength Routing Switches}

Wavelength routing switches are switches, in which the desired output ports are marked by wavelength conversion at the input ports. Each packet is given a wavelength corresponding to the output port and the switching is done according to the wavelength.

\section{Output-Buffered Wavelength Routing Switches}

A wavelength routing switch was proposed as early as 1992 by Gabriagues and Jacob [TZ99]. The structure of the switch is presented in Figure 4.9. The switch has three optical functional blocks: a packet encoding block, a buffering block and a packet demultiplexing block. The switch is controlled with electrical controller. The first block consists of $N$ tunable wavelength converters (TWCs), which convert packets to wavelengths corresponding to desired outputs. Buffering stage consists of an $N \times K$ SOA gate switching matrix and $K$ optical delay lines. Switching matrix directs packets to desired delay lines. Electronic controller takes care that packets destined to each output port follow FIFO scheduling principle. The third block includes a $K \times N$ star coupler and a set of $N$ passband filters. Star coupler multiplexes all packets from different delay lines to all output ports. Filters choose packets that have wavelength corresponding to that particular output. Number of SOA gates needed increases in proportion to product of $N$ and $K$. Each input and output can contain one packet at time.

Another form of wavelength routing switch is the frontiernet photonic packet switch implemented in early 1990's [TZ99]. The heart of the switch is an AWGM 


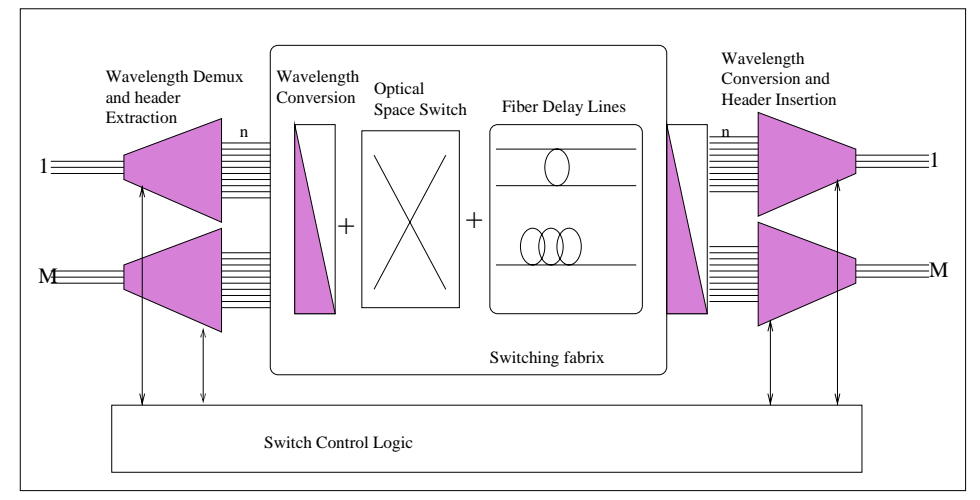

Figure 4.7: The architecture of the optical packet switch implemented in the DAVID project [CCR02]

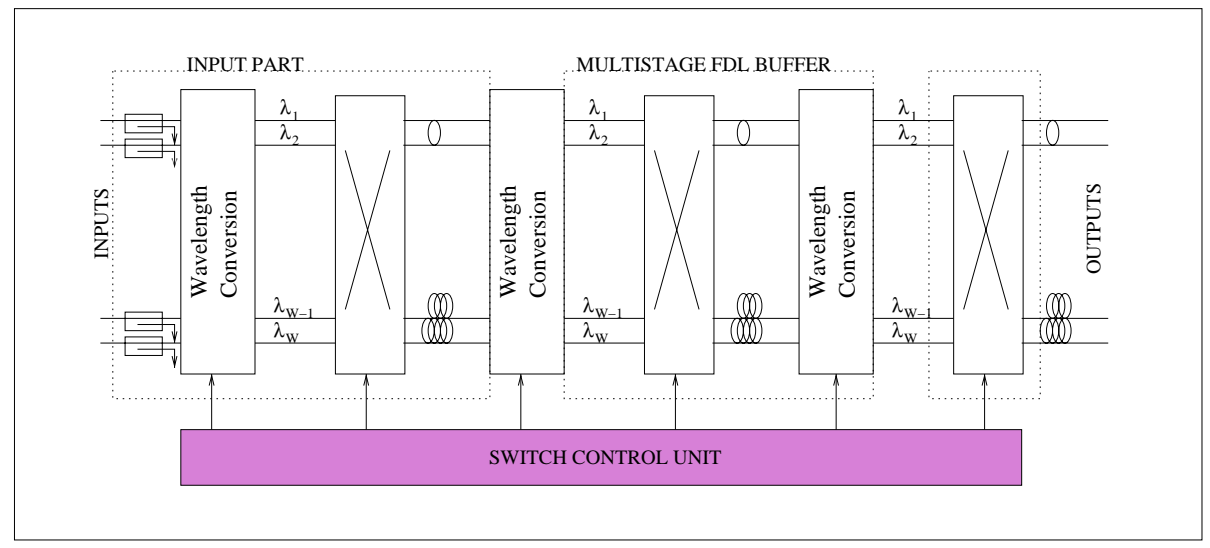

Figure 4.8: The architecture of the switching fabric of the switch implemented in DAVID project [CCR02]

(arrayed waveguide grating multiplexer), i.e., a non-reconfigurable wavelength router. $N \times N$ switch uses $N$ wavelengths to route the packets. In AWGMs a packet is routed according to the input port and the wavelength. Therefore each input has to have one individual wavelength for each output. Additionally, packets directed from different inputs to the same output have different wavelengths, e.g., a packet from input 1 to output 1 gets wavelength $\lambda_{1}$, a packet from input 2 to output 1 gets wavelength $\lambda_{2}$ and a packet from input 1 to output 2 gets wavelength $\lambda_{2}$. Each input and output can contain one packet at time.

First packets are guided through tunable wavelength converters (TWCs), then through AWGM to one of the cascaded recirculating buffers. TWCs choose desired wavelengths for every packet and the AWGM then routes packets to desired output according to the wavelength. Because the AWGM is capable of routing several packets to the same output at the same time, the possible contention occurs at the output port. Cascaded recirculating buffers take care of that kind of situations. If there is contention, other packets but one are buffered. 


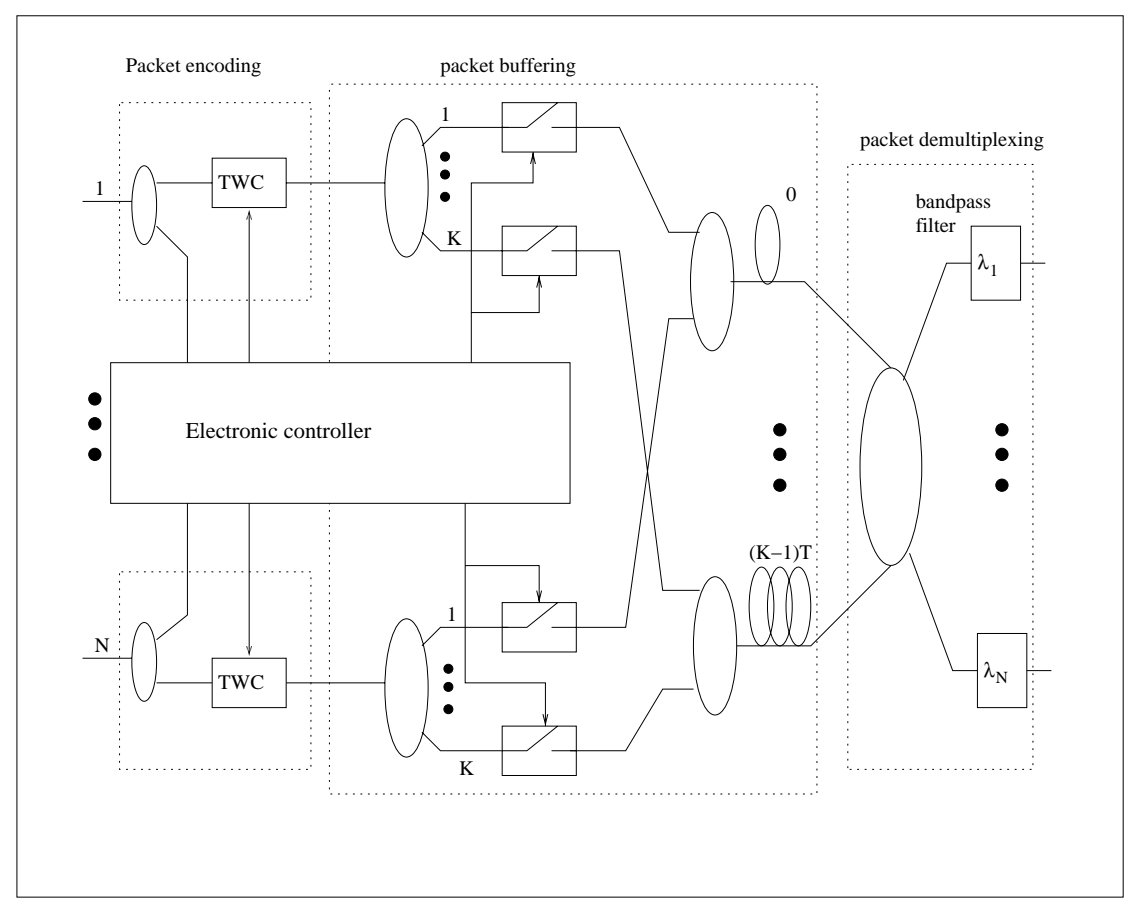

Figure 4.9: The main parts of a wavelength routing switch, adopted from [TZ99]

The idea of a cascaded recirculating buffer used in frontiernet switch is the following. There are $M$ recirculating buffers. Each buffer consists of an optical fiber loop, two SOA gate switches, and a $3 \mathrm{~dB}$ coupler. As explained in Chapter 3 the $3 \mathrm{~dB}$ coupler delivers two input signals equally to two output ports so that both outputs get a combination of the input signals. The first SOA gate determines, if a packet stream is directed to the loop, and the second determines, if the packet stream in the loop is directed to the output or if it is to be delayed once again. An arriving packet stream consisting of packets arrived at the same time slot is directed to a free loop as close to the output port as possible. If buffers are all empty the signal is also copied to the wavelength selector at the output port. At every time slot wavelength selector selects one packet to the output and the other packets are dropped out from the selector. In the next time slot the packet stream in the loop nearest to the selector is copied to wavelength selector again. When all packets in the loop nearest to the selector are switched out, the packet stream from each loop is copied to the next loop, i.e., at every time slot packet streams from each loop $i$ are moved to loop $i-1$ if (and only if) all the packets in the loop 1 are forwarded. The electronic controller calculates when packets from loop 1 are all forwarded and the signals from other loops can be forwarded to next recirculating loops. 


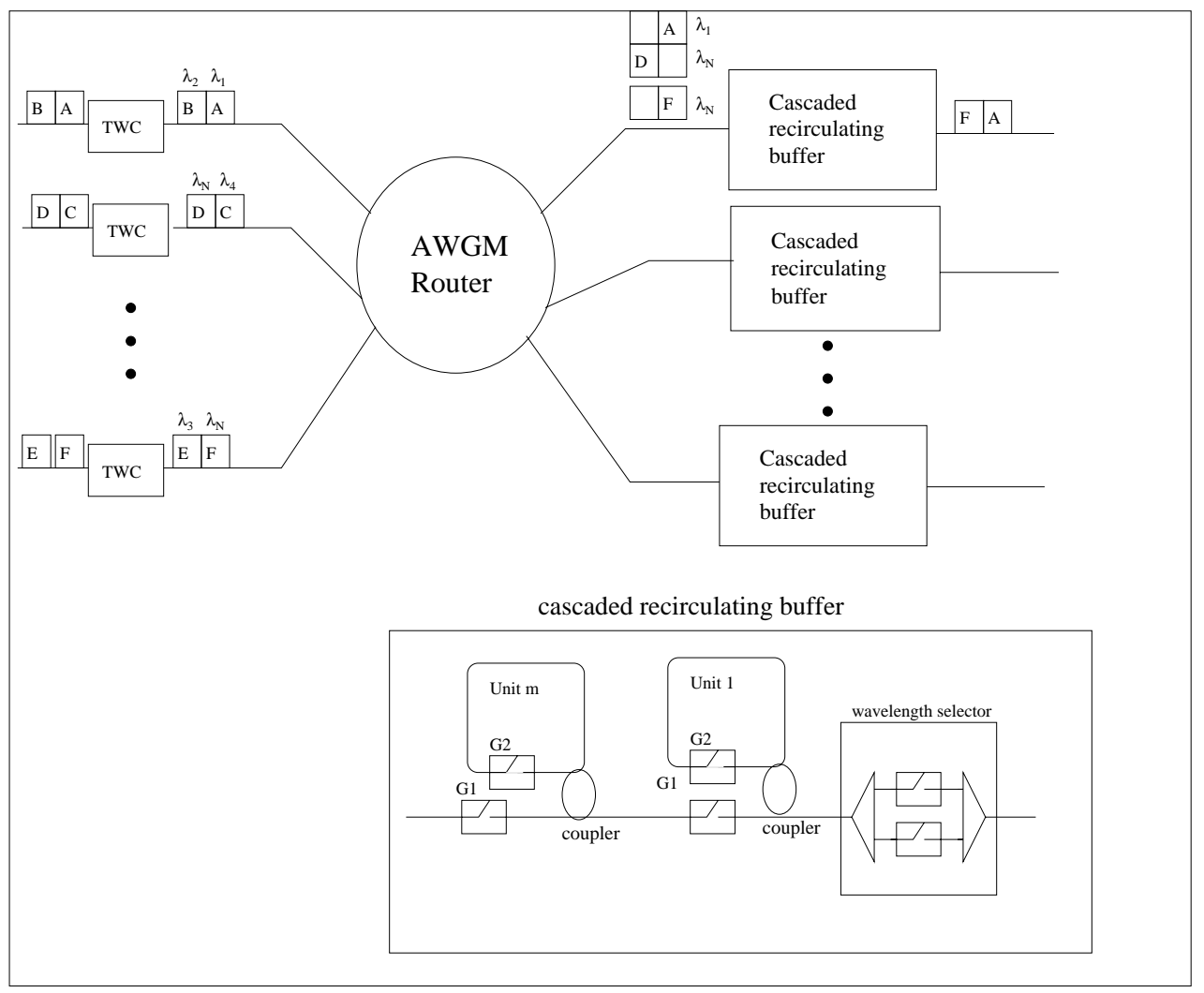

Figure 4.10: The frontiernet photonic packet switch

\section{Input-Buffered Wavelength Routing Switches}

The three switch architectures above were output-buffered i.e., switching was performed before buffering. The other type of wavelength routing switches are input-buffered switches. Wavelength routing switch implemented during KEOPS is illustrated in Figure 4.12, and is also described in Chapter 5. The switch slightly differs from the output-buffered switch presented above. The main difference between these two switches is that in the switch above the switching control is done before buffering and in the KEOPS switch the switching control is performed after the buffering. While in the switch above there is only one delay line set with $K$ delay lines of different lengths, the KEOPS wavelength routing switch has $N$ sets of $K$ delay lines. In the switch above the switching is controlled with gates that choose to which delay line a packet is directed.

In KEOPS switch each packet is delivered to all kind of delay lines, i.e., each packet has its own $K$ delay lines. Then output of each delay line set is multiplexed, one packet is chosen and with multiplexer demultiplexer set, corresponding to star coupler of the switch above, packets are copied to each output. One packet is chosen according to wavelength and the others are filtered out. In KEOPS wavelength routing switch each input and output can contain one packet at time.

Another switch model is input-buffered presented in [TZ99]. As illustrated 


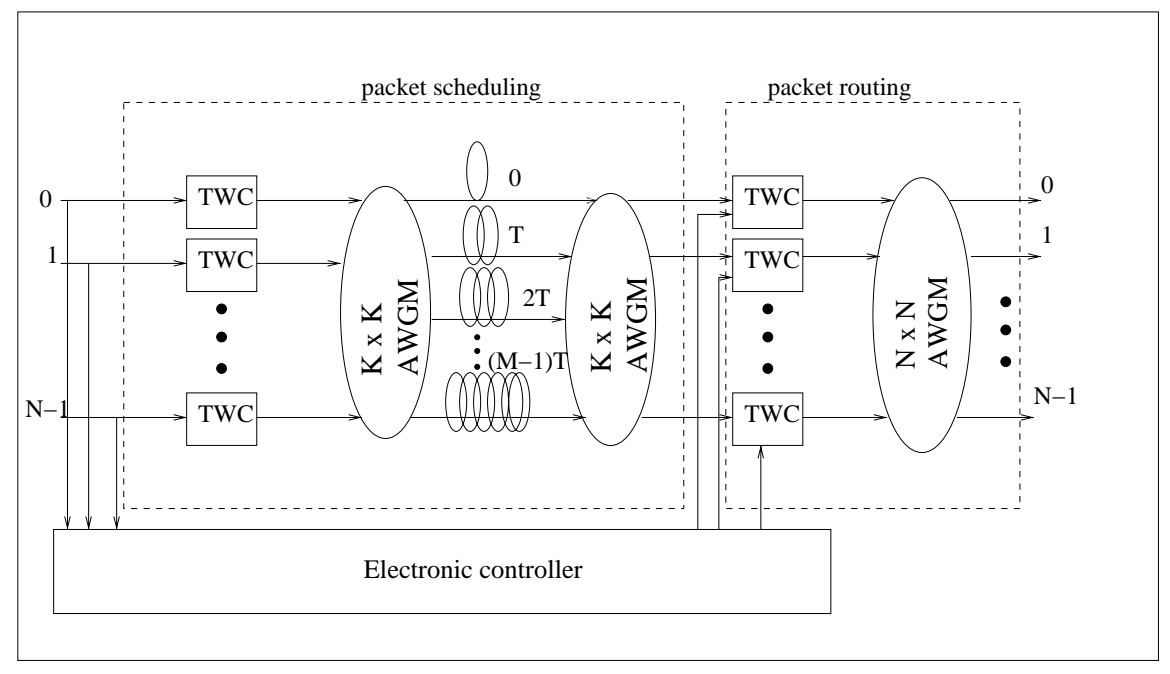

Figure 4.11: The input-buffered wavelength routing switch, adopted from [TZ99]

in Figure 4.11 packets are first buffered in a packet scheduler. Each packet gets desired wavelength and is then buffered in the wavelength routing buffer. The buffer consists of a pair of AWGMs connected with fiber delay lines. The first AWGM routes a packet to desired delay line according to the wavelength and the input port of the packet. Only one packet can be in an input or output of the next block at the same time. However, several packets can be put to a single delay line at the same time if they have different wavelength. The second AWGM directs packets to the output with same index than the index of the input port. Packet routing block then takes care of packet routing such that a packet is first given the right wavelength and then it is directed with an AWGM to output port corresponding to the wavelength. It is also possible to increase the switching capacity by modifying the switch by replacing the last AWGM with two smaller ones and adding $1 \times 2$ space switches before them [TZ99]. Each input and output can contain one packet at time.

In the WASPNET project three input-buffered wavelength routing switches were implemented. The first switch, called feed-and-forward AWG-based optical packet switch, is very similar to the input-buffered switch described above. The main difference is that in the switch described above there is one delay line set that can buffer several packets with different wavelengths at the same time while in the switch implemented in WASPNET project there is an own buffer for every output of the first AWGM. In input-buffered node switching is performed separately after the buffering while in a feed-and-forward AWG-based optical packet switch switching is performed simultaneously with buffering. In the feedand-forward switch only two AWGMs were needed, but on the other hand the number of TWCs and fiber lines is manyfold compared to input-buffered switch.

The feed-and-forward switch was further developed by first replacing the delay line sets with recirculating ones. Finally a switch known as WASPNET switch was implemented. A one wavelength model of the switch is illustrated in Fig- 


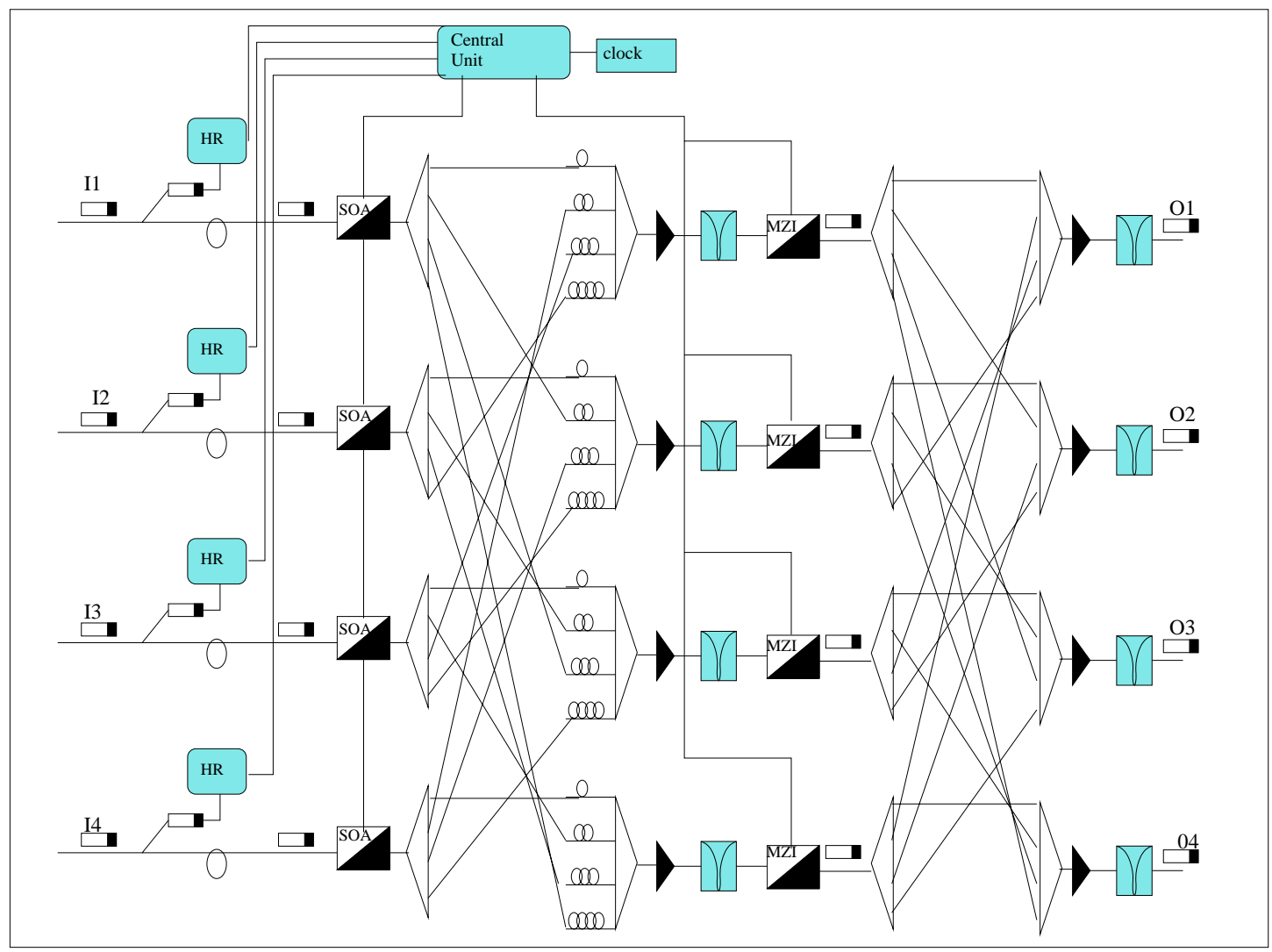

Figure 4.12: Architecture of a wavelength routing switch, adopted from [CJR ${ }^{+} 98$ ]

ure 4.13. First packets are converted to a wavelength according to which output port of the first AWGM is chosen. If the packet need not be buffered, it is guided through a TWC to a second AWGM that switches it to the right output port according to the wavelength. If buffering is needed the packet is switch to one of the ports that leads to delay lines. There it is again converted to a certain wavelength and split to all the delay lines. At the other end of the delay lines one of the packets with different wavelengths is chosen, converted to a some wavelength and guided back to the first AWG. This figure illustrates the model where each input port has only one wavelength, i.e., each input and output can contain only one packet at time. The actual WASPNET switch consists of multiple demultiplexers, multiplexers and planes each operating at a single wavelength. The figure above demonstrates one plane of the switch. In WASPNET switch each input is demultiplexed and each demultiplexer sends packets with wavelength $i$ to plane $i$. Similarly, each output is obtained by multiplexing a combination of the outputs of the planes. 


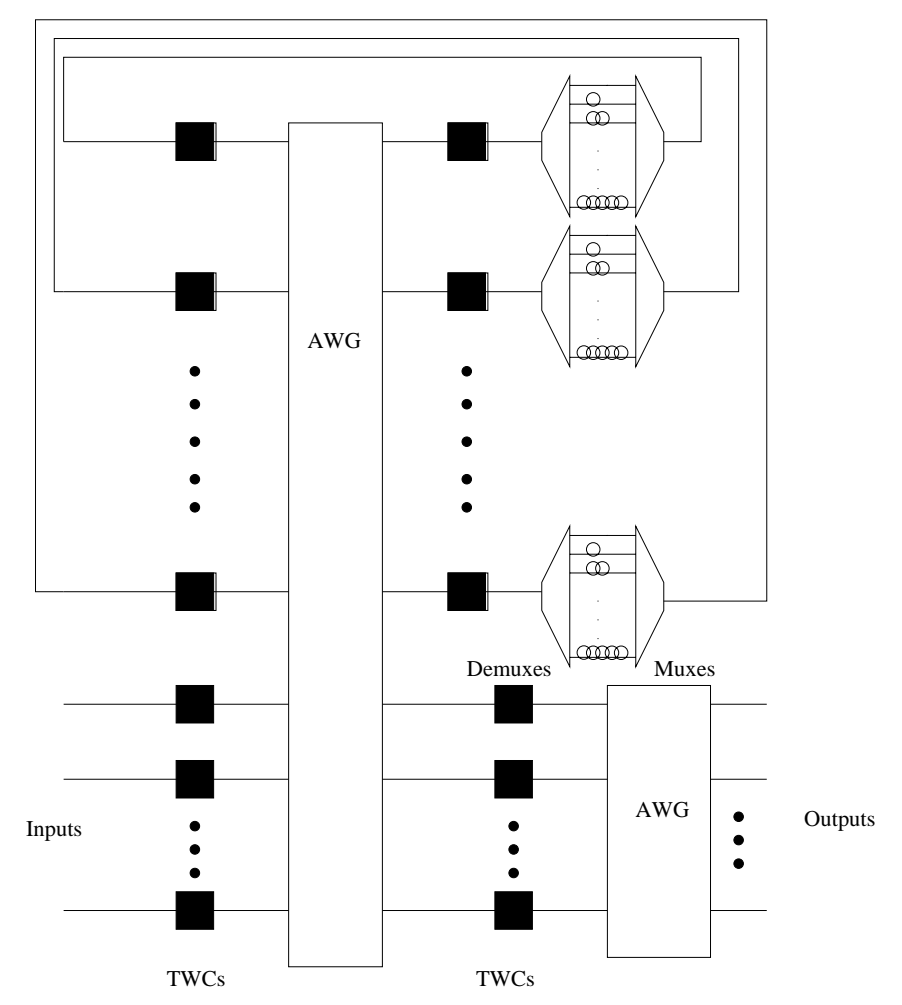

Figure 4.13: WASPNET switch, adopted from $\left[\mathrm{HNC}^{+} 99\right]$

\subsubsection{Broadcast-and-Select Switches}

A broadcast-and-select switch is a common switching architecture. This kind of switches have been implemented in many projects. Broadcast-and-select switches do not need tunable components as much as wavelength routing switches [TZ99]. Another benefit of broadcast-and-select switches is their ability for broadcast and multicast. The operational principle of broadcast-and-select switches is that the information from each input is combined to one signal that is copied to each output. Each output block then filters the desired information (packet) from the signal and drops the other packets. The switch can be wavelength based (WDM) or time based (TDM) or it can use both alternatives [TZ99].

\section{KEOPS Broadcast-and-Select Switch}

Most broadcast-and-select switches use WDM technology for switching. A basic example of this kind of switch is the broadcast-and-select switch implemented within the KEOPS project. This switch is illustrated in Figure 4.14 and is also described in Chapter 5, Recent Research Projects in the Area of Optical Packet Switching. As can be seen from Figure 4.14 there are some similarities between this switch and the wavelength routing ones. The difference between packet encoder in this switch and packet encoder in wavelength routed switch in Figure 4.9 is that in this switch the wavelengths given to the packets correspond to the input ports while in the wavelength routing switch wavelengths given to the packets 


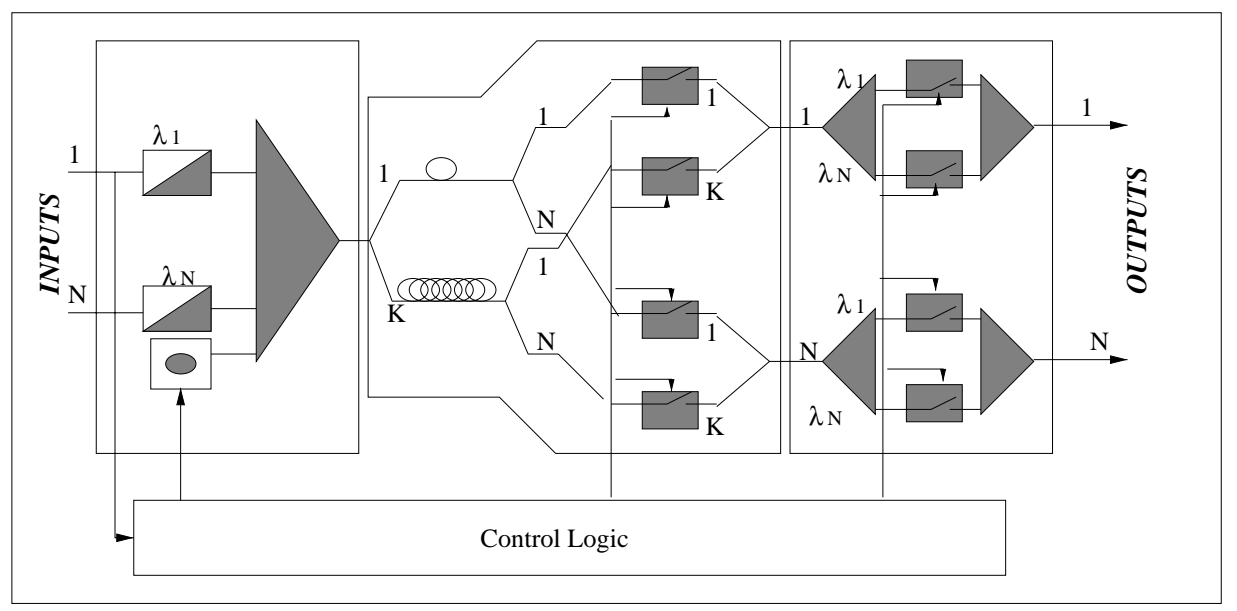

Figure 4.14: The structure of broadcast and select switch implemented in KEOPS project, adopted from $\left[\mathrm{CLJ}^{+} 98\right]$

correspond to output ports. In the KEOPS broadcast-and-select switch each input and output can contain only one packet at a time. An $N \times N$ broadcast and select switch consists of four blocks $\left[\mathrm{CLJ}^{+}\right.$98]:

- Packet encoder consists of $N$ wavelength converters, one for each input. Every input has a specific, fixed wavelength. This means that the input of each packet is identified by the wavelength. All the packets arrived at the same time slot are multiplexed.

- Packet Buffer includes delay lines and a space switching matrix that consists of passive couplers and optical SOA gates. Multiplexed signal is copied to $K$ different delay lines. At every time slot a copy of each delay line's output is sent to all $N$ blocks of the space switch. At each block one of the signals is chosen and the others are dropped. The chosen signal that contains all the packets that arrived at the switch at a specific time slot is then directed to the next block.

- Wavelength selector block consists of $N$ wavelength selectors. Every wavelength selector gets a signal consisting of $K$ packets. The desired wavelength (input port) is chosen according to electronic controller and the desired packet is directed to the output port.

- Control Electronics controls the system. Electronic FIFO buffers are used for storing the relevant information from packet headers. For further information, see [YMD00a, XPR01, CLJ ${ }^{+}$98].

\section{ULPHA Broadcast-and-Select Switch}

Most optical packet switches use WDM rather than OTDM. WDM has many good characteristics, but it has also problems. WDM-based networks need sensitive tunable filters and wavelength converters. Additionally complex electronic control devices are needed. Therefore, a TDM based broadcast-and-select switch 
was implemented by Shimatsu and Tsukada in the early 1990's [ST92]. The switch called ULPHA is an ultrafast photonic ATM switch based on broadcastand-select principle. It is illustrated in Figure 4.15. Input signals are guided to input modulators $(\mathrm{M})$ and cell coders $(\mathrm{CC})$, which compress the data according to the rate of the short pulse source. The header is not time-compressed, which makes it easier to detect the address information. Star coupler combines the data from different inputs into one signal, which is then copied to all outputs. Cell selector chooses right time slot and the packet is then decoded to the original form. As in most switches considered, each input and output port can contain only one packet at time.

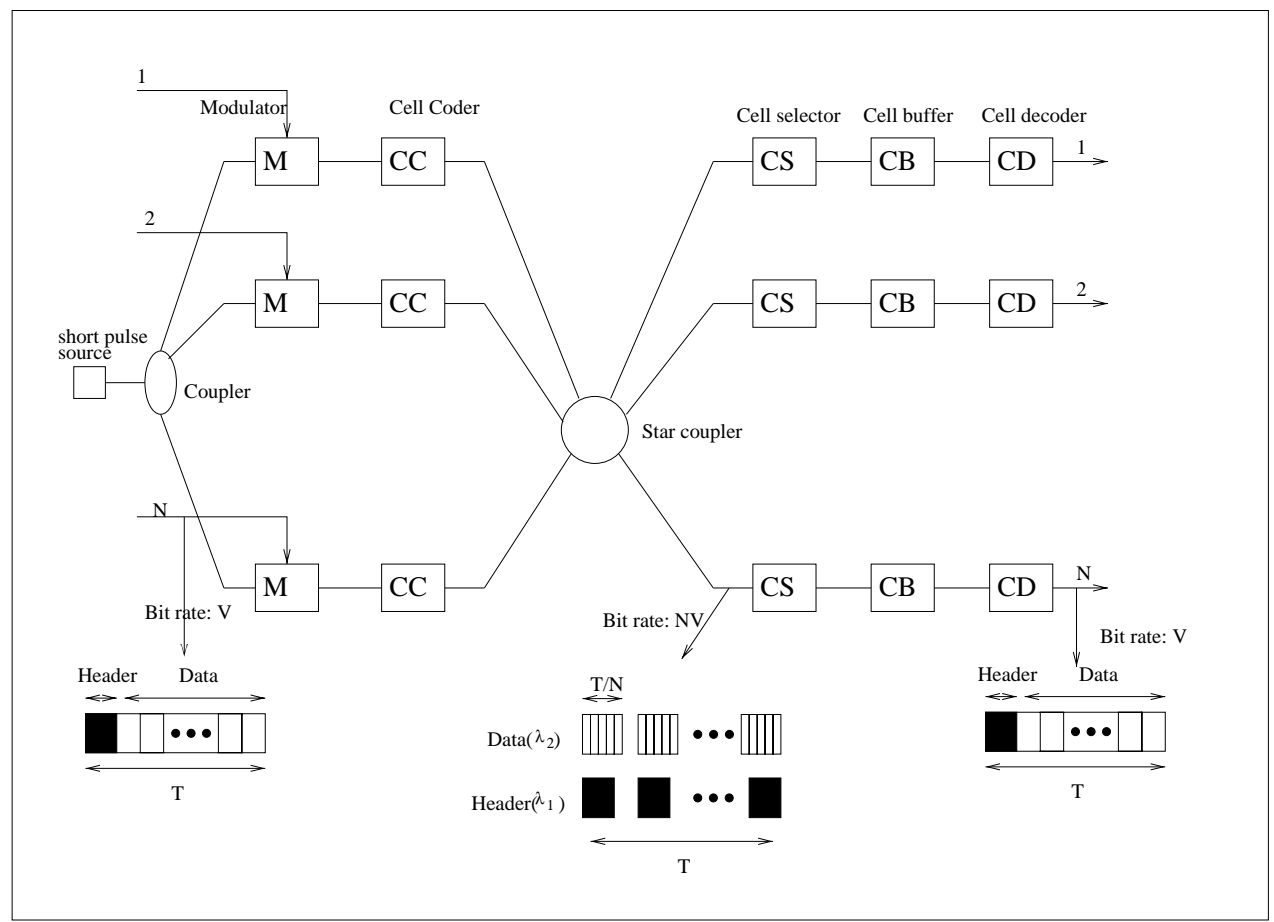

Figure 4.15: The structure of the ULPHA switch, adopted from [TZ99]

\section{Fiberloop Memory Switch}

Fiber loop memory switch illustrated in Figure 4.16 is a simple broadcast-andselect switch implemented in the ATMOS project. It has one TWC at each input and a tunable filter at each output. The switch has a random wavelength accessible recirculating loop buffer. With tunable wavelength converters each packet is given a wavelength that is not in use in the buffer. At every time slot all the packets are copied to all the outputs. The packets that have been chosen by the tunable filters are removed from the buffer with tunable gate switches. The capacity of the switch can be increased by using several buffers and space switches. Again, each input and output port can contain only one packet at time. 


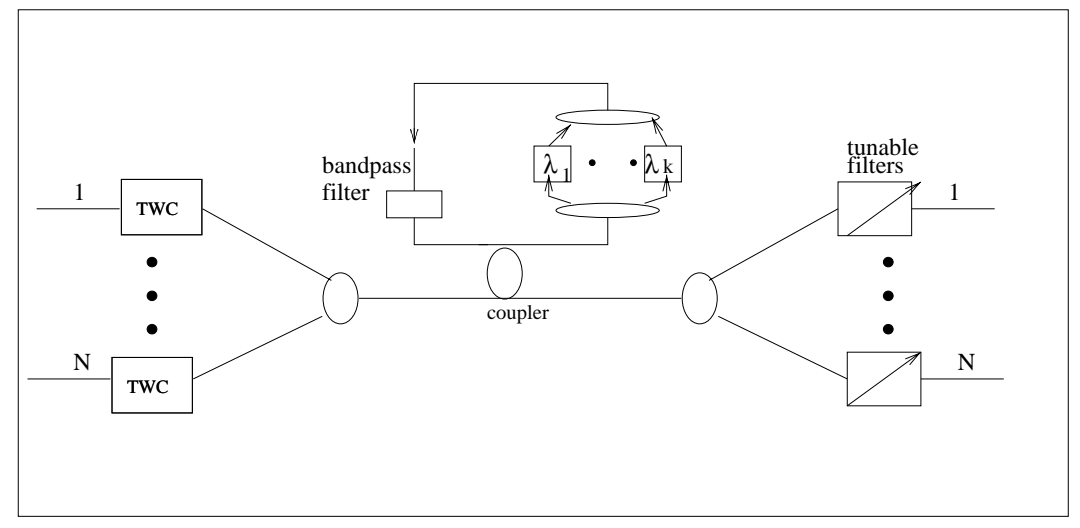

Figure 4.16: The structure of the fiber loop memory switch, adopted from [TZ99]

\subsubsection{Switches in Slotted Networks}

\section{Photonic Slot Routing}

Photonic slot routing is an alternative packet switching scheme. In photonic slot routing time is divided into slots of fixed length. Each wavelength can contain one packet at each slot. The same slot from every channel is destined to same node and therefore each slot can be switched as an integrated unit without multiplexing and demultiplexing [ZJM00]. One wavelength can be allocated for slot headers. Research related to photonic slot routing has mainly concerned ring shaped networks. Figure 4.17 illustrates a switch used in photonic slot routing networks.

First channels are demultiplexed and channels containing header information are directed to the header processing unit. Instead of demultiplexers add-drop filters could be used. Other channels are delayed while headers are processed. Switching is then performed as in normal packet switches with one channel. Finally packets that are to be transmitted are added to a free channel. Compared to switches proposed so far, this switch is simple and less components are needed.

\section{FLAMINGO Project}

A network quite similar to photonic slot networks discussed above was implemented in the FLAMINGO project, which is described in more detail in Chapter 5. FLAMINGO MON is a ring shaped network, where time is divided into slots. Two different networks were implemented. In the first network time is divided into fixed slots, while in the other network there are slots of three different lengths. Unlike in photonic slot routing networks, packets at same time slot at different wavelengths can be destined to other nodes. However, each slot has a common header that is carried in a separate channel.

The network, in which fixed time slots are used, is illustrated in Figure 4.18. Every node is capable of receiving information at all wavelengths but can trans- 


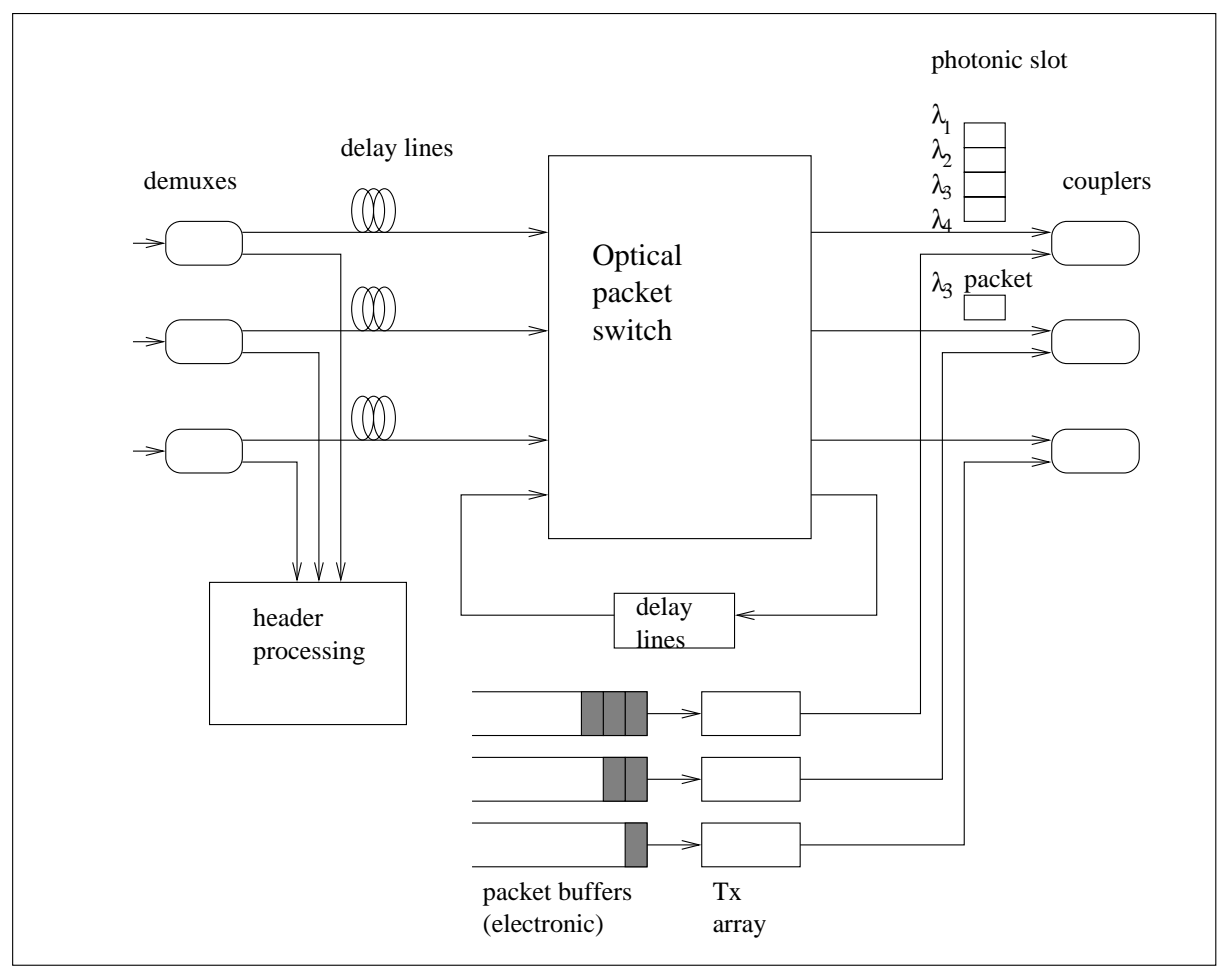

Figure 4.17: The structure of photonic slot routing switch, adopted from [ZJM00]

mit with only one specific wavelength. The first $\lambda$-drop device separates the channel containing header information. Then data channels are delayed the time corresponding the header processing time. Second $\lambda$-drop device separates the channel that is used for transmitting information. The other data channels are demultiplexed. $10 \%$ of signal power is directed to the Rx component and the rest is multiplexed and forwarded to $\lambda$-add devices. Data in the Rx component is either destroyed or used according to the header information. First the channel with transmitted information is added and then the header channel is added with the other device. Finally signal is amplified and forwarded.

All the above is performed optically. While the data is in delay line buffer, the header channel is directed to header processing block. According to the header information the switch $\mathrm{A}$ is in one of the possible states. It can be in the bar state, if the information in a specific channel (at this time slot) is not meant for this node. It can be in the cross state if the information is for this node only, or it can be in the split state is the information is meant for this node and other nodes. If the switch gets bar state, the part of the header slot related to the studied channel is not changed and the corresponding data slots in Rx components below are discarded. If the switch is in cross or split state the information in the $\mathrm{Rx}$ component corresponding to the channel studied is not discarded but forwarded out of the network. In split state the part of header slot related to the channel in question is not changed, but if the switch is in cross state the header information forwarded to next node is changed, i.e., the channel is marked unused. Note that the content of data channels is not changed in any manner. The content of each 


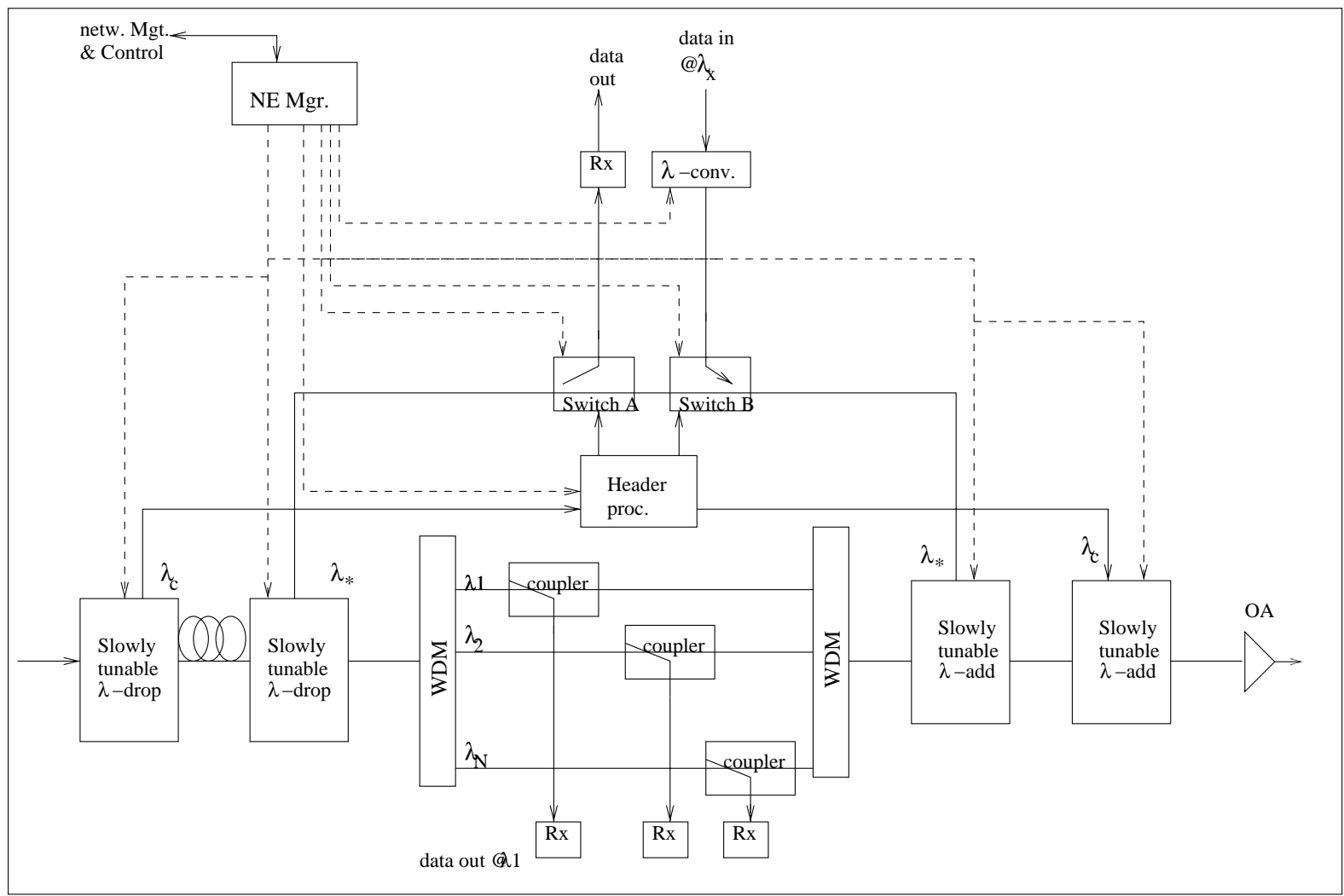

Figure 4.18: A switch in network with fixed time slots, adopted from [SdGD01]

data channel can be changed only in the node which transmits with that channel. As explained above, the transmission channel is separated from the others. Its header information is studied in the header processor. If a time slot is marked empty or it would be marked empty at this node, new data can be converted to wavelength of this channel and a packet can be added to the right slot by switch B. If header information tells that the data slot is in use, the slot is forwarded with its current content. If the transmission channel contains information for this node, it is retrieved from the component Rx.

The other alternative is to build a network with varying slot size. In the network proposed in [DKGS01, DvBK $\left.{ }^{+} 01\right]$, there are slot sizes of three different lengths. The switch in the flamingo network, i.e., an access point (AP), is illustrated in Figure 4.19. The tunable $\lambda$-drop separates the control channel containing the header information. The $\lambda$-drop device could be fixed, but tunable device is more secure, because if the channel allocated to header information breaks and there is no alternative channel, the whole system fails. Control channel's content is directed to electronic header processing unit (HPU). Data channels are guided to delay line corresponding to the header processing delay. The delay is controlled with the header processing unit. The delayed data slots are then demultiplexed. If the slot is empty a new packet can be added to that slot. If there is a packet in the slot it is either switched to the next link or, if this is its destination AP in this network, it is removed from the net and guided to other network. After the 


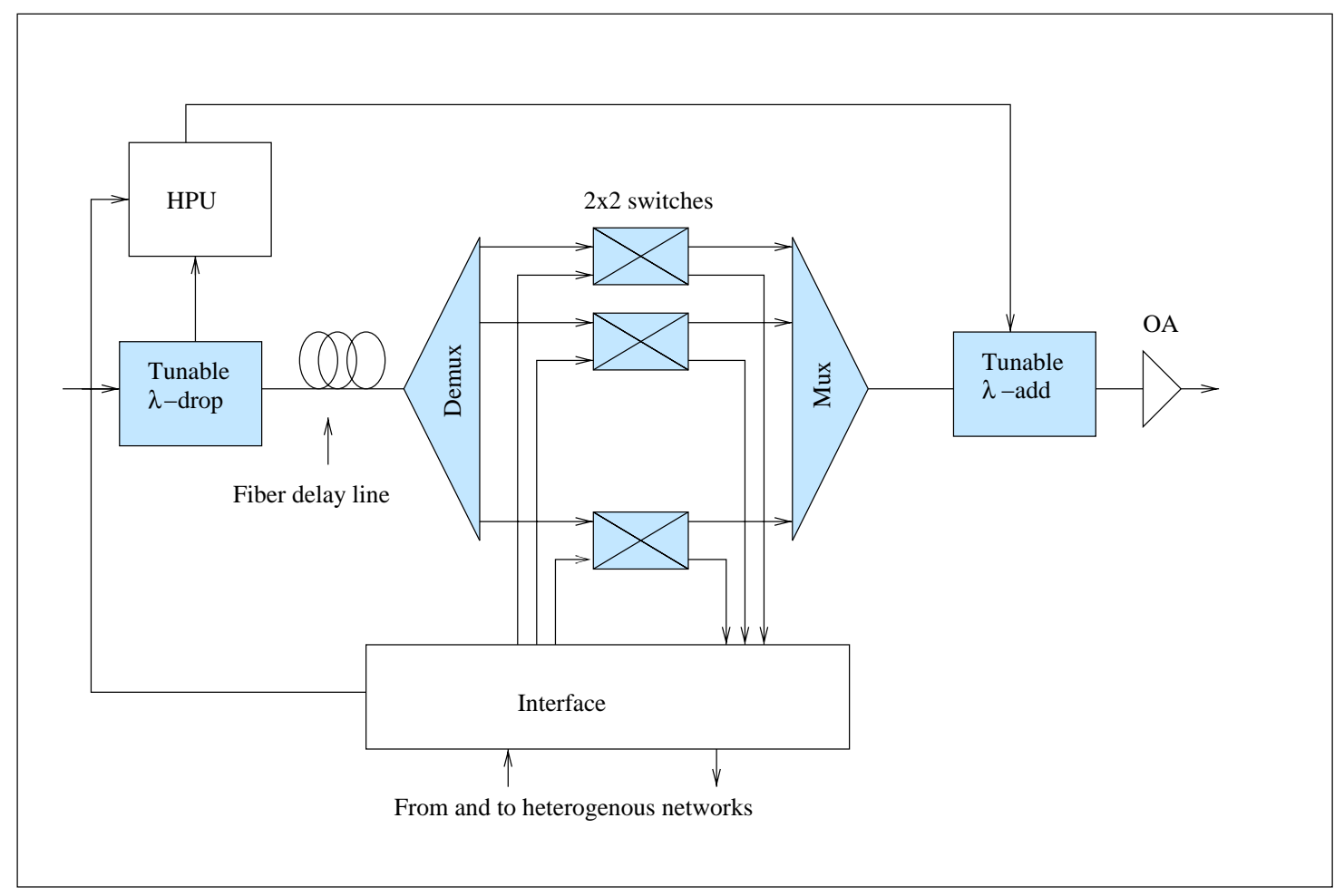

Figure 4.19: Access point in FLAMINGO MON, adopted from $\left[\mathrm{DvBK}^{+} 01\right]$

switching is performed the packets are multiplexed and updated header channel is included in tunable $\lambda$-add device.

\subsubsection{Comparison and Conclusions}

Many switch types illustrated were capable of switching only one packet from each input at time. These switches could not be used in WDM networks without modification. In todays commercial systems the number of channels is 40 or 80 . This number will probably increase in the near future. Therefore the performance these switches is remarkably weaker than of those that can be used in WDM networks.

One of the switches capable to WDM networks was the WASPNET switch that actually was composed of $N$ identical 1-wavelength switches. The number of components needed in WASPNET switch is high. Another of these switches was space-switching-based bufferless switch illustrated in Figure 4.6. This switch type could be also modified by replacing tunable wavelength converters with buffers. The switch designed in the DAVID project is also capable of switching several packets from each input at the same time.

The other type of switches capable to WDM networks are the photonic slot routing based switches. These are meant for WDM networks. They are not as flexible as switches in normal optical networks because channels are not used 
individually but as part of a slot. However, they use the capacity of the fiber almost as efficiently as normal WDM based packet switches. On the other hand the number of components needed is something like the number of components needed in 1-wavelength switches. Additionally, these switches are easier to manage.

In all switches studied here except in one of the FLAMINGO project switches and in the switch studied in the DAVID project, the packet size is fixed and networks are slotted. In the FLAMINGO network time slots, that is, packets, can be of three different lengths. In the DAVID project the packet length is truly variable. Switches in slotted networks are easier to manage, which make them attractive. On the other hand, switches with variable length packets can handle the bursty data traffic better.

As a conclusion it can be said that most optical packet switches use both wavelength conversion and optical buffers for contention resolution. Most switches proposed have fixed length packets and they are meant for slotted networks. Some approaches use variable length packets, which have certain benefits. However, switches in slotted networks, especially photonic slot routing switches, have better possibilities to be used outside the laboratories earlier, because they are less complex. Switching speed is one of the main problems, and therefore optical burst switches, that are discussed in subsequent chapters might be a solution to many problems.

\subsection{Contention Resolution}

\subsubsection{Space Deflection}

Because optical buffering has several problems, it has been avoided as much as possible. Deflection routing, also known as hot potato routing, is an alternative where no buffers are implemented. If contention occurs and a packet cannot be switched to the right output, it is routed to any other output instead. If one is lucky, the packet finds another way to the destination node. This works quite well in small networks with high connectivity, i.e., if the nodes have several neighbors. If the network has low connectivity, it is more probable that a deflected packet will not reach the destination node. Because this packet spends resources by traveling in the network without finding the destination node, it is clear that other contention resolution schemes are better in this situation. Additionally, deflection routing can be only used in networks with low load. If average traffic load is high, deflected packets will only decrease the efficiency of the network. Deflection routing can be improved by accepting only certain ports, i.e., if a packet cannot find a reasonable way to the destination it is blocked although there were free outputs [YYMD00]. 


\subsubsection{Optical Buffering}

Switching in time domain in optical systems is problematic due to the lack of suitable optical RAM. Although the subject has been investigated, no feasible and useful optical RAM has been implemented [TZ99]. The main difference between electronic and optical time switches is the ability of electronic memories to store a packet for an indeterminate time. An optical buffer consists of delay lines. When a packet is guided to a delay line, it is not possible to take it back before it has reached the other end of the line. Another problem of optical buffers is the power loss signal suffers when guided through a delay line [YMD00a]. To compensate the power loss, either amplifiers or signal regenerators have to be used. The former alternative leads to noise and the latter is expensive.

There are two classes of optical delay line buffers: recirculating type and traveling type [TZ99]. Additionally traveling type delay lines could be divided into two classes: normal delay lines and cascaded systems that contain several sets of delay lines. The recirculating type offers the possibility to use same delay line several times, i.e., to recirculate packet. The problem with the recirculating type is the power loss that leads to the need for amplification and therefore also to noise. This limits the maximum buffering time or leads to the need for regeneration between the buffering times.

In the traveling type buffers the time packet is buffered is determined totally beforehand; a delay line cannot be used several times. Of course, if there are several delay line groups serially connected, the delay can be determined in several pieces, but the length of the delay lines determines the maximum delay anyway. An example of a cascaded delay line system implemented is optical synchronizer in KEOPS project [CLJ ${ }^{+}$98]. The synchronizer is illustrated in Chapter 5. It consists of three cascaded delay line sets that each include four delay lines and four optical gates. First delay line set includes lengths 0, 8, 16 and 32, the next contains lengths $0,2,4$ and 8, the last set contains lengths $0,1,2$ and 3. This way more accurate delays can be implemented without several recirculations. On the other hand, extra components (optical gate for each delay line) are needed.

\subsubsection{Wavelength Conversion}

Optical networks have an additional dimension, the wavelength. If contention occurs in a system using wavelength conversion, one packet is passed through and the other is guided to the same output port but with a different wavelength. This solution is optimal in the respect that neither packet is delayed. This approach is more suitable for circuit switching, because a need for fast tunable wavelength converters restricts the use in optical packet switching networks. However, recent research results have shown that it is a promising alternative also in packet switched networks. 


\subsubsection{Performance of Different Contention Resolution Schemes}

The most effective combination of these is to use space deflection, buffering and wavelength conversion together. If cheap solution is needed, then minimum optical buffering with space deflection is the solution [YMD00b, YYMD00]. 


\section{Chapter 5}

\section{Recent Research Projects in Optical Packet Switching}

\section{$5.1 \quad$ ATMOS}

ATMOS (ATM Optical Switching) project was accomplished in the second stage of the RACE (Research and Technology Development in Advanced Communications in Europe) program. It concentrated on studying possible photonic technologies for high speed ATM switching systems [FMH97]. Partners contributing to this project were Alcatel Alsthom Recherche, Alcatel CIT, Alcatel SEL, Ascom Tech, BT, Centro Studi e Laboratori Telecom (CSELT), Eidgenossische Technische Hochschule Zürich, France Telecom CNET, IMEC, Technical University of Denmark and Thomson CSF. The objective was to build and test novel switches and switching systems. Also optimization of key optical components was studied. Optical delay lines where first time studied in Europe [CFK $\left.{ }^{+} 96\right]$. In switches built during this project both electronics and optics were used. Space switches and wavelength routing were studied and also optical delay lines were tested. Performance of optical switches was studied theoretically, optical ATM switch $(4 \times 4$ at 2,5 Gbit/s with fiber delay lines) was demonstrated, and a few components including tunable filters were built. For further information, see [Ben97].

One of the achievements in the project was implementation of a simple broadcastand-select switch called fiber loop memory switch. The switch is described in more detail in Chapter 4. For further information and simulation results, see also [KBS01].

\subsection{KEOPS}

The KEOPS (KEys to Optical Packet Switching) project was a three year project from September 1995 to September 1998 performed by ACTS (Advanced Communications Technologies and Services). Participants contributing to KEOPS were partly the same as those in ATMOS: Alcatel Alsthom Recherche, Alcatel CIT, Alcatel SEL, France Telecom, CSELT, Technical University of Denmark, Uni- 
versity of Bologna, ETH Zürich and University of Strachlyde [RMGB97]. The project was based on the outcome of the ATMOS project. KEOPS project is still one of the most important projects in the area of optical packet switching, and many relevant results were obtained in the project. For instance, the switch models created within the project have been base structure for optical packet switches built within other projects. The main objective of the KEOPS project was, according to its final report, "definition, development and assessment of optical packet switching and routing networks capable of providing transparency to the payload bit rate, using optical packets of fixed duration and low bit rate headers to facilitate processing at the network/node interfaces" [CJR ${ }^{+} 98$ ].

\subsubsection{Components}

During the project all-optical wavelength converters with conversion rate $10 \mathrm{Gbit} / \mathrm{s}$ were developed to relatively maturity. The wavelength converters developed are based on cross-phase modulation in SOAs placed in interferometric structures. These devices were also used as the core of all-optical regenerators implemented during the project. The work has been later continued within ACTS REPEAT and PELICAN projects [LDA $\left.{ }^{+} 99\right]$. Also clamped-gain semiconductor optical amplifier gates, tunable/multiwavelength sources and fast wavelength selectors were implemented. Two important devices implemented in the project are an alloptical synchronizer and a $3 \mathrm{R}$ regenerator $\left[\mathrm{CLJ}^{+} 98\right]$.

\section{Synchronizer}

Synchronization of packets is one of the problems in optical packet networks. During the KEOPS project an all-optical synchronizer, illustrated in Figure 5.1 was implemented. The synchronizer consists of three cascaded stages each of which contains two passive couplers, four delay lines and four optical gates [CLJ ${ }^{+}$98]. The first set of delay lines with coarse accuracy removes remarkable differences in arriving times while the two other blocks are for fine adjustment. Each packet is compared to reference value and according to that optical gates are switched on or off. The resolution of synchronizer implemented is about 3.2 ns and operation time is less that $1 \mathrm{~ns}\left[\mathrm{CLJ}^{+} 98\right]$. Signal to noise ratio and interference between different paths are low.

\section{R Regenerator}

Optical regenerators are divided in three classes: $1 \mathrm{R}$ regenerators are capable of amplification, $2 \mathrm{R}$ regenerators can both amplify and reshape signal, and $3 \mathrm{R}$ regenerators can amplify, reshape and re-time signal $\left[\mathrm{LDA}^{+} 99\right]$. The function of the $3 \mathrm{R}$ regenerator implemented in the KEOPS project is illustrated in Figure 5.2.

As many other components of optical packet switches, the $3 \mathrm{R}$ regenerator uses SOA technology. First an arriving signal is directed to a SOA based wavelength converter that uses cross-gain modulation. Its function is to decrease influence of power variations and to help to achieve polarization and wavelength insensitivity. Next, the signal is guided to the sampling stage that performs regeneration 


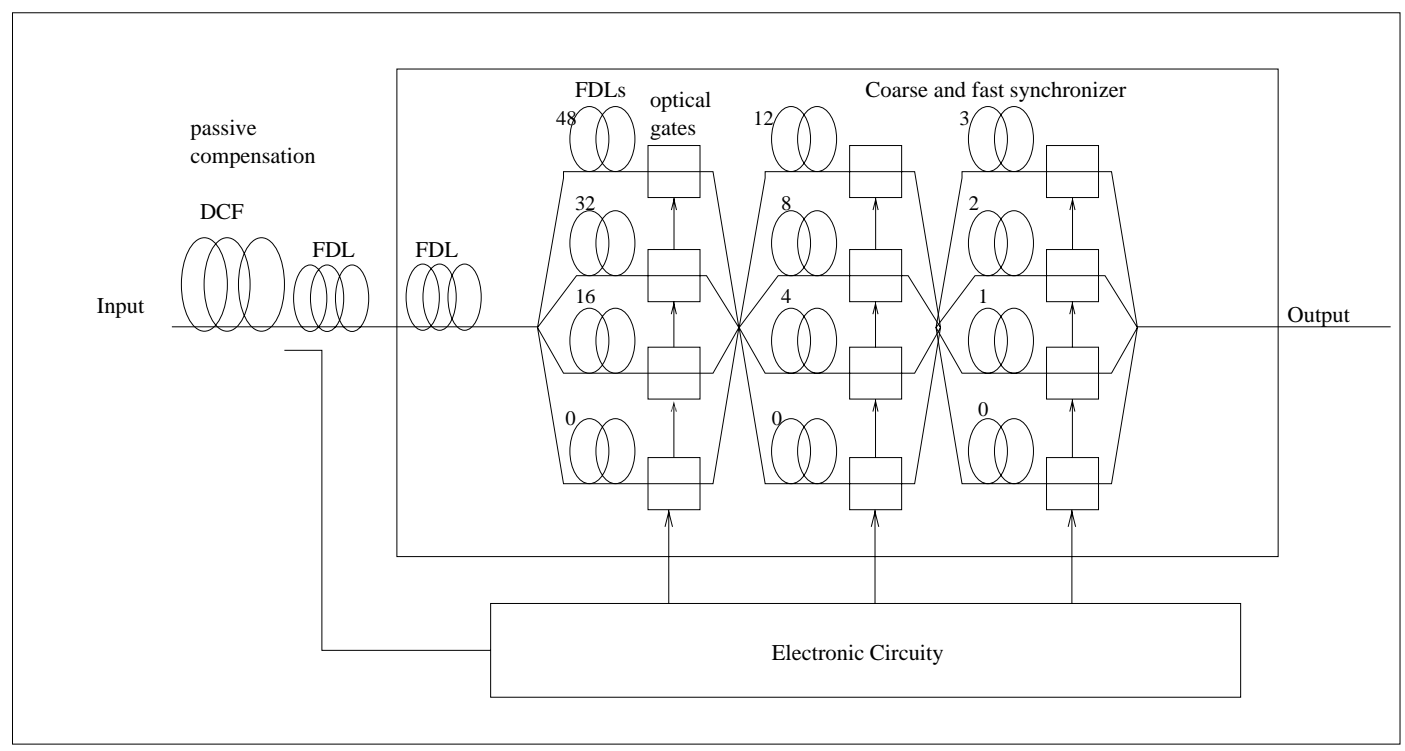

Figure 5.1: Structure of the all-optical synchronizer, adopted from [CLJ $\left.{ }^{+98}\right]$

in time domain. The sampling stage consists of an external modulator, a clock recovery circuit, EDFA and a wavelength selector. Then, the signal is directed to the second SOA-based wavelength converter. Unlike the other, this wavelength converter is based on cross-phase modulation. This component is used as a decision gate to reshape the signal in the amplitude domain. The update of the header is also performed here. At the output port the signal is guided through an EDFA and a bandpass filter.

\subsubsection{Switch Architectures}

In the KEOPS project two switching fabrics, wavelength routing switch and broadcast-and-select switch were implemented. They both were discussed also in Chapter 4. Also the SLOB switch(Switch with Large Optical Buffers) was designed partly in connection with the project.

\section{Wavelength Routing Switch (WRS)}

As the name of the switch suggests, WDM is used for switching in wavelength routing switch. Figure 5.3 illustrates the architecture of the switch. The network demonstrator implemented during the project consists of a $4 \times 4$ switch with 4 wavelengths and payload signals of $2.5 \mathrm{Gbit} / \mathrm{s}$. The demonstrator included contention resolution with optical delay lines, payload routing according to the input header content, header updating in the switch, and rewriting with respect to the payload position.

Implementing a wavelength routing switch is expensive. To get an idea of the complexity of the switch, components needed are listed below. Because core 


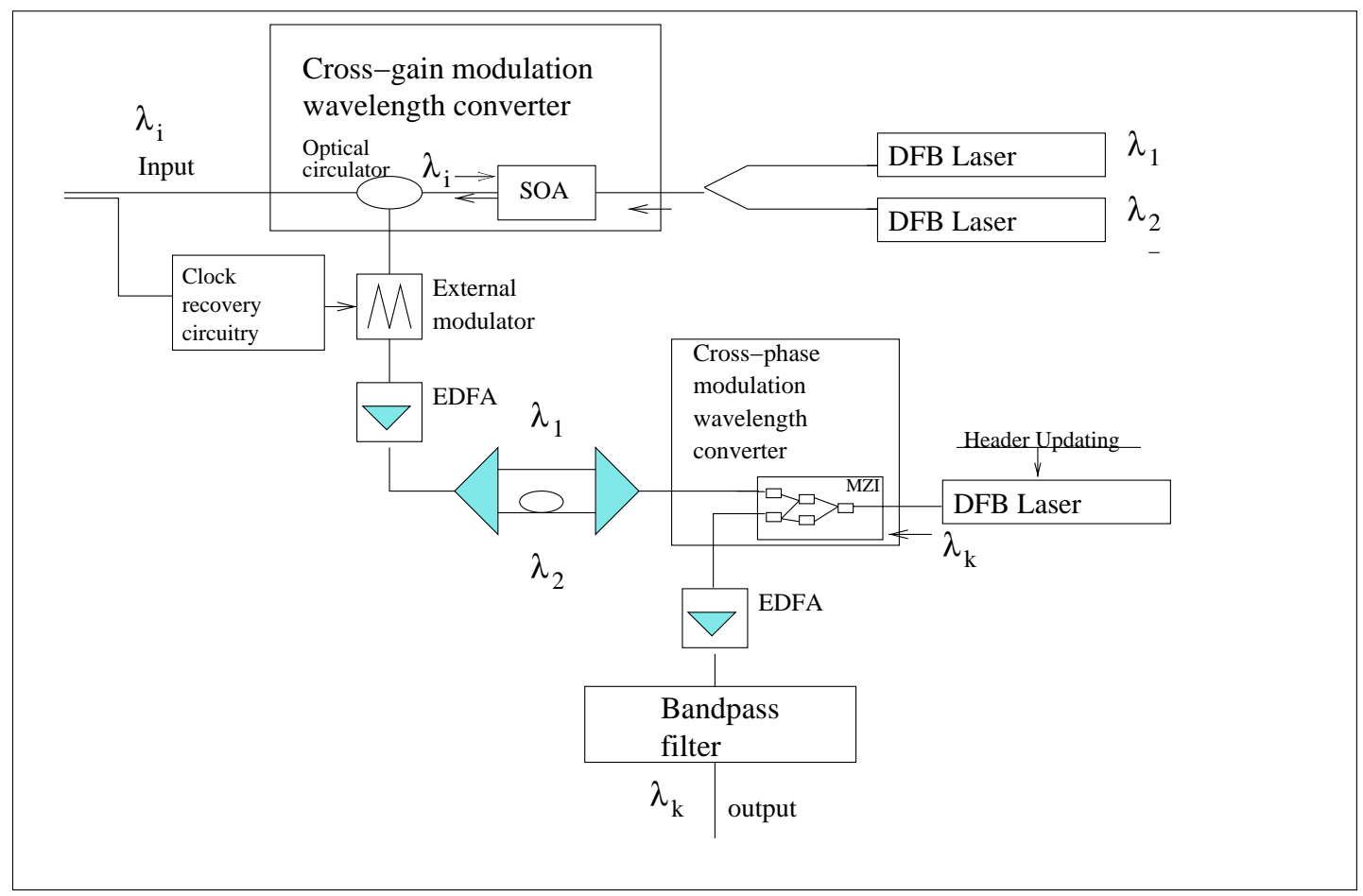

Figure 5.2: The function of the $3 \mathrm{R}$ regenerator, adopted from [CLJ $\left.{ }^{+} 98\right]$

and edge nodes need different number of components, the average number of components needed in different nodes is given: 1.5 Mach-Zehnder Interferometers, 1.5 SOA wavelength converters, 80 DFB lasers, 3 EDFA, 3 notch filter, 12 de/multiplexers and $16.8 \mathrm{~km}$ delay line [CJR $\left.{ }^{+} 98\right]$.

\section{Broadcast-and-Select Switch}

A broadcast-and-select switch is illustrated in Figure 5.4. The switch implemented in the test-bed was a $16 \times 16$ switch with 16 wavelengths and 16 memory positions and $10 \mathrm{Gbit} / \mathrm{s}$ signals [CJR ${ }^{+} 98$ ], while the switch illustrated in Figure 5.4 is a more general $N \times \mathrm{N}$ switch with $K$ memory positions. The test-bed results for the broadcast-and-select switch were promising. The experimental validation of the KEOPS concept at the network level was successful [CJR ${ }^{+}$98]. The drawback of the architecture is the need for a great number of components [TZ99].

\subsubsection{Packet Format}

A packet format fixed in time but not in bits was used in the KEOPS project. The amount of data per packet can be freely chosen as long as the duration of the packet remains fixed. The header of the packet is encoded with relatively low (hundreds of Mbit/s), fixed bit rate and the rate of the payload is proportional to the user defined bit rate, which has to be between $622 \mathrm{Mbit} / \mathrm{s}$ and 10Gbit/s. Because the transmission rate of the header differs from the data rate of the payload, it is possible to use switching speed slow enough and obtain good throughput at 


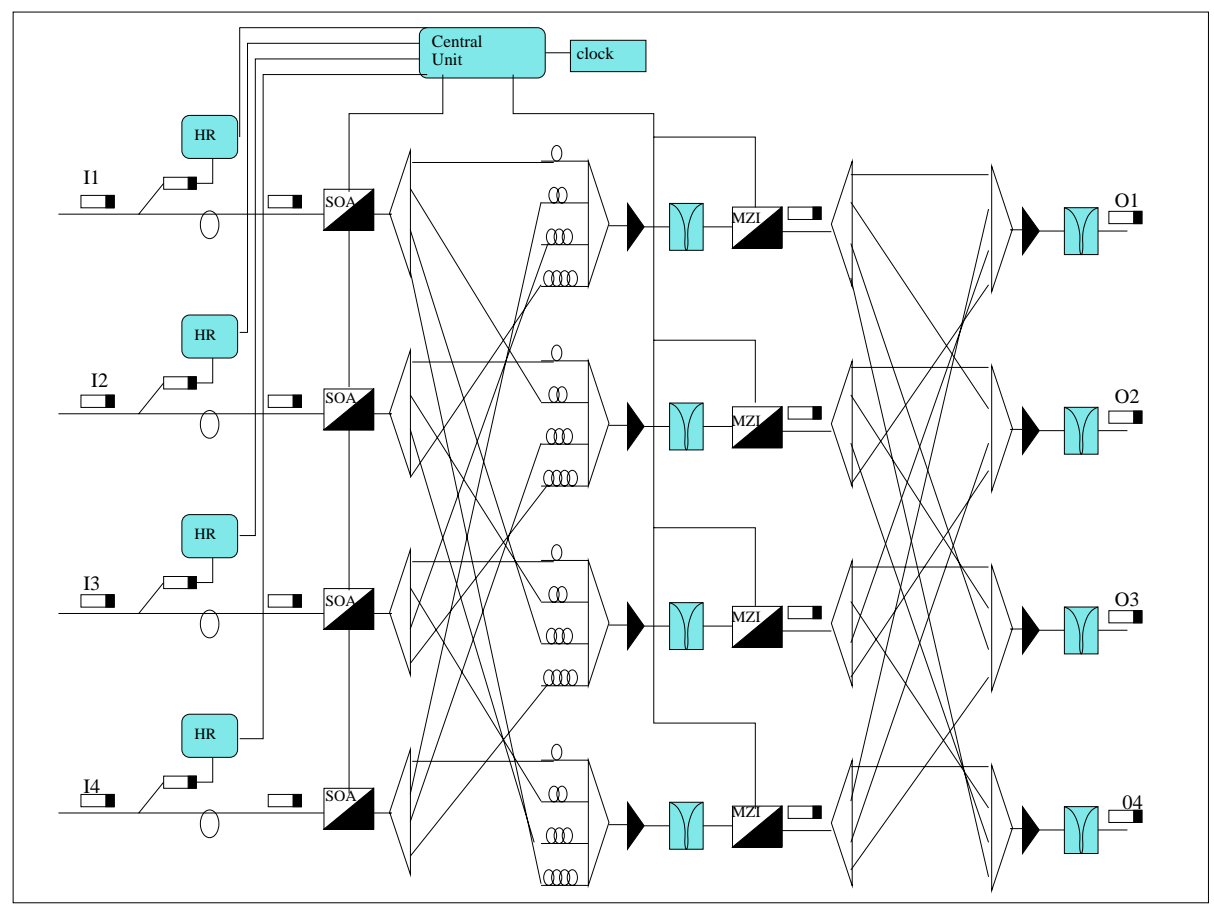

Figure 5.3: Architecture of Wavelength Routing Switch (wrs), adopted from [CJR ${ }^{+} 98$ ]

the same time. Figure 5.5 describes the packet structure [CJR ${ }^{+} 98, \mathrm{CC} 97$ ].

The structure of the header is the following. 8 bytes are dedicated to hierarchy of routing labels and 3 bytes are reserved for identification of payload type, flow control, packet numbering etc. 1 byte flags the position of the payload relative to the header. 2 bytes were reserved to header synchronization pattern. Total length of the header is therefore 14 bytes [CJR $\left.{ }^{+} 98\right]$.

Between the header and the payload there is a gap, the guard time, which ensures that jitter will not cause any problems and the payload can be transmitted to the destination undamaged. There is a guard time before and after the payload. The duration of the guard time varies, and consists of two parts. The first part is fixed time, which is always waited. Additionally, there is a second part, which is proportional to the time slot duration. The optimal payload duration analyzed for ATM traffic is $2.726 \mu \mathrm{s}$. The value is based on the transmission efficiency and, on the other hand, the fact that the length of the delay line buffers used for buffering the packets is limited.

\subsection{WASPNET}

WASPNET (Wavelength Switched Packet Network) is an EPSRC funded collaboration between three British universities and several industrial institutions. The academic participants are University of Strathclyde, Essex University and Bristol University and industrial institutions include BT Laboratories, Marconi Commu- 


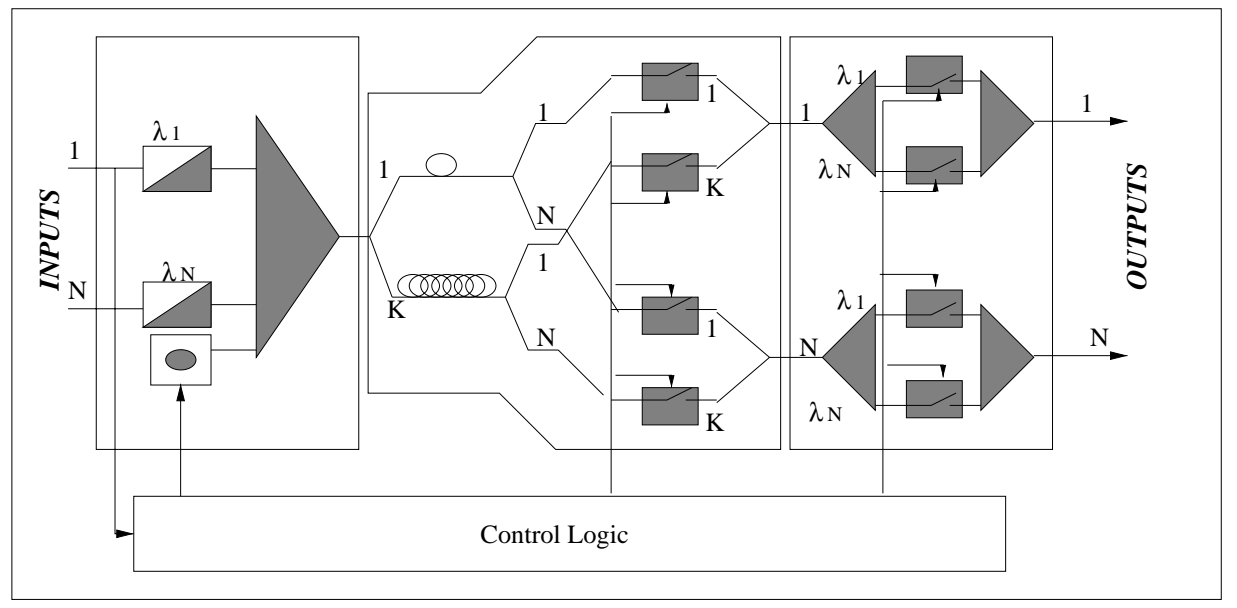

Figure 5.4: Architecture of Broadcast-and-Select Switch, adopted from [CJR ${ }^{+}$98]

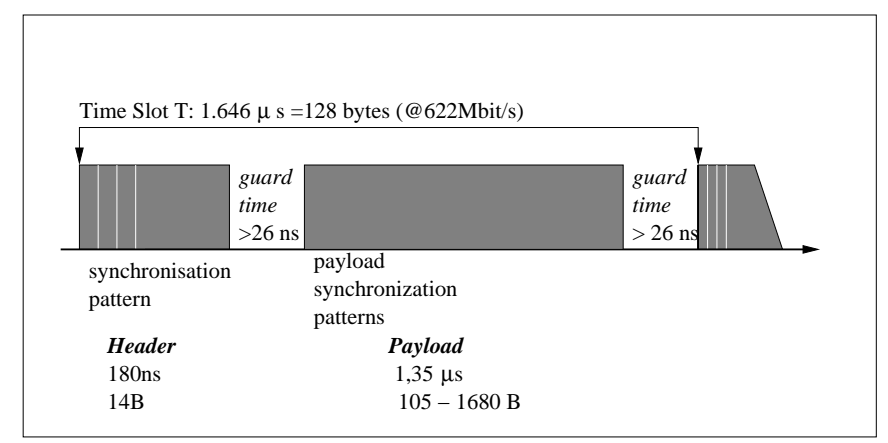

Figure 5.5: KEOPS packet format, adopted from [CJR ${ }^{+98}$ ]

nications, and Fujitsu Telecommunications. The project started in July 1997. The objective of the project is to study a novel packet based optical transport network $\left[\mathrm{HNC}^{+} 99\right]$.

One of the main achievements of the project is the implementation of a few optical packet switch architectures. High capacity switching, compatibility with Internet traffic and efficient use of the network have been obtained as a result of the project. It was also shown by WASPNET project that optical packet transmission in a network of over 14 nodes is possible without significant signal degradation. In addition to node design, also network control and operation and device fabrication were studied. During the project a network demonstrator has been developed. Using the demonstrator the functions of header deletion and insertion, routing and switching and contention resolution have been studied. For further information, see $\left[\mathrm{HNC}^{+} 99, \mathrm{CHA}^{+} 01\right]$.

\subsubsection{The WASPNET Switch}

The switch illustrated in Figure 5.6 is a simplification of the WASPNET WDM optical packet switch. The figure illustrates one plane of the switch while the 


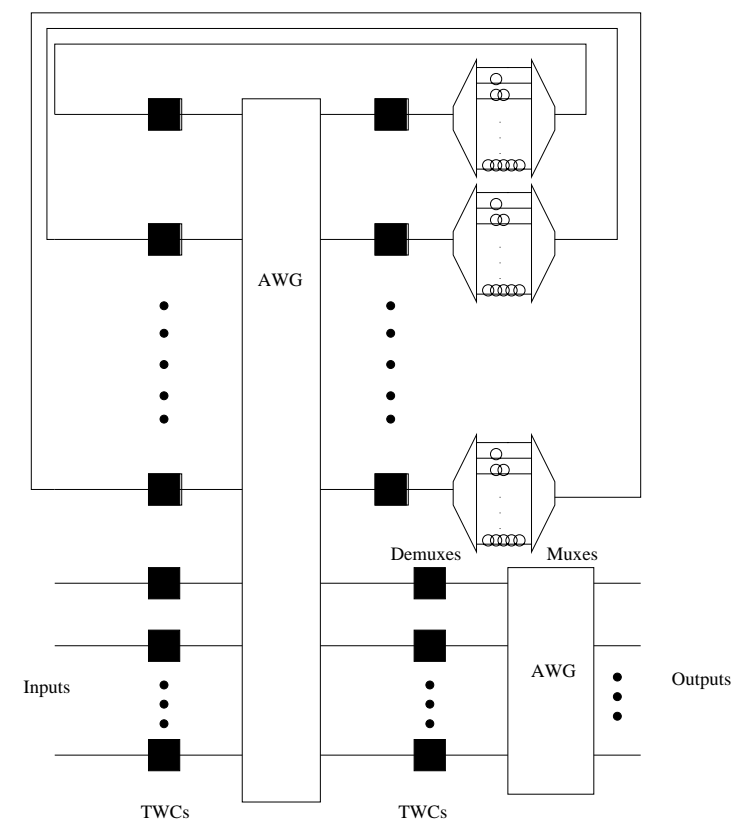

Figure 5.6: WASPNET switch, adopted from $\left[\mathrm{HNC}^{+} 99\right]$

switch consists of multiple planes each operating at a single wavelength. In WDM capable WASPNET switch each input fiber is connected to a demultiplexer that sends packets with wavelength $i$ to plane $i$, and each output is obtained by multiplexing a combination of the outputs of the planes. In this switch, higher priority packets can preempt low priority packets. The function of the switch is mainly similar to the function of the AWG-based feed-back switch. The second AWG in the figure is needed to avoid contention. The combination of wavelength and input port of the second AWG determines the output port. Multiple packets may exit on the same output of the plane, each at different wavelength. If this has to be eliminated, another alternative is to replace the second AWG with a space switch, as proposed in $\left[\mathrm{CHA}^{+} 01\right]$.

\subsubsection{Network Control}

During the project two possible network control methods were identified: the scattered wavelength path (SCWP) and shared wavelength path (SHWP) multiplexing schemes. In the latter approach each optical packet uses the same wavelength along the whole path. Several packets may use the same wavelength. In the former approach, SCWP, packets can be converted to a new wavelength at each node. Naturally, SCWP offers higher throughput and demands less buffers. On the other hand, a number of fast tunable wavelength converters are needed. For further information, see $\left[\mathrm{HNC}^{+} 99\right]$. 


\subsubsection{Packet Format}

In the WASPNET project, a packet with 4 bytes long header and 256 bytes long payload were used. The payload had data rate $10 \mathrm{Gbit} / \mathrm{s}$, while the header was sent with relatively low transmission rate. Three different alternatives to transmit the header and the data, each of which has its strong and weak sides, were studied:

- Both header and the data were transmitted at the same time slot. The header was placed on an electrical subcarrier above the frequencies used by the payload.

- The header and the payload were transmitted simultaneously with different wavelengths.

- The header and the payload were transmitted serially, as in the KEOPS project.

The two former schemes suffer from crosstalk. In the first scheme the header updating is difficult, and in the second scheme and additional laser is needed to every payload channel in order to update the header. In the last scheme bandwidth utilization is poorer, but there is no cross talk, and header updating is easier.

\subsection{FLAMINGO}

Flexible Multiwavelength Optical Local Access Network Supporting Broadband Services (FLAMINGO) is a research project supported by Dutch Technology Foundation, STW. Partners participating in the project are Centre for Telematics and Information Technology (CTIT) from University of Twente, MESA Lightwave Devices Group from University of Twente and Photonics Integrated Circuits Group from Delft University of Technology. According to the home page of the project, http://wwwhome.ctit.utwente.nl/flamingo/, the major objective of the project is to investigate promising technological advances in the area of Gbit/s throughput optical telecommunications regarding their capabilities to support broadband multimedia services in optical local access networks. In the project two versions of slotted, ring-shaped, optical packet networks have been proposed.

The FLAMINGO network studied in the project is illustrated in Figure 5.7. FLAMINGO MON is a ring-shaped, slotted WDM network. It consists of access points (APs) connected with fiber links. The access points constitute an interface to a wide area network as well as an interface to local area networks. Access points also control traffic. One link consists of several channels. One wavelength is for header traffic while all the others are for data. Each time a slot in the control channel contains one header packet that consists of the header information of all data packets in all channels at the same time slot. In the FLAMINGO project two switching architectures were proposed. One uses fixed time slots while in the other the channels are divided into payload slot with sizes of 44, 552, and 1500 
bytes $\left[\mathrm{DvBK}^{+} 01, \mathrm{DKS} 00\right]$.

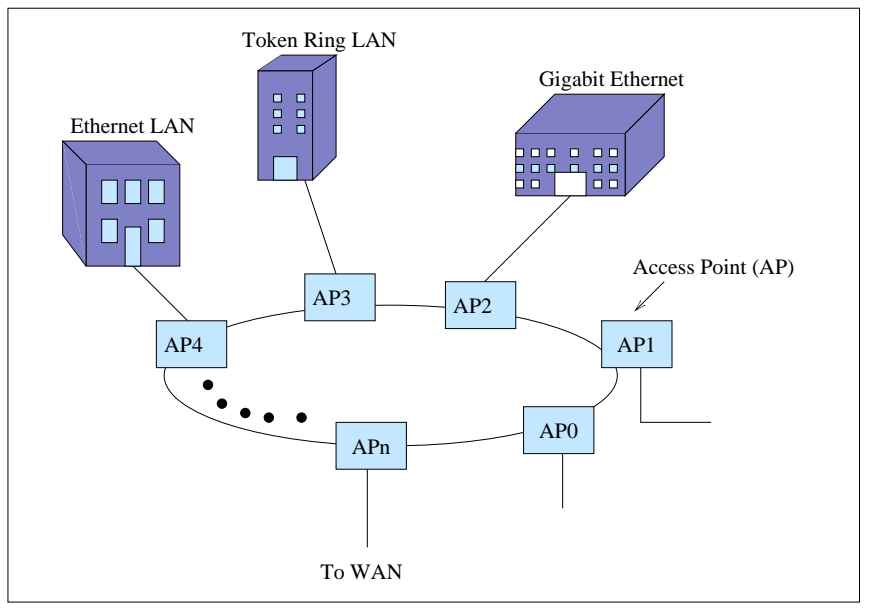

Figure 5.7: FLAMINGO MON, adopted from [DKGS01]

In the network with fixed length time slot every node is capable of receiving information at all wavelengths but can transmit with only one specific wavelength. Therefore one node can have maximum transmission capacity of one channel, even if all the channels are empty.

Main problems of the network with variable size time slots are linked with synchronization. Because of chromatic dispersion the propagation delay in different channels is different. Additionally, in the latter network header slots arrive to access points after substantial amounts of time depending upon the size of payload slots, which leads to loss of clock-synchronization.

\subsection{DAVID}

DAVID is a three year project from July 2000 to July 2003 supported by the EU Commission. 14 partners including companies, operators, universities and research centers are participating in the project. The main objective is to propose a packet-over-WDM network solution, including traffic engineering capabilities and network management, and covering the entire area from MAN to WAN. The project will utilize optics as well as electronics in order to find the optimum mix of technologies for future very-high-capacity networks. More information about the project is available on the home page of the project, http://david.com.dtu.dk.

In the project a model of an optical packet switch has been proposed [CCR02]. Particularly quality of service issues and use of variable length packets have been addressed. The contention resolution is done by combining optical buffers and wavelength division multiplexing. The architecture and operational principles of the switch are explained in Chapter 4. 
Three techniques to support quality of service levels were proposed. In the switch illustrated in Chapter 4 contention resolution is performed with delay lines and wavelength converters. Contention resolution at delay lines and at output ports is avoided by converting packets to different wavelengths, if they are in the same place at the same time. The first technique to support the quality of service classes is based on wavelength allocation. Some wavelength converters can be used by all the classes, some only by the higher classes and the rest only by the highest class. In [CCR02] a model with three classes was studied, but also systems with other number of classes could by easily implemented. The second alternative is a combination of wavelength allocation and threshold dropping. Here only the packets of the highest class have their own wavelength converters. The two lower classes are separated by dropping the packets of the lowest class when the content of the buffer exceeds a certain threshold. The third way is wavelength allocation with scheduling. Wavelength converters are divided into different classes as in the first model. In addition to this, however, repeated wavelength search is used for the higher traffic classes, i.e., if a desired wavelength is not found, and the packet is to be lost, its delay is increased and the search is performed again. For the packets in the highest class the search is done three times, while in the intermediate class it is performed only twice.

\subsection{Other Projects}

In addition to the presented projects many other studies related to optical packet switching have been conducted in the academic community, e.g.,

- British Telecom laboratories demonstrated several aspects of optical packet networks in the late 1990's.

- Photonic Integrated Gigabit Switches (PIGS) was an EPSRC-funded program. The switches implemented in the project consist of several smaller switches switched in serial. The aim was to obtain switches with moderate buffer length, and capability of limited deflection routing $\left[\mathrm{HNC}^{+} 99\right]$.

- VCSEL Based Very High Capacity Photonic Packet Switch (VIVALDI) was an EPSRC funded project from January 1999 to December 2001. It focused on the design issues of opto-electronic switches with optical fan-in and fanout. The ultimate aim was to design an IP router, which could operate overall data rates in the Petabits/sec regime [WCS00]. 


\section{Chapter 6}

\section{Strong and Weak Points of Optical Packet Switching}

\subsection{Unsolved Questions}

Optical buffering and contention resolution are main problems in optical packet networks. These were discussed in Chapter 4. In this chapter other unsolved questions concerning optical packet switches are discussed. These, as lack of optical memory, are mainly due to the infancy of optical components and optical technology.

\subsubsection{Header Processing}

One of the really difficult problems is the switching speed needed. The network control and routing is performed electronically and the routing information from the packet header has to be converted to electrical form. In the KEOPS project this problem was solved by transferring the payload and the header with different transmission rates. The header was transmitted with relatively slow rate that made it possible to read the header to electronic form, while the data was transmitted fast in order to take the benefit from the transmission media. The data rate can be increased by compressing more data into the same time slot. In KEOPS project the header and the data were sent consecutively separated with a guard time. In optical burst networks and in photonic slot routing networks headers are sent in their own channels. In burst switching networks the header is transmitted before the burst and in photonic slot routing the header is transmitted simultaneously with payload. The main idea is the same in all these approaches: headers are sent slowly enough to read the control information and higher throughput is accomplished by compressing the payload and increasing the data rate. 


\subsubsection{Synchronization}

Most optical switches proposed so far are synchronous switches with constant length packets, i.e., the networks are slotted. This makes controlling and managing the switch easier and facilitates routing and buffering. On the other hand, careful synchronization is demanded. Synchronization is usually managed with optical delay lines. An example of this is the synchronizer implemented in the KEOPS project.

\subsubsection{Regeneration of Optical Signals}

In the KEOPS project optical 3R regenerator was implemented and tested. The problem is that using this kind of regenerator in real networks will be expensive. However, as the time goes on, the components will become cheaper and more mature. Optical regeneration is therefore not the hardest problem in this area.

\subsubsection{IP over Optical Networks}

IP protocol is today widely used. The bursty nature of the Internet traffic is one cause for the need for optical packet switching. Combining optical packet switching and IP protocol is not an easy task. A typical demonstrator for optical packet switching network is a slotted network with fixed packet size. Because IP packets are of variable size, they have to be either concatenated or fragmented [TZ99]. Because the data rate in optical packet switching networks is usually based on connecting considerable amounts of data to a header, concatenation seems to be a better approach. On the other hand, optical burst networks are usually asynchronous and use variable length packets, which makes them even more suitable for this purpose.

Another alternative is to use variable length packets. This kind of switches have been studied in [CCR02]. The throughput obtained with variable length packets in unslotted networks is better than in slotted networks. However, controlling the switch is more difficult, and with current relatively immature technology, these are difficult to implement. Optical burst switching seems to be an attractive alternative.

\subsection{Alternatives and Comparison}

\subsubsection{Optical versus Electronic Networks}

Optical fiber has several benefits in comparison with electronic cable. More data can be transferred with an optical fiber, which is thinner, cheaper and lighter than the corresponding copper cable. Additionally, the maximum data rate of an optical fiber is very high and the data rate of a specific network can be easily raised when the technology develops. There is no electromagnetic interference. 
The data can be transferred over longer distances without regeneration. Another advantage is the inherent security of optical fiber. Wiretapping is difficult in optical networks, and even if someone would succeed in it, this will be easily discovered [Red98].

The main weakness of optical networks is the infancy of technology. The mature technologies that work in electronic networks might be inefficient or totally useless in the optical environment. Yet, the first optical networks were developed decades ago, and they have developed remarkably. However, there are still only a few standards. The development of standards is slow, while the development of new technologies is fast. These are problems that every new invention confronts. The problems special to the fiber include the interference with gamma radiation and the high-voltage electrical fields [Red98]. Additionally, bending the fiber is a problem, because it changes the angle in which the signal meets the interface of the core and the cladding. If the bend radius is too tight, a fraction of the light might be lost.

It is generally believed that despite of these problems the fiber is a superior transmission medium and optical networks will replace electronic ones [Red98]. Several networks are still based on electronic transmission media because rebuilding networks costs much. Although the old networks are not as efficient as the new ones, it is too expensive to tear them down and build new ones. However, optical networks will become more and more common and finally totally supersede electronic networks.

\subsubsection{Optical Versus Electro-Optical Devices}

At the end of the 1990s many components used in optical networks were hybrid, i.e., they were partly optical and partly electronic. An example of this kind of component is a regenerator which first converts the signal to electronic, and then makes a new optical signal, i.e., the optical signal is read and according to that information, a new signal is created with a laser. Today most components can be all-optical, that is, no electro-optical or opto-electronic conversions are made. Electronics are slow compared to optics, and conversions take time. Therefore it is prefered to use all-optical components. An exception is header processing. Optical packet header is converted to electronic form and the switching process is controlled electronically.

\subsubsection{Packet Versus Circuit Switching in Optical Networks}

Today's all-optical networks are in practice circuit switched. Optical packet switched networks are on laboratory level, and it seems that in the optical world circuit switching is considerably more suitable alternative than packet switching. On the other hand, tomorrow's traffic will remain bursty. In any case, the traffic is in packet form on the higher levels. If a way to perform optical packet switching 
economically is found, it is clear that it would be a superior technology. However, as long as the components are immature and the technology is in its infancy, the circuit switching remains as an attractive alternative.

\subsubsection{Burst Switching Versus Traditional Packet Switching}

Optical burst switching is an intermediate form between circuit and packet switching. The data are sent in bursts, which can be as short as one packet or much longer. In burst switching a control packet is first sent to reserve resources for incoming bursts. From this on there are three alternatives. It is possible to first allocate the channels and wait for an acknowledgement packet from the destination node before the burst is sent (tell-and-wait). Today most proposals are one-way protocols. This means no acknowledgement packets are sent. There is still two alternatives: either the burst is sent immediately after the control packet (tell-and-go) or there is some kind of delay, offset time, before the burst is sent. In the first case, if there are resources, the burst is transmitted and otherwise it is rejected. The latter, and probably the most attractive alternative was to wait a short time and then send the burst. This alternative can be improved by reserving the resources from the moment the burst arrives to the node to the moment it will be served (just-enough-time).

Burst switching is a very promising switching alternative at the moment. It is believed, that it will become commercially available sooner than the packet switching. A commercially attractive burst switching network is easier to implement compared to optical packet network because it avoids two main problems: high switching speed and optical buffering. The bottleneck of optical packet switching network is header processing in the switches. Because data is concatenated into larger elements in burst switching networks, there are more data per header than in packet networks. Therefore, a higher data rate is obtained with the same header processing rate. Additionally, no complex buffering elements are necessarily needed. Bursts can be buffered in electronic form at the edge of the network instead of buffering at each node for the time the header is processed. Therefore switches can be implemented without buffering or with a few delay lines for contention resolution. Burst switching avoids the problems of packet switching, but is suitable for today's bursty traffic. In a few next years, it is clear that burst switching will be more attractive than optical packet switching. And in the long run burst switching seems to be the strongest competitor for optical packet switching.

\subsection{The Future of Optical Packet Networks}

Optical packet networks were first studied about ten years ago. Since then many things have changed, components have been improved, and on the other hand, the 
nature of the traffic has changed. Many problems are still unsolved, but optical technology starts to show signs of maturity. Optical networks spread from long haul networks to access area, and the networks are more complex, more efficient and more reliable than earlier [DL02]. Optical packet switching will probably remain at laboratory level for many years but optical burst switching has all opprtunities to become a practical technology possible already during next few years. And, even if optical packet networks will remain in their infancy in the near future, the improvement of optical circuit switching, optical technology and components will improve the future possibilities of optical packet switching networks. 


\section{Chapter 7}

\section{Simulations of the JET protocol in Burst Switching Network}

\subsection{JET Protocol}

The simulated system is an optical burst switching network, where JET (Just Enough Time) protocol is used for resource allocation. The JET protocol, first proposed by Xiao and Yoo in 1997 in [YX97a, YJQ97], has received a lot of attention in research community and has been studied, e.g., in [YX97b, GDSB01].

At the edge of the network packets are gathered to bursts of variable length. A control packet (i.e., burst's header) is then sent in a separate channel to allocate channels for the transmission. The burst waits at the edge node a certain time, and is then sent along the path the control packet has allocated for it. The time a burst waits at the edge node is called the offset time. The offset time is at least as long as the time it takes to process the control packet in the intermediate nodes. This means that there is no need to buffer the packet in the intermediate nodes. If there are enough free resources, the burst can be sent directly through the network without buffering, and it arrives to the destination node immediately after the control packet. Figure 7.1 illustrates the situation.

If control packet is unable to allocate a channel, it sends a NAK packet back to the source node. The NAK packet frees the allocated channels along the path. If the offset time is long enough compared to the propagation delay, the NAK packet might catch the burst before it is sent. If the burst has already been sent before the NAK packet arrives at the source node, the NAK packet meets it at some node along the path, and the burst is removed there. The NAK packet then continues to the sending node and informs it that the burst was lost.

Another advantageous feature of the JET protocol is the fact that the resources are reserved only for the time they actually are used. The control packet allocates a channel from the arrival time of the burst to the departure time of the burst. This additional information gives benefits over the traditional system, where the only information in the node is whether the channel is free or in use. 


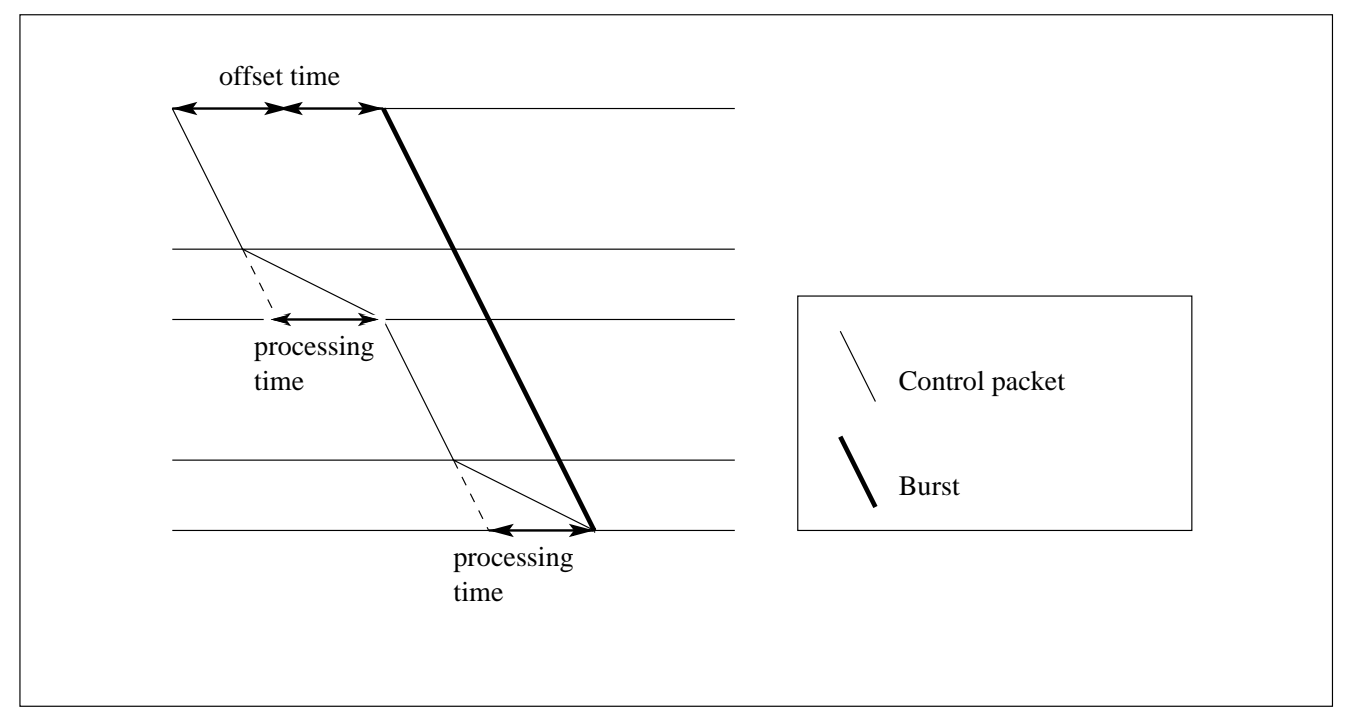

Figure 7.1: The JET protocol

A priority class based version of the JET protocol based on different offset times was proposed in [YQ98]. The idea behind this version is that longer offset time reduces loss probability because the control packet that tries to allocate a channel for the moment $t_{2}$ at the moment $t_{1}-\delta$ always wins a control packet trying to allocate the same channel for the same time slot at the moment $t_{1}$. A drawback in this scheme is the additional delay the higher priority classes experience.

Earlier research on the JET protocol (e.g., [YQ98, GDSB01]) assumes that the wavelength conversion can be performed in every node along the path. In the present work the JET protocol is studied in networks without wavelength conversion capability. The efficiency of the network suffers from this, but as will be shown, with reasonable number of optical buffers, i.e. delay lines, the loss in a network without wavelength conversion can be decreased almost to the same level as in a network with wavelength conversion. Optical delay lines are particularly suitable for networks using the JET protocol, because if the control packet is unable to allocate the channel for a certain time slot, it knows when the desired channel will be available again. Therefore, the time the packet has to be buffered is known beforehand, and a suitable delay line can be chosen and allocated according to that information. The control packet is updated and the channel is allocated starting from the moment the burst will be out from the buffer.

\subsection{Network Model}

Two different networks were studied. First, a small network with five core nodes was simulated. Then simulations were performed also for a larger network with 15 core nodes. In both networks, several edge nodes send bursts to each of the 
core nodes. Both networks were studied a) with wavelength conversion, b)without wavelength conversion and c) without wavelength conversion but with a number of optical buffers. In a network without wavelength conversion, a burst is lost, if the required wavelength is not free, whereas in the case with wavelength conversion the burst can be converted to any of the free wavelengths.

In both networks the following assumptions are made:

- Number of wavelength channels per link is 40.

- Each node sends traffic to each of the other nodes as Poisson stream with the same intensity $\lambda$.

- Processing time at nodes is assumed to be constant, $10 \mu \mathrm{s}$.

- Data rate is $10 \mathrm{Gbit} / \mathrm{s}$ at each channel.

- Burst lengths have uniform distribution between $5 \mu$ s and $15 \mu \mathrm{s}$. This corresponds to a burst size of 50 - 150 kbit.

- Buffer lengths are multiples of the maximum burst size.

- There is an optical buffer system for each output channel.

- All links are bidirectional.

In the small network with 5 nodes each link has the same propagation delay of $100 \mu$ s corresponding to approximately a distance of $2 \mathrm{~km}$. In the larger network links are of different lengths.

\subsection{Simulation Program}

The simulator was implemented using $\mathrm{C}++$ and the CNCL simulation library. The simulator takes care of the actual network implementation, packet generation and collection of statistical data. Additionally, Mathematica was used for generation of a large random network used in simulations. The number and location of nodes and links determined by Mathematica was written to a file. The $\mathrm{C}++$ simulator then read the file and generated a network corresponding to the output got from Mathematica.

As presented in Figure 7.2 the network consists of nodes, links and channel systems. Each node is connected to its neighbor nodes with links. Control packets and NAK packets are transferred from one node to another along the links. Each link has a corresponding channel system, which is a database of allocated channels within the links. The actual bursts are not transferred, but the statistics are gathered from failed and successful allocations. The node class has a child class called edge node. Each edge node is connected to one or more generators that generate control packets. Furthermore, each generator corresponds to one traffic class (traffic from node $i$ to node $j$ with specific parameters). A controller 
connects traffic to generators. If an allocations fails, the corresponding control packet is destroyed and a NAK packet is sent back along the path control packet arrived. The NAK packet deallocates the allocated channels on the path.

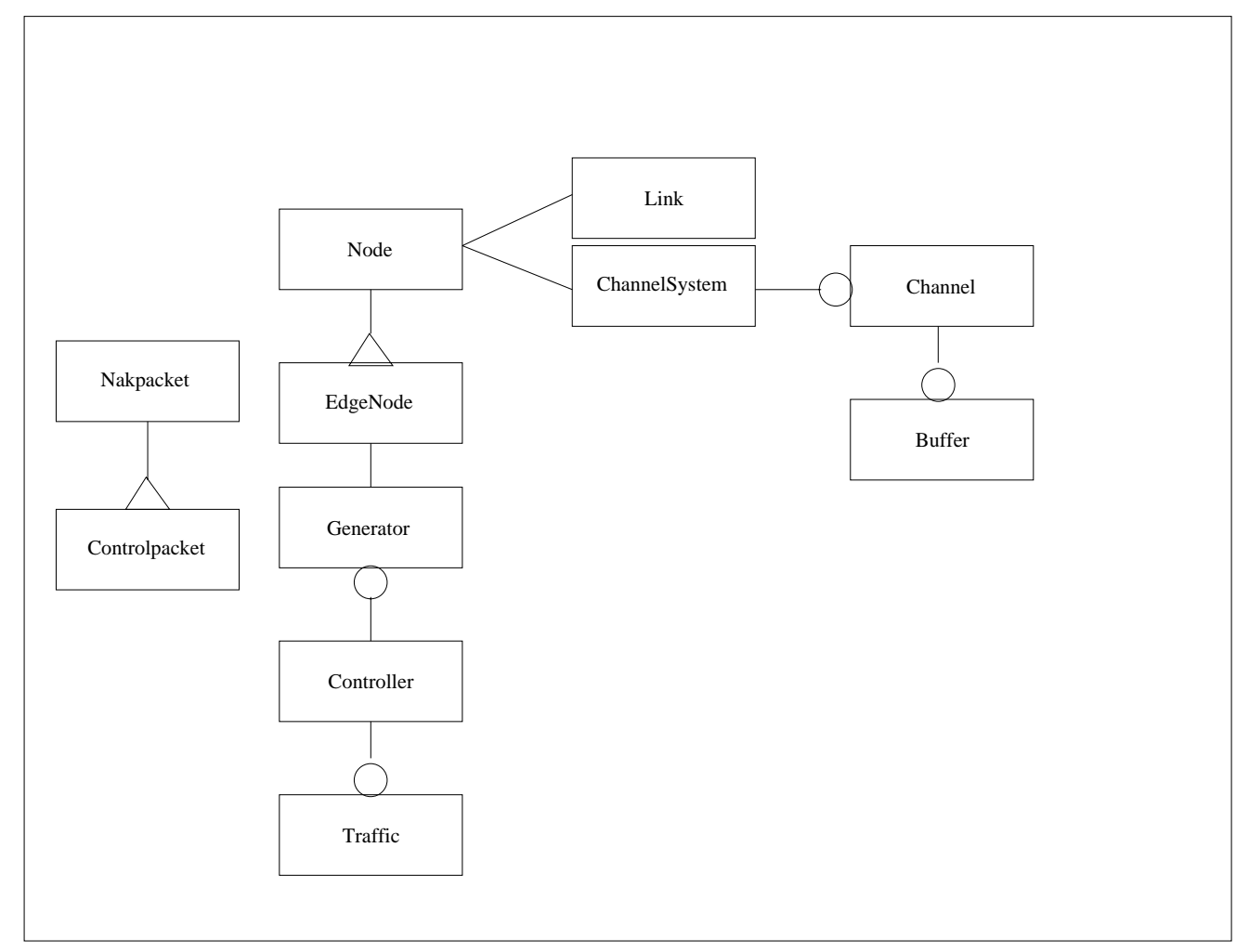

Figure 7.2: Structure of the simulator used

The simulation system is event based, which means that generators, links and nodes communicate by sending events to each other. They are all child classes of the EventHandler class implemented in CNCL library. An event handler is a state machine the function of which depends on the arriving event and optionally on the state. An event contains information of the sending and receiving event handlers, sending time and scheduling time, an identifier, a type and a priority value. The event scheduler controlling the program can send a certain event to a certain event handler at the right moment according to that information. The receiving event handler then chooses the routine to be executed according to the type of the event. The events sent in the simulator are illustrated in Figure 7.3.

Each event sent corresponds to a control packet or a NAK packet. First, a generator puts a generated control packet to an edge node's queue and sends EV_GET_G event to that edge node. The edge node takes the packet from the queue, processes it, puts it on a queue of a specific link and informs the link from the new packet with EV_GET_C event. The event is sent with a delay corresponding to the propagation delay on the link. Therefore, the link receives the event after the delay and the packet waits in the links queue a time corresponding to the propagation delay. Similarly, after receiving the event, the link puts the 


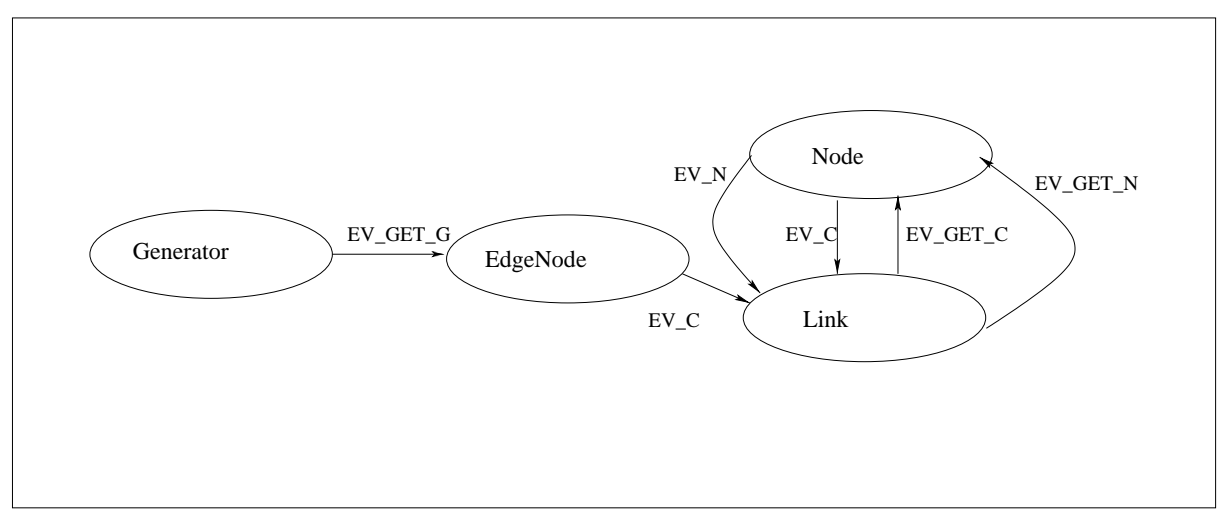

Figure 7.3: Events sent between the event handlers

packet into a queue of the next node and sends an event to that node with a delay corresponding to the processing time in the node.

If an allocation fails, the node sends a NAK packet towards the source node. The NAK packet frees all the allocations that are made for the corresponding burst. NAK packets are sent similarly as the control packets. Each node puts a NAK packet received to the queue corresponding to the next link and sends an event to the link, and each links forwards packets to the nodes. 


\subsection{Small Example Network}

First, a small example network with five nodes was studied. This example aims to show the main problems and questions that arose while studying the JET protocol without wavelength conversion. Loss probabilities of different connections are studied, and the effect of different offset times is discussed. The network illustrated in Figure 7.4 consists of five nodes and bidirectional channels connecting them. Each node sends traffic to each of the other nodes as a Poisson stream with the same intensity $\lambda$.

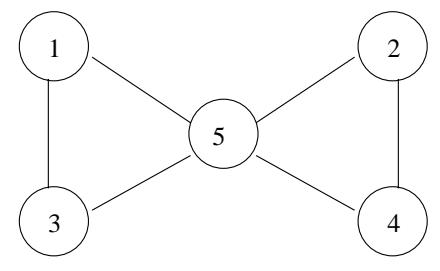

Figure 7.4: The little example network studied

Traffic in this network can be divided into four different traffic classes. Figure 7.5 illustrates the classes.

- Class 1 is constituted of traffic $1 \rightarrow 3,2 \rightarrow 4,3 \rightarrow 1$ and $4 \rightarrow 2$. This traffic does not compete with other classes, but has the whole link solely for itself.

- Class 2 contains traffic from the other nodes to node 5. This class contends for the resources with class 4 . For instance, the traffic $1 \rightarrow 5$ uses the same resources as the traffic $1 \rightarrow 2$.

- Class 3 contains the traffic from node 5 to other nodes. Also this traffic uses same resources with other classes. For instance, the traffic $5 \rightarrow 2$ competes with the traffic $1 \rightarrow 2$.

- Class 4 consists of the traffic $1 \rightarrow 2,1 \rightarrow 4,2 \rightarrow 1,2 \rightarrow 3,3 \rightarrow 2,3 \rightarrow 4$, $4 \rightarrow 1$ and $4 \rightarrow 3$. Unlike the other classes, this traffic has paths that are two links long.
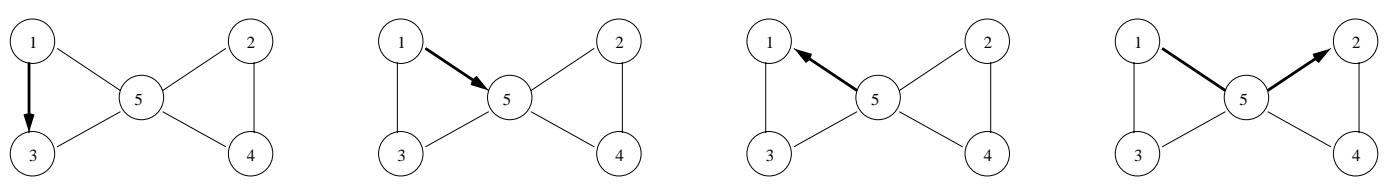

Figure 7.5: Traffic classes 1, 2, 3 and 4

Figure 7.6 demonstrates the performance of the systems with wavelength conversion and without it. The figure in the left hand side demonstrates the loss 

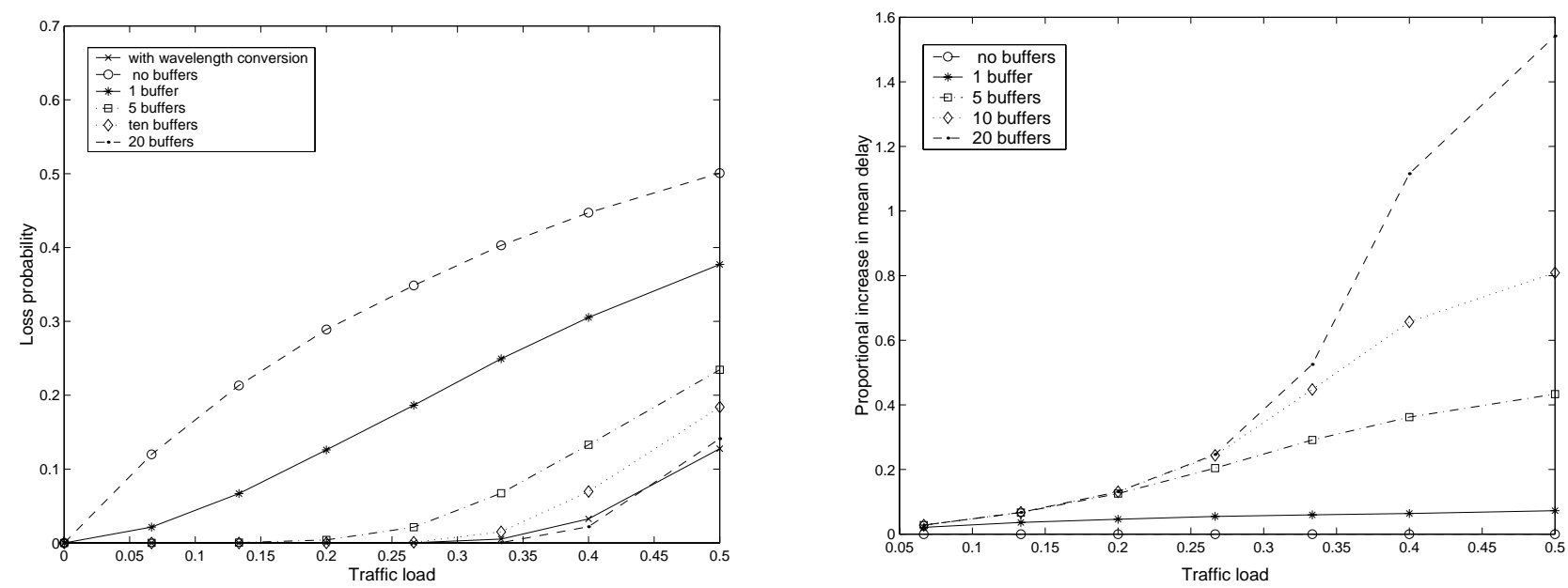

Figure 7.6: Loss probability and mean delay in the example network

probability, and the figure on the right hand side illustrates the proportional increase in the mean delay compared to the basic delay, i.e., the time packet is delayed, if it is not buffered. Average traffic load in the $\mathrm{x}$ axis is defined as

$$
\rho=\frac{\lambda_{\text {tot }} \frac{1}{\mu}}{W L}
$$

where $\lambda_{\text {tot }}$ is the total arrival intensity, i.e., 20 times the arrival intensity $\lambda$ of one connection, $W$ is the number of channels, $L$ is number of links and $\mu$ is the mean length of a burst. Note that the equations gives the average load and individual traffic loads at different links in this system vary a lot.

As can be seen from Figure 7.6, the blocking performance of the network with wavelength conversion is better than the performance in the network without wavelength conversion. However, with delay lines this difference in blocking probability can be remarkably decreased. A network without buffers and a network with one buffer per each channel suffer from loss even with small traffic loads. The network with 5 buffers has a low loss as long as average traffic load is beneath 0.2 . Later it will also be shown that although the average loss is smaller in the system with wavelength conversion, the buffered system has some other benefits. With ten buffers the loss can be further decreased, and by adding the number of delay lines even more, the same level as with the wavelength converted version could be achieved. However, adding more buffers is not reasonable, because if the delay lines are as long as the links, it would be better to add more links instead of buffers.

Using optical delay lines increases the average delay in the network. The figure on the right hand side illustrates the proportional increase in the delay compared to the delay in the system without buffers. The proportional increase in the delay is 


$$
d=\frac{D_{\text {measured }}-D_{\min }}{D_{\min }},
$$

where $D_{\text {measured }}$ is the actual delay and $D_{\text {min }}$ is the minimum delay possible, i.e., the delay if no buffers are used. The increase in the delay is relatively small when traffic loads are low, and only minor proportion of packets is used. When traffic load increases the delay increases. Especially, if the number of delay lines is high, the mean delay increases rapidly with average load between 0.3 and 0.4 . If the traffic load is higher, the mean delay does not increase as fast as with this specific load, because all the buffers are already in use, and only few new packets can be buffered, i.e., additional traffic is mostly lost. With a few delay lines extra delays remain low. In the 1-buffer system the increase is less than $10 \%$ and in the network with 5 buffers the increase is less than $10 \%$ with low load and from $20 \%$ to $40 \%$ with a higher load.

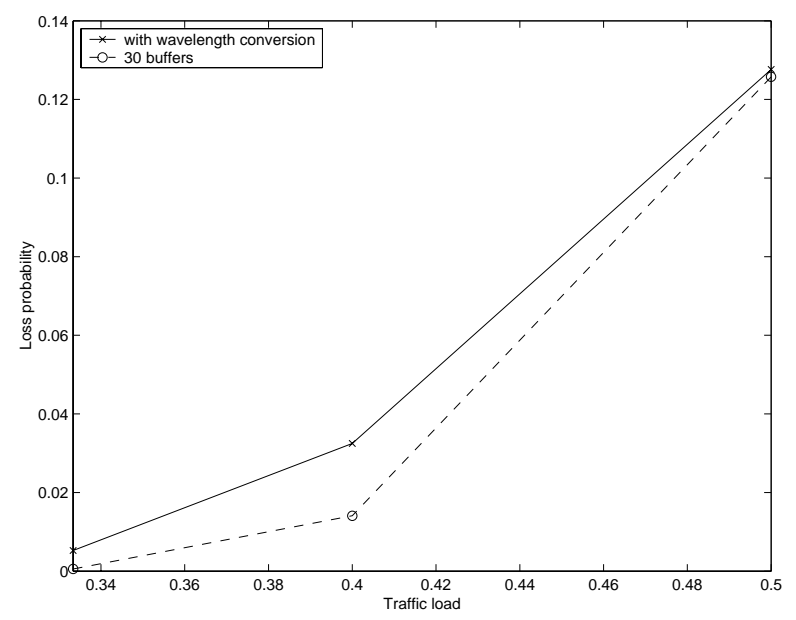

Figure 7.7: Loss probability in network 30 buffers

Figure 7.7 illustrates the loss probability in a network with 30 buffers per channel compared to the network, in which wavelength conversion is used. Each delay line in this system is a multiple of a third of the maximum burst length. With this system the loss smaller than in the system with wavelength conversion is obtained. 


\section{Performance of Different Connection Types}

Let us then study the behavior of different traffic classes in more detail. Figures 7.8, 7.9 and 7.10 illustrate the loss and proportional delay increase for different traffic class types in networks with different numbers of buffers. Traffic load in those figures is the average traffic load introduced earlier. The actual load each connection confronts depends on the number of connections per link and loss in the other channels. For instance, if average load is 0.5, traffic load on the link from node 1 to node 3 is actually 0.3 , while traffic load at link from node 1 to node 5 is much higher.

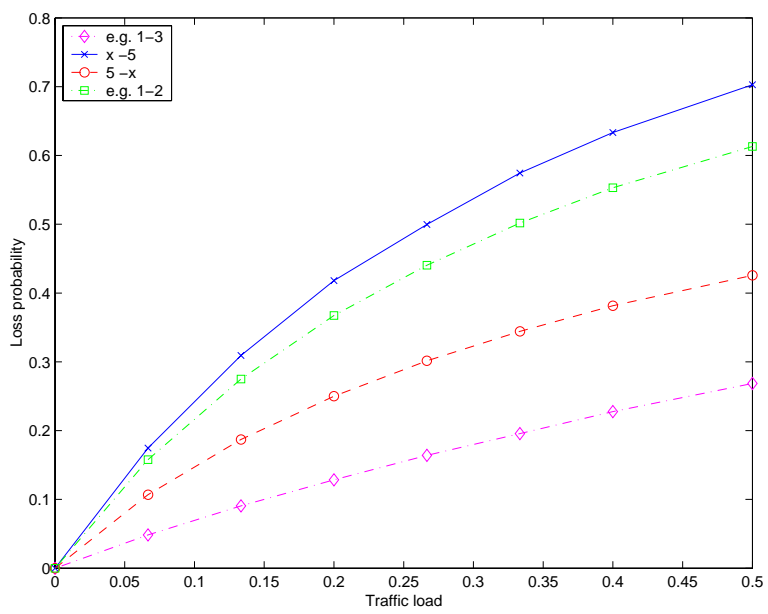

Figure 7.8: Loss of different traffic classes in network with no buffers

As can be seen from Figure 7.8, traffic class 2 from other nodes to node 5 experiences the greatest loss. Because the longer path means that also the offset time is longer, traffic class 4 (e.g., a traffic from node 1 to 2 ) always can allocate resources before traffic class 2 . On the other hand, traffic class 4 suffers from the loss in the second node, and many bursts are lost also in this traffic class. Traffic class 1 experiences minor loss compared to the others. This is natural, because this traffic does not compete with any other traffic.

When one buffer per channel is added to the network, loss decreases remarkably at every traffic class, as can be seen from Figure 7.9. Traffic class 1 obtains the greatest benefit. Otherwise the figure is quite similar to situation with no buffers. Class 2 from other nodes to node 5 experiences the greatest loss and class 4 with longest path confronts the second greatest loss. In a network with five buffers the relationship between different classes changes. All losses decrease, but the loss of classes 2 and 4 decreases more than the loss of traffic class 3 . When a burst that belongs to the traffic class 4 is buffered in the first node, the offset time between the control packet and the burst increases. Therefore class 4 is in a better situation than class 3 in node 5 . The loss of class 4 decreases, and it can allocate resources that otherwise would be used by the class 3 traffic. Therefore, the loss of class 3 becomes greater than the loss of the other classes. This did not happen when there was only one buffer, because in the first node on the path of 

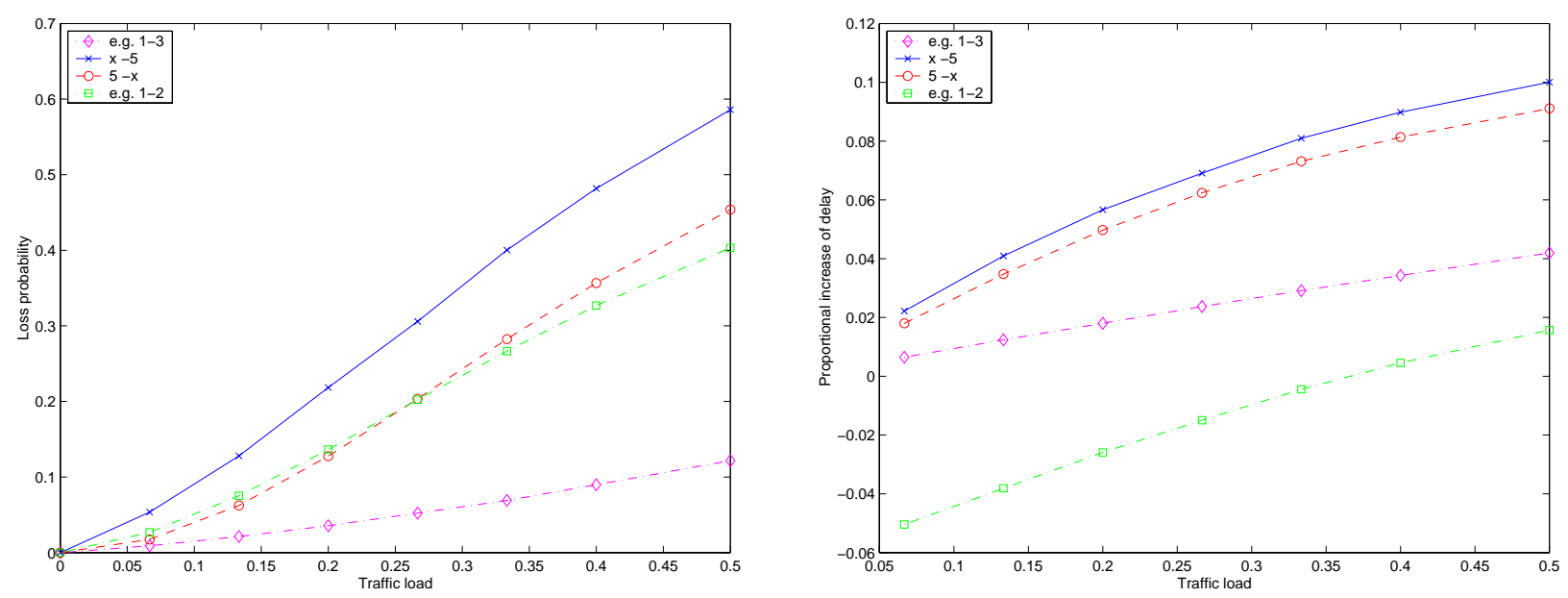

Figure 7.9: Loss and delay of different traffic classes in network with one buffer per channel

class 4 , class 2 confronts more loss, and buffers were mostly used by it. Situation in networks with more buffers per channel is similar to the network with 5 nodes per channel.
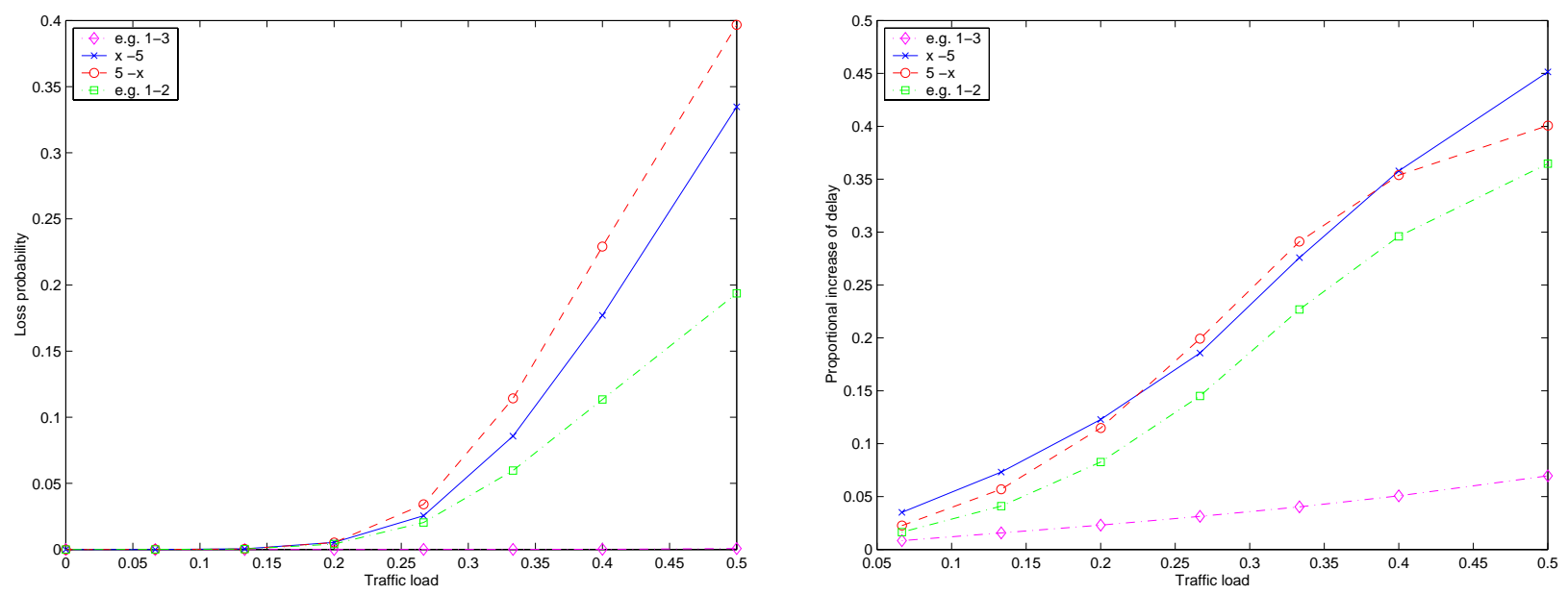

Figure 7.10: Loss and delay of different traffic classes in network with 5 buffers

Figure 7.11 illustrates the loss probability in a network where wavelength conversion can be performed. Although the total loss is small, smaller than in the network with 10 buffers per channel, the loss probability is very unfairly distributed among different connection types. Connections from other nodes to node 5 suffer from a great loss, while the loss of other connection types is nearly zero.

Figures 7.9 and 7.10 illustrate the mean delay of different traffic class types in networks considered above. When there are no buffers, the delay is the same for all the bursts, except that traffic class 4 has twice the delay the other classes have. Total delays are around $100 \mu \mathrm{s}$ and $200 \mu \mathrm{s}$. The proportional increase in the delay of each class illustrated in the figures is given by Equation 7.2. 


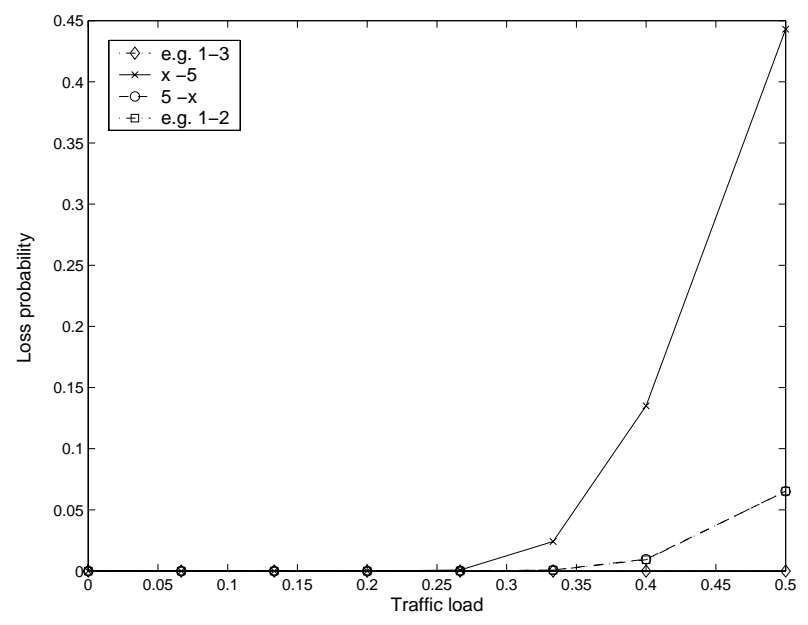

Figure 7.11: Loss of different traffic classes in network with wavelength conversion

So far the mean loss and delay of different traffic classes have been studied. Figures 7.12 and 7.13 illustrate the loss each class suffers on different links. In the network without buffers, traffic class 2 , at links from other nodes to node 5 , has the highest loss. Class 3 in the link from node 5 to other nodes has the second highest loss. Class 4 has higher loss on the second link, i.e., on links from node 5 to the destination nodes. Class 4 gets additional benefit from its offset time on the second link. This causes the great loss of class 2. However, the loss on the second link is lower. The actual load class 4 experiences on the second link is smaller, because the loss in the first link is as high as 0.3. Additionally the traffic is smoother than in the first link, because the peak traffic is already dropped. Traffic class 1 is not illustrated in the figure, because it is not dependent of the other traffic classes and its behavior can be seen from the figures considering traffic classes.
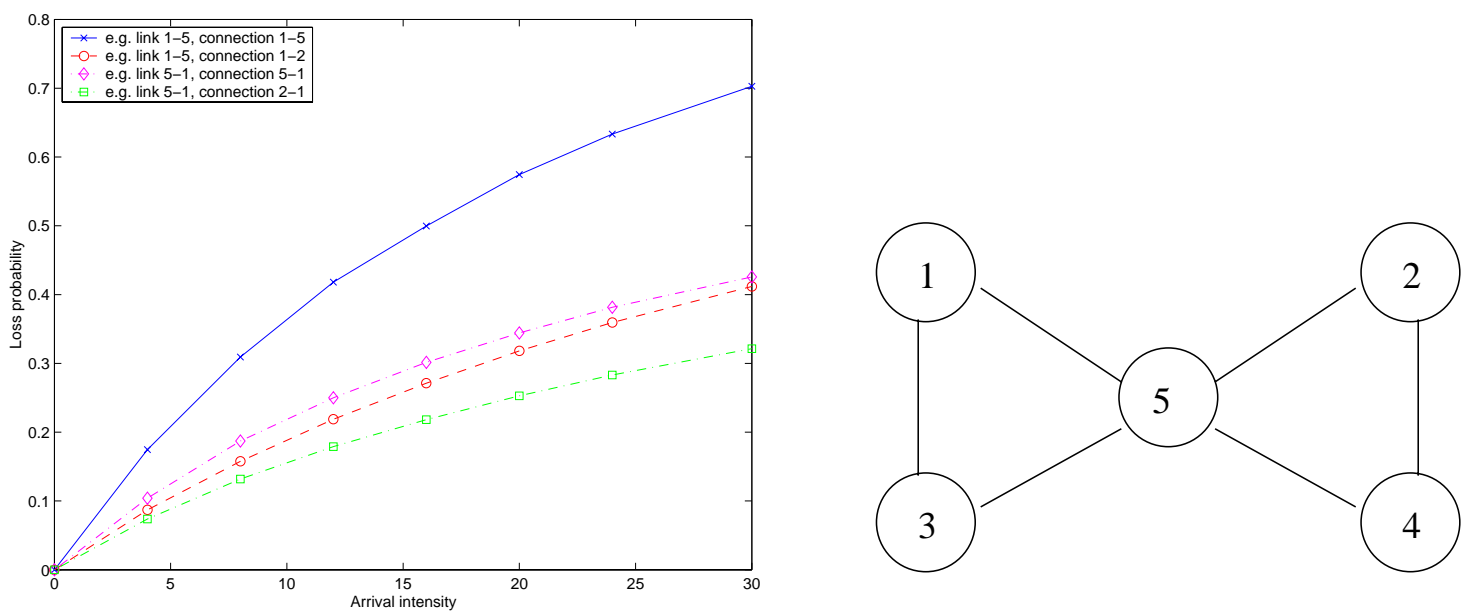

Figure 7.12: Loss of different traffic classes at different links, no buffers

The performance of a network with one buffer for every channel, illustrated in 
the left hand side of Figure 7.13, is quite similar to the network without buffers. The loss decreases, but the order of loss probabilities remains the same. The loss probabilities of traffic class 4 at different links approach each other. The performance of the network with 5 buffers per channel is illustrated on the right hand side of the figure. The loss probability of traffic class 4 at the links from node 5 to other nodes is low, and the loss probability of traffic class 3 at the same links is high compared to the other classes. As earlier explained, this is caused by the additional offset time that bursts in traffic class 4 get, when they are buffered while the control packets are forwarded. Also packets of the other traffic classes can be buffered, but because traffic class 4 has a two links long path, the probability of a packet to be buffered is greater for this class.
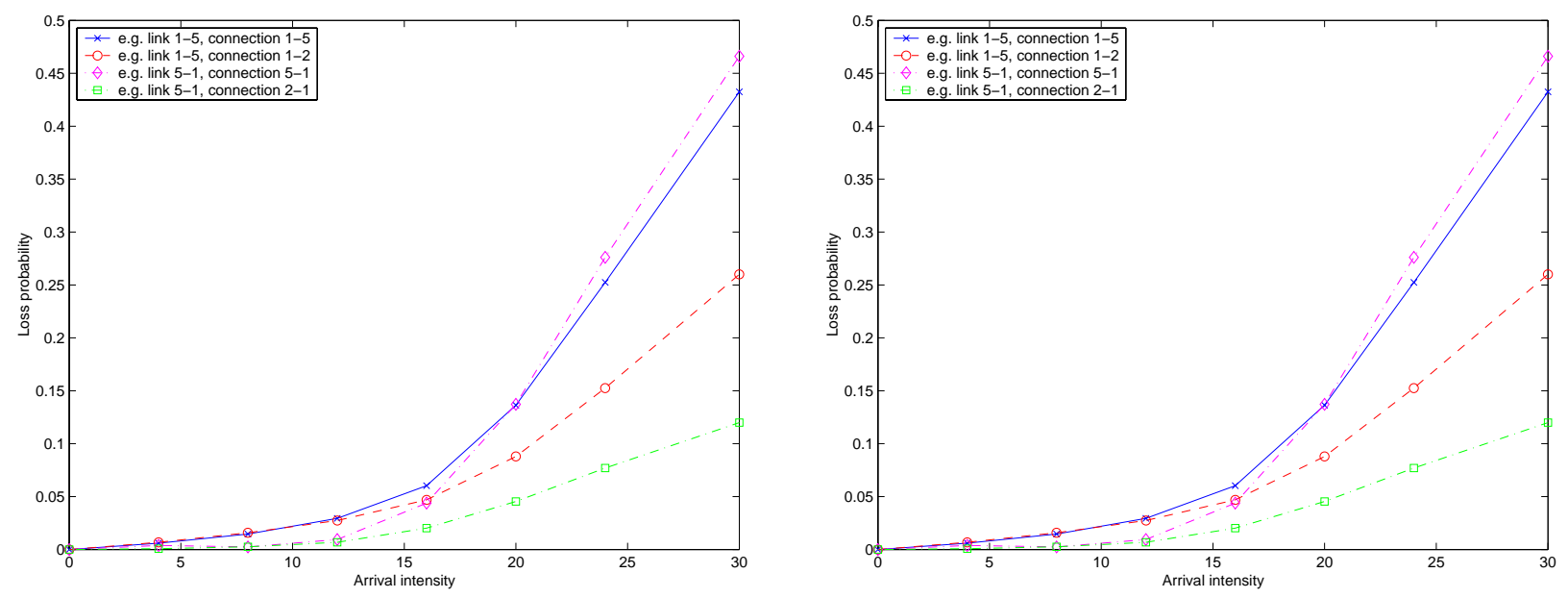

Figure 7.13: Loss of different traffic classes at different links, 1 buffer per channel and 5 buffers per channel 


\subsubsection{Different Delay Line Configurations}

In the examples above, the fiber delay lines have lengths that are multiples of the smallest length, i.e., configurations are type 1, 2, 3, 4, 5, where the shortest delay line has length corresponding to maximum packet length and longest corresponding to 5 packet lengths. This is not the most efficient configuration. Different fiber line configurations have been studied in [Sus00]. The results obtained were that random configurations with small and long lengths produce best results. This depends of course on the arrival process to the node. Three promising configurations, listed in Table 7.1, were studied and compared to regular configurations also listed in Table 7.1.

\begin{tabular}{|c|c|}
\hline \hline Configuration & Delay \\
\hline \hline 1 & $1,2,3,4,5$ \\
\hline 2 & $2,4,6,8,10$ \\
\hline 3 & $1,2,3,4,5,6,7,8,9,10$ \\
\hline 4 & $1,2,7,9,10$ \\
\hline 5 & $1,3,8,9,10$ \\
\hline 6 & $1,4,8,9,10$ \\
\hline \hline
\end{tabular}

Table 7.1: Different delay line configurations

In systems with fixed length packets configurations 1, 2 and 3 are FIFO queues, while configurations 4,5 and 6 are not. In JET the situation is not that clear, because the system contains both control packets and bursts. Control packets are not buffered and are in the same order through out the path. The order of the burst, however, can change. Figure 7.14 illustrates the situations with variable burst lengths and fixed burst lengths.
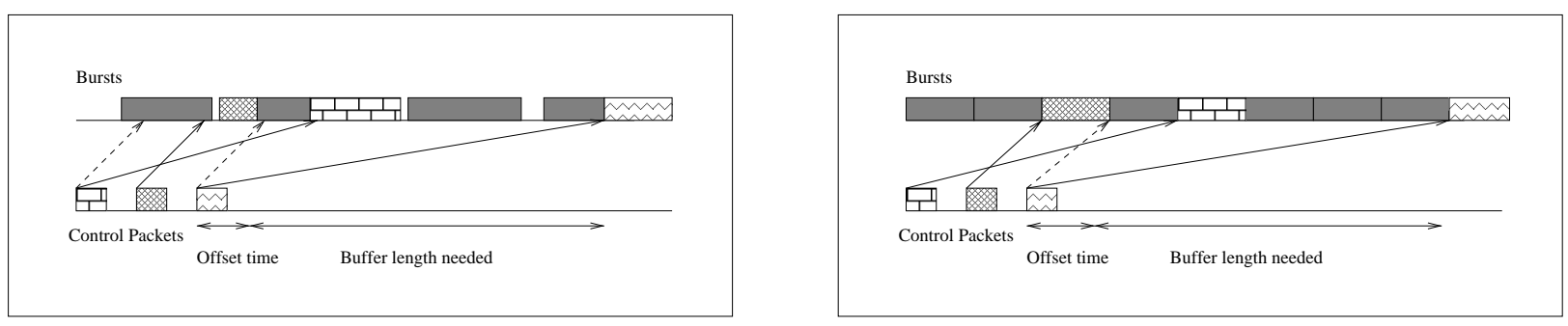

Figure 7.14: Buffers in systems with a) variable length bursts and b) fixed length bursts

With variable length packets, as in the simulations done in this work, it is possible that a burst is short enough to fit in a small gap that has been too small to the previous packets. Therefore, the first arrived but longer burst is directed to a longer buffer, while the shorter burst is switched directly or put into a shorter queue. Figure 7.14 illustrates this. The offset times are same for all the packets. 
The first packet is unable to allocate the time slot it wants, because there already is a burst. The first free time slot that is large enough is in the middle of the figure. The burst corresponding to the next control packet is shorter and can be put to the channel without buffering. The order of the bursts chances. None of the configurations holds the FIFO discipline with variable length packets, and using configurations 4,5 or 6 does not have any drawbacks compared to the first three configurations.

If the burst were of fixed length, the situation would be as on the right hand side of the figure. In the first three configurations control packets with similar offset times would allocate resources in order, but the control packets with different offset times would not. In the figure the first control packet has longer offset time than the other packets. It arrives to the node before the other control packets, but it wants to allocate a time slot after the allocation times of the other bursts. The first control packet tries to allocate the channel from moment $t_{i}$ to $t_{i+1}$ and successes in that. The second control packet tries then allocate the channel from moment $t_{i-2}$ to $t_{i-1}$. There is a free gap and allocation successes. The last packet tries to allocate the channel for the time slot from $t_{i-1}$ to $t_{i}$. The slot is already in use, and the next free slot is from $t_{i+4}$ to $t_{i+5}$, i.e., the burst corresponding to the first control packet will arrive to the node last but it will be sent before the third burst. With configurations 4, 5 and 6 also the order of burst with the same offset time might change.

Figures 7.15 and 7.16 illustrate the mean loss and delay of different configurations. Naturally, the configuration with 10 delay lines obtains best results. The irregular configurations get best results of the five delay line configurations, but the difference from the regular configurations is minor.

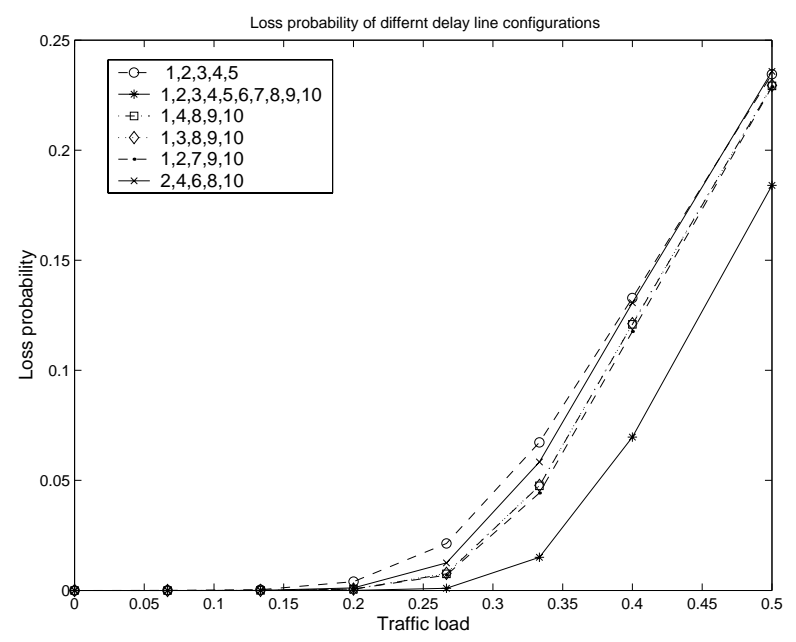

Figure 7.15: Loss of different configurations

Figure 7.17 illustrates the loss probabilities and proportional delay increases of the system with delay line configuration 6 . The figure is similar to the results obtained from configuration 1 . Also the configurations 4 and 5 are similar to con- 


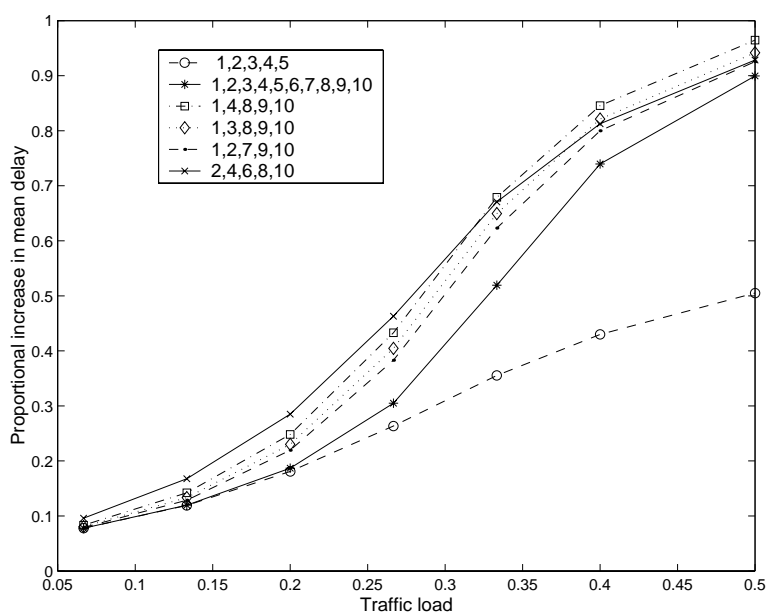

Figure 7.16: Delay of different configurations
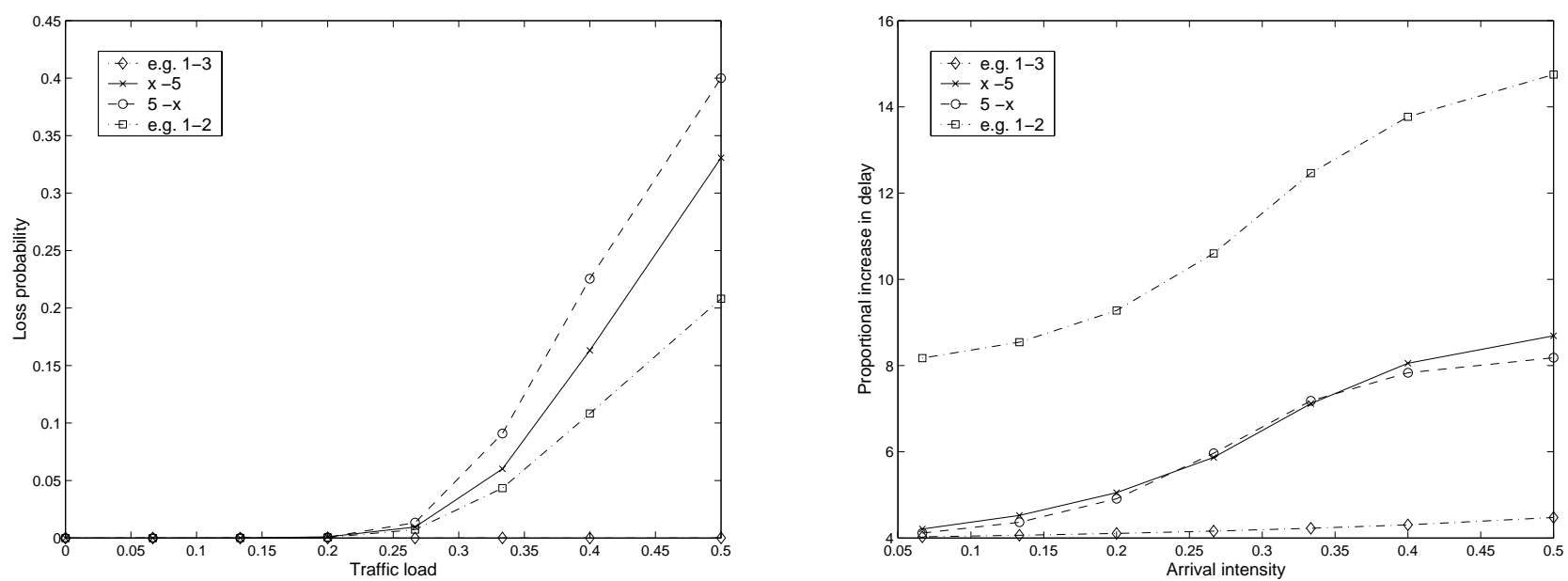

Figure 7.17: Loss and delay of the network with delay line configuration number 6

figuration 1 . The loss probabilities and delays are slightly smaller but there are no big differences and the relationship between different delay line configurations remains the same.

The benefit obtained with different configurations was relatively low. Possibly the performance could be increased by randomizing the delay line lengths more. In these simulations the delay line length was always a multiple of the maximum packet length. These delay lines could be replaced with delay lines that are e.g., one and a half times the maximum burst length. 


\subsection{Efficiency in Larger Network}

A random network with 15 core nodes, illustrated in Figure 7.18, was simulated in order to study the behavior of different schemes in a more realistic environment. The simulation parameters were mostly the same as in the small network. Each core node is connected to number of sources that send burst as a Poisson stream. The differences between the two networks are the number of nodes, and variablity of link lengths in the latter network. Simulations were again performed for network models a) with wavelength conversion, b) without wavelength conversion and c) without wavelength conversion but with 1,2 and 5 buffers per each channels.

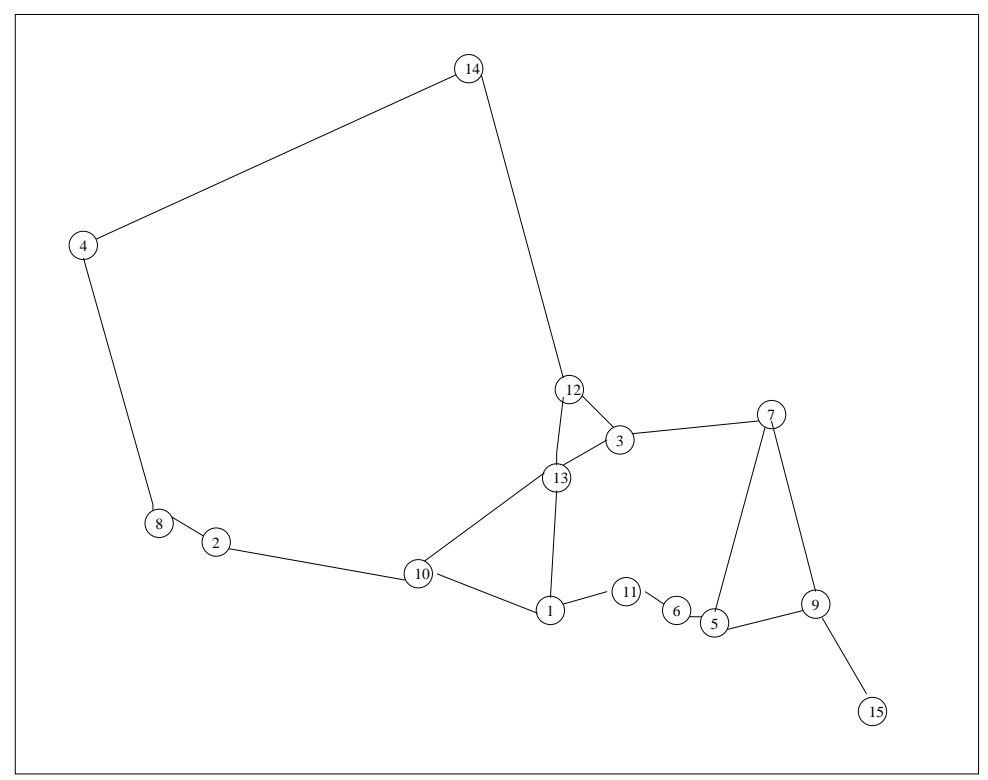

Figure 7.18: A random network with 15 nodes

The random network is generated with the help of Mathematica. The 15 nodes were randomly placed on a disk. The Gabriel algorithm was then used for determining which nodes were connected with a link and, finally, the shortest paths between the node pairs were determined with the Dijkstra algorithm. Figure 7.19 illustrates the function of the Gabriel algorithm. Briefly explained, two nodes are connected with a link, if there are no other nodes within the circle drawn through them. In the figure, nodes 1 and 2 are connected because the circle drawn is empty. Nodes 3 and 4 are not connected with a link, because node 2 is inside the circle drawn through the nodes. In practice networks generated this way look very natural.

The mean loss probabilities of systems with a different number of buffers are illustrated in Figure 7.20. If the load is small, the performance of the network can be remarkably improved by adding delay lines. With a larger average load, however, the benefit obtained from buffers is smaller. The proportional increases in delays of systems with 1, 2, 5 or 10 buffers per channel are illustrated in Fig- 


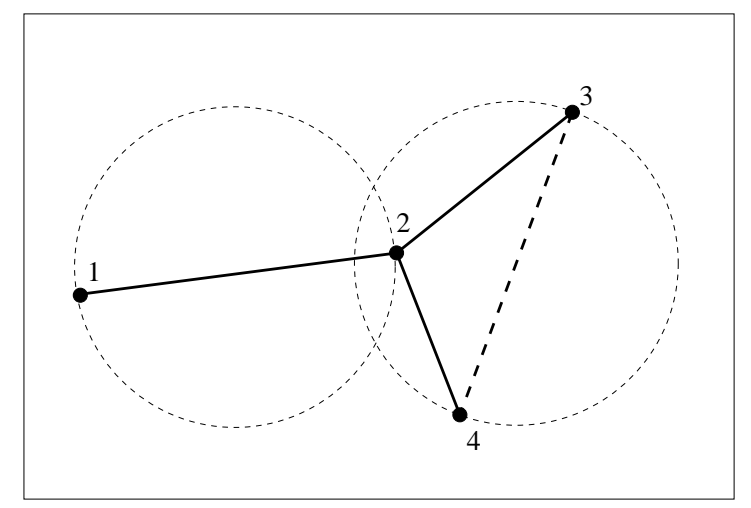

Figure 7.19: The Gabriel algorithm

ure 7.21. The increase in the delay remains relatively low.

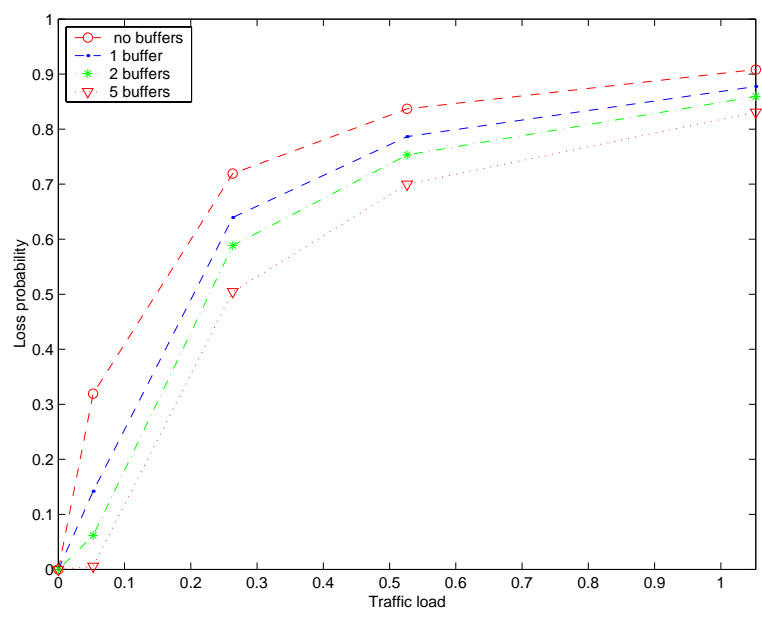

Figure 7.20: Loss probabilities in systems with 15 nodes

Figure 7.22 illustrates the delay in the system with wavelength conversion. As can be seen, in this system the loss is very low with small traffic loads but with larger load the loss increases. If the traffic load increases enough, the loss increases to the same level as in the buffered systems. In systems using JET protocol, longer connections have longer offset times on the first links. The offset time then decreases and is near to zero on the last links. Therefore connections have greater loss on the last links. With a low load a network with wavelength conversion performs better, because the long connections reach their destinations. However, when traffic load increases the long connections suffer from loss on the last links of the connections. In the networks with buffers the loss is more fairly distributed. Therefore, the wasted data is transferred longer and resources are lost in the network with wavelength conversion. 


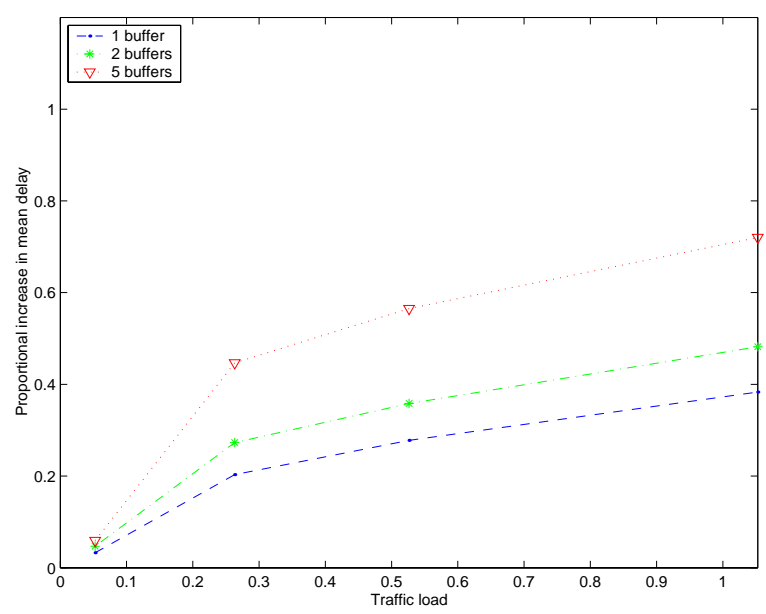

Figure 7.21: Proportional increase in mean delay in a system with 15 nodes

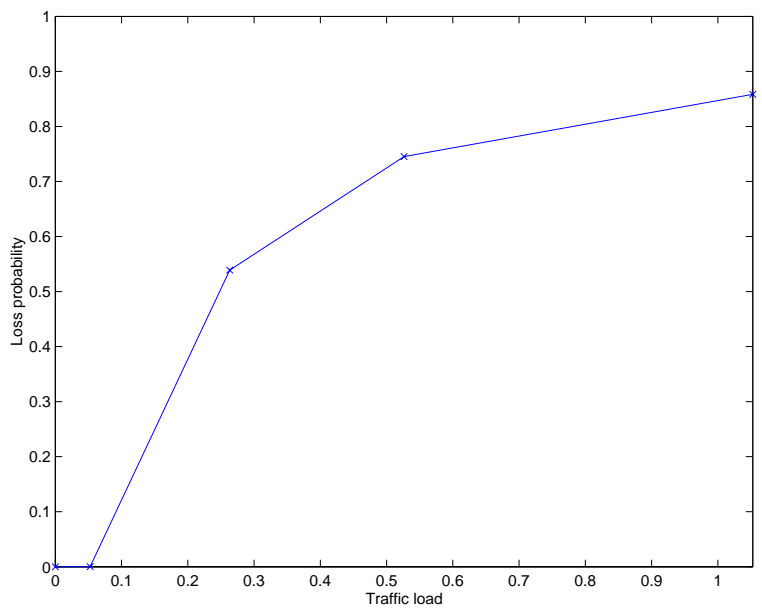

Figure 7.22: Loss probabilities in systems with 15 nodes, with wavelength conversion

\subsection{Discussion}

Two network models have been simulated with a number of different fiber line configurations. As can be expected, networks where wavelength conversion was not used suffer from a greater loss probability compared to systems with wavelength conversion. One is, however, able to decrease the loss probability with the aid of delay line buffers. A network with 40 channels per link and 20 delay line buffers per each channel produced roughly the same loss probability as one with wavelength conversion. In a small network with average load 0.5 , the delay increases to 2.5 times the delay obtained in a network without buffers. If the average traffic load is smaller than 0.35 the delay increase is less than half of the delay in a network with buffers. With this load or smaller, the performance of systems with 10 and 20 buffers are quite similar. If the traffic load is higher, same connections suffer from load that is greater than 1 . In small networks with relatively low load, a small number of buffers is sufficient to decrease the delay remarkably. 
An interesting observation relates to the behavior of different connection types. Although the system with wavelength conversion seems to have better average performance than the system with 10 delay lines, one connection class suffers from noticeably greater loss than the others. If the aim is to guarantee that each connection gets at least some minimum performance, even the system with 5 buffers achieves the same level as the system with wavelength conversion. The fairness of the system could be also decreased by adding an extra offset time to some of the connections. An approach similar to this, using extra offset times in order to divided the connections into different priority classes, is studied i.e. in [YQ98]. 


\section{Chapter 8}

\section{Conclusions}

In this thesis optical packet switching and optical burst switching were studied. A few recent research projects were reviewed in order to understand the evolution of optical packet switching, and the main problems in the area were identified. The first projects were started in the early 1990's. At first the focus was on ATM, and later transmitting IP packets over light and the idea of optical Internet gathered more interest. In the latter half of the 1990's the KEOPS project took place. Several new components and two prototypes of all-optical switches were implemented. After the KEOPS project the evolution of optical networks has been faster, and several other projects have been carried out. Implementation of WDM capable optical packet switches at the end of the 1990's was the next step on the road of the evolution of optical packet switching. During the few last years optical burst switching and other intermediate forms of optical packet and optical circuit switching have gathered more interest.

As already said, there have been several implementations of optical packet switches in laboratory environment, and several projects have been accomplished. The question is, when optical packet switched networks will be taken into commercial use. This will not happen until the optical packet switching obtains, with a reasonable price, better performance than other alternatives. As long as adding more cable is cheaper than implementing a packet switch, optical packet switching will not be a feasible commercial solution. It will take years before the optical technology has developed enough to support packet switching. The main reasons for this are the switching speed needed and the lack of optical random access memory. A few solutions that try to overcome the problems, and still offer the benefits of optical packet switching, have been proposed.

Photonic slot routing is a packet switching technology where fixed length packets are synchronized and the system is slotted in time domain. Each time slot contains several packets at different wavelengths. One channel is allocated for headers and the time slot in that channel contains the headers for all the packets in this specific time slot. This kind of system has of course poorer performance than a normal packet network, but it is easier to manage.

Another, and even more interesting, alternative is optical burst switching. As 
in photonic slot routing, the headers are transmitted in their own channel. The basic idea of optical burst switching is that the packets are gathered into larger packets, i.e., bursts, before sending. The header of a burst is called a control packet, and it is sent beforehand to allocate transmission channels for the burst.

In this work one of the protocols proposed for optical burst switching networks was studied. The JET protocol is complex but on the other hand its performance compared to other protocols used in optical burst switching networks is good. In this work the performance of JET protocol in networks without wavelength conversion was studied and compared to the performance of the wavelength converted version.

If wavelength conversion cannot be performed in each node of the network, the performance of the system suffers remarkably. On the other hand, the number of tunable wavelength converters needed in the network, where wavelength conversion can be performed, is large, and the price can be very high. If optical delay lines are used instead of wavelength converters, more cable and switching logic is needed. With the simulations it was shown that with relatively small number of buffers a performance that is comparable to that of a wavelength converted version can be achieved. 


\section{Bibliography}

[Ben97] J. Benoit. ATMOS ATM Optical Switching, Project Summary. Technical Report R2039, RACE, January 1997.

[CC97] M. Calisti and F. Callegati. Traffic Models for an Optical Transparent Packet Network. European Conference on Networks and Optical Communications (NOC'97), June 1997.

[CCR02] F. Callegati, G. Corazza, and C. Raffaelli. Exploitation of DWDM for Optical Packet Switching With Quality of Service Guaratees. IEEE Journal on Selected Areas in Communiacations, 20(1):190-201, January 2002 .

$\left[\mathrm{CFK}^{+} 96\right]$ I. Chlamtac, A. Fumagalli, L. C. Kazovski, P. Melman, W. H. Nelson, P.-L. Poggiolini, M. Cerisola, A. N. M. Masum Choudhury, T. K. Fong, R. T. Hofmeister, C-L. Lu, A. Mekkittikul, D. J. M. Sabido IX, C-J. Suh and E. W. M. Wong. CORD: Contention Resolution of Delay Lines. IEEE Journal on Selected Areas on Communications, 14(5):1014-1030, June 1996.

$\left[\mathrm{CHA}^{+} 01\right]$ M. C. Chia, D. K. Hunter, I. Andonovic, P. Ball, I. Wright, S. P. Ferguson, K. M. Guild and M. J. O'Mahony. Packet Loss and Delay Performance of Feedback and Feed-Forward ArrayedWaveguide Gratings-Based Optical Packet Switches With WDM Inputs-Outputs. Journal of Lightwave Technology, 19(9):1241-1254, September 2001.

[CJR $\left.{ }^{+} 98\right]$ D. Chiaroni, C. Janz, M. Renaud, P. Gravey, C. Guillemot, P. Gambini, P. B. Hansen, M. Schilling, G. Corazza, H. Melchior and I. Andonovic. KEOPS Final Report. ACTS Project KEys to Optical Switching AC043, September 1998.

$\left[\mathrm{CLJ}^{+} 98\right]$ D. Chiaroni, B. Lavigne, A. Jourdan, M. Sotom, L. Hamon, C. Chauzat, J-C. Jacquinot, A. Baraso, T. Zami, F. Dorgeuille, C. Janz, J-Y. Emery, E. Grard, and M. Renaud. Physical and Logical Validations of a Network Based on All-Optical Packet Swithcing systems. Journal of Ligthwave Technology, 16(12):2255-2264, December 1998.

[DKGS01] D. Dey, A. M. J. Koonen, D. Geuzebroek and M. R. Salvador. FLAMINGO: A Packet-switched IP over WDM Metro Optical Net- 
work. In Procs. of 6th European Conference on Networks $\&$ Optical Communications (NOC 2001), June 2001.

[DKS00] D. Dey, A. M. J. Koonen and M. R. Salvador. Network Architecture of a Packet-Switched WDM Lan/Man. In Procs. of the 2000 IEEE/LEOS Symposium - Benelux Chapter, October 30, 2000, Delft, The Netherlands., October 2000.

[DL02] S.S. Dixit and P.J. Lin. Optical Networking: Signs of Maturity. IEEE Communications Magazine, 40(2):64-64, February 2002.

$\left[\mathrm{DvBK}^{+} 01\right]$ D. Dey, A. van Bochove, A. M. J. Koonen, D. Geuzebroek and M. R. Salvador. FLAMINGO: A Packet-switched IP-over-WDM Alloptical MAN. In Procs. of 27th European Conference on Optical Communications (ECOC 2001), Amsterdam, NL, 2pp., September 30-October 4, 2001, October 2001.

[EL00] V. Eramo and M. Listanti. Packet Loss in a Bufferless Optical WDM Switch Employing Shared Tunable Wavelength Converters. Journal of Ligthwave Technology, 18(12):1818-1833, December 2000.

[EM00] Jaafar M. Elmirghani and Hussein T. Mouftah. Techologies and Architectures for Scalable Dynamic Dense WDM Networks. IEEE Communications Magazine, 38(2):58-66, February 2000.

[FMH97] B. Fabianek, K. Myken and A. Houghton. Optical Network Research and Development in European Community programs: From RACE to ACTS. IEEE Communications Magazine, 35(4):50-56, April 1997.

[GDSB01] C. Gauger, K. Dolzer, J. Späth and S. Bodamer. Evaluation of Reservation Mechanisms in Optical Burst Switching. AEÜ Int. J. of Electron. and Commun., 55(1), 2001.

$\left[\mathrm{HNC}^{+} 99\right]$ D. K. Hunter, M H. Nizam, M. C. Chia, I. Andonovicand K. M. Guild, A. Tzanakaki, M. J. O’Mahony, J. D. Bainbridge, M. F. C. Stephens, R. V. Penty and I. H. White. WASPNET A Wavelength Switched Packet Network. IEEE Communications Magazine, 37(3):120-129, March 1999.

[Int01] Raman Amplification Design in Wavelength Division Multiplexing (WDM) Systems. http://www.iec.org/online/tutorials/raman/ index.html, 2001.

[KBS01] A. Kushwaha, Sanjay K. Bose and Y. N. Singh. Analytical Modeling for Performance Studies of an FLBM-Based All-Optical Packet Switch. IEEE Communication Letters, 5(4):227-229, April 2001.

[KOS98] K. Kamakura, T. Ohtsuki and I. Sasase. An ATM-Based Optical Code Division Multiplexing Transport Network Using Coherent Ultrashort Pulses. 1998 IEEE Global Telecommunications Conference (GLOBECOM'98), November 1998. 
[KT01] I. Khrushchev and S. Turitsyn. Lecture material in Optical communication systems. http://www.eeap.aston.ac.uk/ ik/OptComm/Lectures/, 2001.

$\left[\mathrm{LDA}^{+} 99\right]$ P. Lagasse, P. Demeester, A. Ackaert, W. van Parys, B. van Caenegem, M. O’Mahony, A. Tsanakaki, K. Stubkjaer and J. Benoit. Roadmap Towards the Optical Communications Age. http://www.intec.rug.ac.be/Research/Projects/ horizon/roadmap_nov99.pdf, November 1999. Draft edition.

[LPTS01] C. Litvanyi, T. Punyasakhon, R. Tiyachate and N. Suwanpatra. A Technology Evaluation for Implementing NextGeneration All-Optical Switched Networks. The Interdisciplinary Telecommunications Program, Spring 2001 student capstone project presentations, April 2001. http://198.11.21.25/ capstoneTest/Students/Papers/docs/proceedingpaper39206.pdf.

[Muk97] B. Mukherjee. Optical Communication Networks. McGraw-Hill series on computer communications. McGraw-Hill, 1997.

[OSHT01] M. J. O’Mahony, D. Simeonidou, D. K. Hunter and A. Tzanakaki. The Application of Optical Packet Switching in Future Communacation Networks. IEEE Communications Magazine, 39(3):128-134, March 2001.

[Red98] IBM Redbooks. Understanding Optical Communications. http://master.math.unibuc.ro/documentatie/optic/Understanding _Optical_Communications0738400580/ewtoc.html, November 1998. ISBN: 0738400580.

[RMGB97] M. Renaud, F. Massetti, C. Guillemot and B. Bostica. Network and System Concepts for Optical Packet Switching. IEEE Communications Magazine, 35(4):96-102, April 1997.

[RS98] R. Ramaswami and K. N. Sivarajan. Optical Networks, A Practical Perspective. Morgan Kaufmann Series in Networking. Morgan Kaufmann Publishers, 1998.

[SdGD01] M. R. Salvador, S. H. de Groot and D. Dey. An All-Optical WDM Packet-Switched Network Architecture with Support for Group Communication. In Procs. of IEEE International Conference on Networking (ICN), Colmar, France, July 2001, July 2001.

[ST92] Y. Shimazu and M. Tsukada. Ultrafast Photonic ATM Switch with Optical Output Buffers. Journal of Ligthwave Technology, 2(10):265272, February 1992.

[Sus00] R. Susitaival. Optisen pakettimultiplekserin suorituskykyanalyysi. Helsinki University of Technology, Laboratory of Telecommununications Technology, Internal report, September 2000. 
[TZ99] R. S. Tucker and W. D. Zhong. Photonic Packet Switching. IEICE Trans. Commun., E82-B(2):254-264, February 1999.

[VKM $\left.{ }^{+} 01\right]$ M. Veeraghavan, R. Karri, T. Moors, M. Karol and R. Grobler. Architectures and Protocols that Enable New Applications on Optical Networks. IEEE Communications Magazine, 39(3):118-127, March 2001.

[WCS00] Y. Wang, W. A. Crossland and R. W. Scarr. Modelling for Optically Interconnected Packet Switches. Proc. SPIE 4213, pages 44-55, 2000.

[XPR01] L. Xu, H. Perros and G. Rouskas. Techniques for Optical Packet Switching and Optical Burst Switching. IEEE Communications Magazine, 39(1):136-142, January 2001.

[YBHJ01] Q. Yang, K. Bergman, G. D. Hughes and F. G. Johnson. WDM Packet Routing for High-Capacity Data Networks. Journal of Ligthwave Technology, 19(10):1420-1426, October 2001.

[YJQ97] M. Yoo, M. Jeong and C. Qiao. A High Speed Protocol for Bursty Traffic in Optical Networks. In SPIE Proceedings, All Optical Communication Systems: Architecture, Control and Network Issues, 3230:79-90, November 1997.

[YMD00a] S. Yao, B. Mukherjee and S. Dixit. Advances in Photonic Packet Switching: an Overview. IEEE Communications Magazine, 38(2):84-94, February 2000.

[YMD00b] S. Yao, B. Mukherjee and S. Dixit. Asynchronous Optical PacketSwitched Networks: a Preliminary Study of Contention Resolutions, 2000 .

[YQ98] M. Yoo and C. Qiao. A New Optical Burst Switching Protocol for Supporting Quality of Service. In SPIE Proceedings, All Optical Networking: Architecture, Control and Management Issues, 3531:396405, November 1998.

[YX97a] M. Yoo and C. Xiao. Just-enougth-time(JET): A High Speed Protocol for Bursty Traffic in Optical Networks. IEEE/LEOS Technologies for Global Information Infrastructure, August 1997.

[YX97b] M. Yoo and C. Xiao. Optical Burst Switching (OBS) - A New Paradigm for an Optical Internet. Journal of High Speed Network, 8(1), August 1997.

[YYMD00] S. Yao, S. J. B. Yoo, B. Mukherjee and S. Dixit. All-Optical PacketSwitched Networks: A Study of Contention-resolution Schemes in an Irregular Mesh Network with Variable-Sized Packets. OPTICOMM 2000, October 2000. 
[ZJM00] H. Zang, J. P. Jue and B. Mukherjee. Capacity Allocation and Contention Resolution in a Photonic Slot Routing All-Optical WDM Mesh Network. Journal of Ligthwave Technology, 18(12):1728-1741, December 2000. 